\title{
Neural correlates of conscious visual processing
}

\author{
Dissertation \\ for the award of the degree \\ "Doctor rerum naturalium" \\ of the Georg-August-Universität Göttingen
}

within the doctoral program

Göttingen Graduate School for Neurosciences, Biophysics, and Molecular Biosciences (GGNB) Systems Neuroscience

of the Georg-August University School of Science (GAUSS)

submitted by

Eva Poland

from Nürnberg

Göttingen, 2020 
Prof. Dr. Melanie Wilke

Department of Cognitive Neurology

University Medical Center Göttingen (UMG)

Prof. Dr. Stefan Treue

Department of Cognitive Neuroscience

German Primate Center (DPZ)

Prof. Dr. Annekathrin Schacht

Department of Affective Neuroscience and Psychophysiology

Georg-Elias Müller Institute of Psychology

\section{Members of the Examination Board}

Reviewer: Prof. Dr. Melanie Wilke

Department of Cognitive Neurology

University Medical Center Göttingen (UMG)

Second Reviewer: Prof. Dr. Stefan Treue

Department of Cognitive Neuroscience

German Primate Center (DPZ)

Further members of the Examination Board:

Prof. Dr. Annekathrin Schacht

Department of Affective Neuroscience and Psychophysiology

Georg-Elias Müller Institute of Psychology

PD Dr. Peter Dechent

Department of Cognitive Neurology

University Medical Center Göttingen (UMG)

MD Dr. Arezoo Pooresmaeili

Department of Perception and Cognition

European Neuroscience Institute (ENI)

Prof. Dr. Hansjörg Scherberger

Neurobiology Laboratory

German Primate Center (DPZ)

Date of the oral examination: 15.01 .2021 


\section{Table of contents}

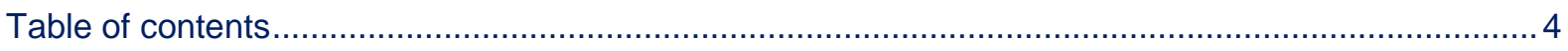

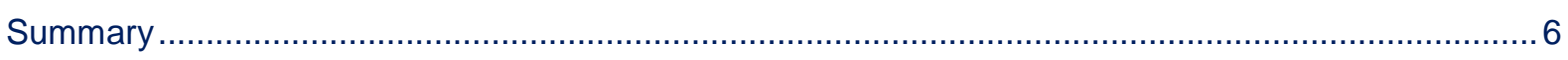

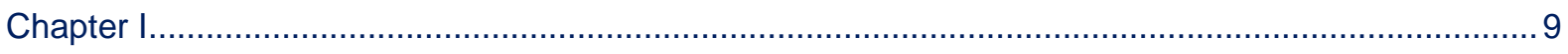

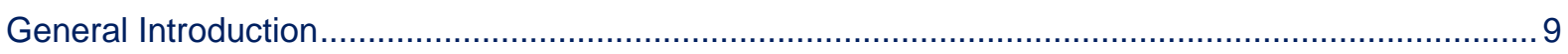

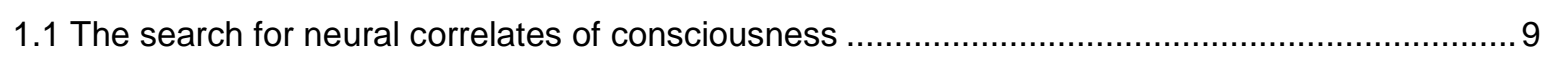

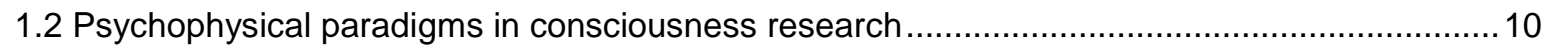

1.2.1 Visual paradigms used to study awareness.......................................................... 10

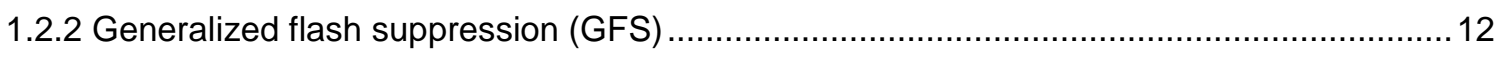

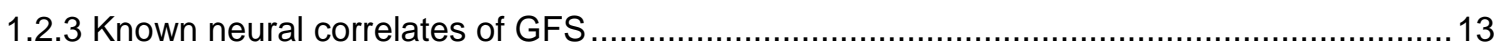

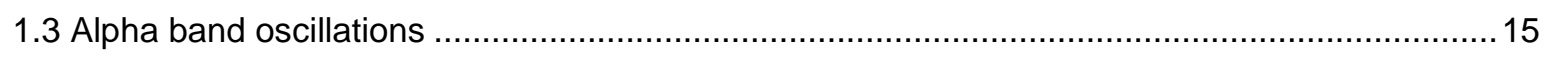

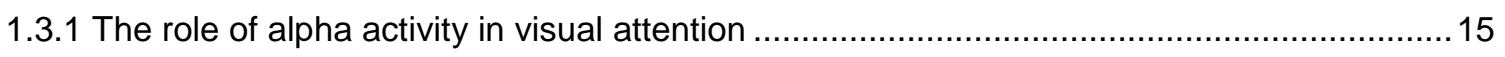

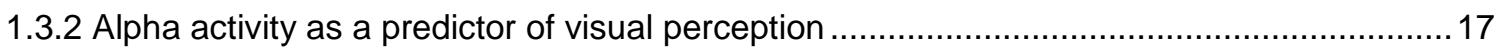

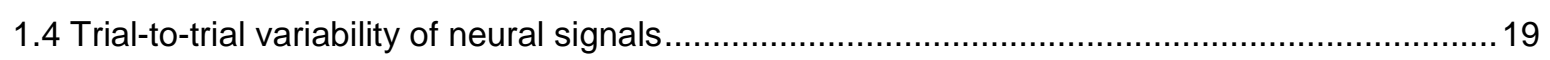

1.4.1 Sources and stimulus-induced changes of neural variability ..................................... 19

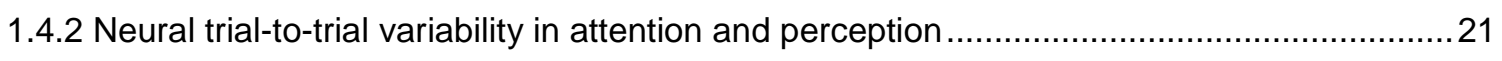

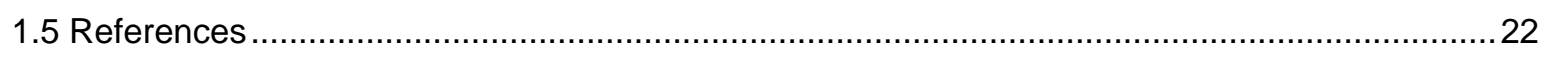

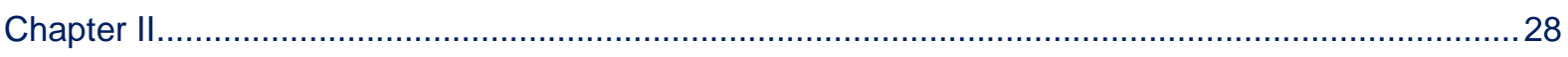

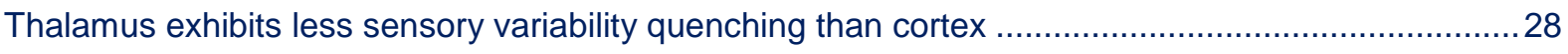

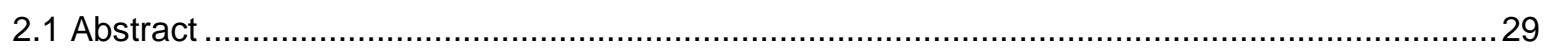

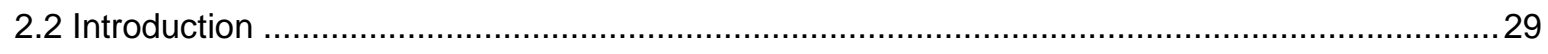

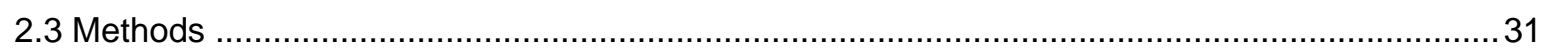

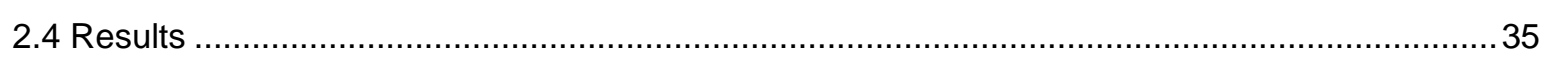

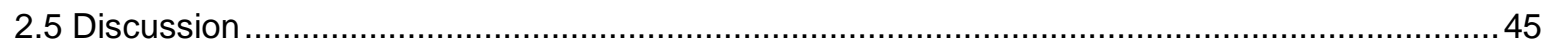

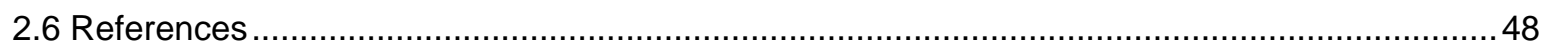

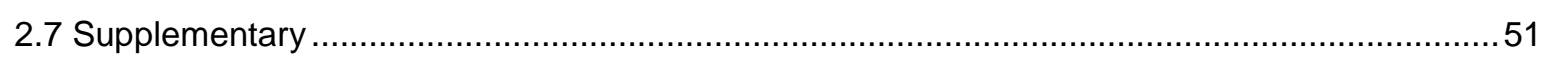

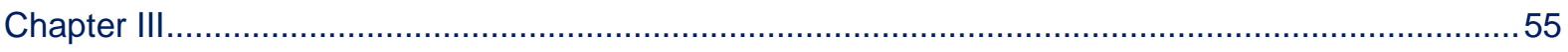

Trial-to-trial variability as a signature of conscious perception ................................................. 55

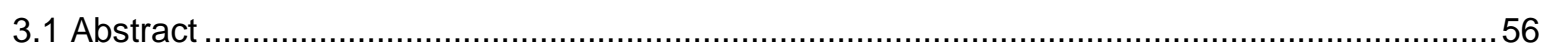

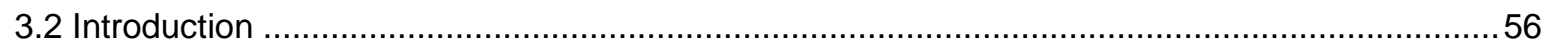

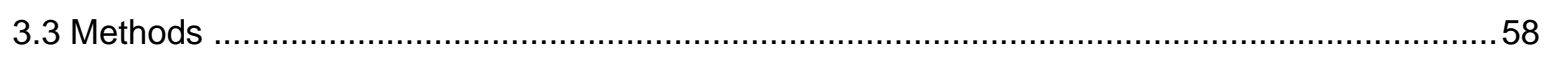

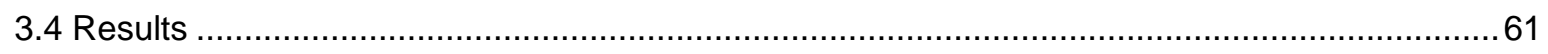

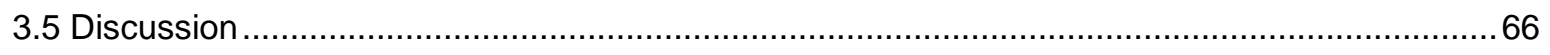

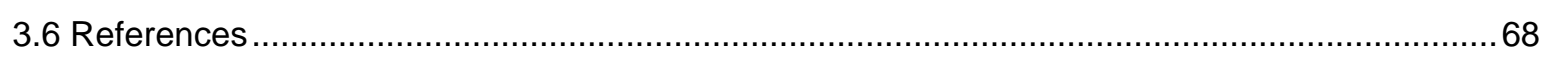

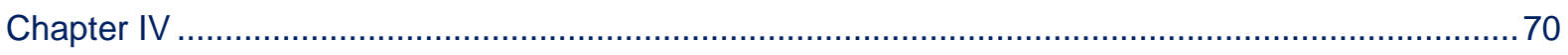

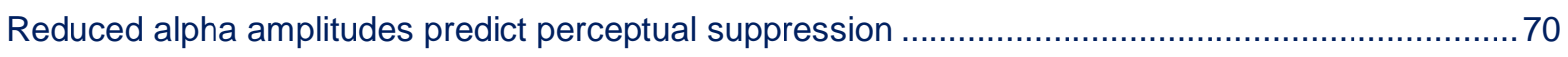

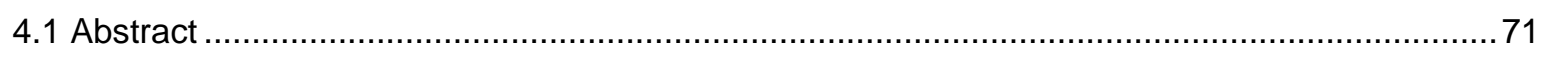

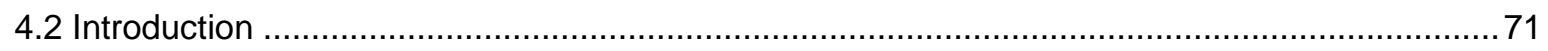




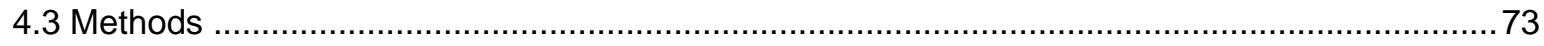

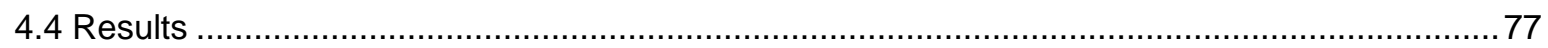

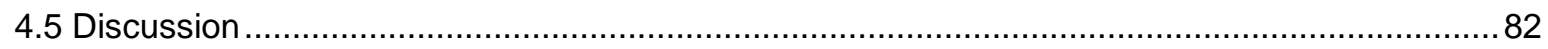

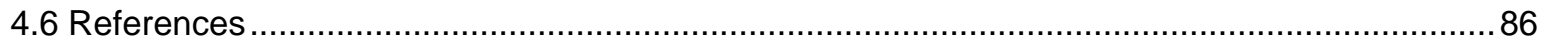

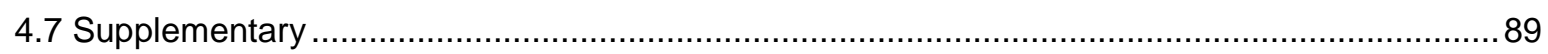

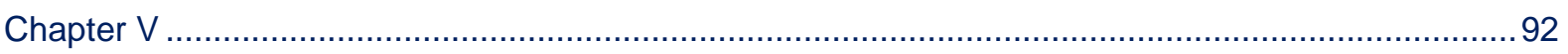

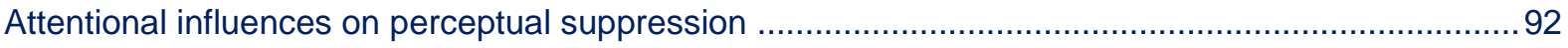

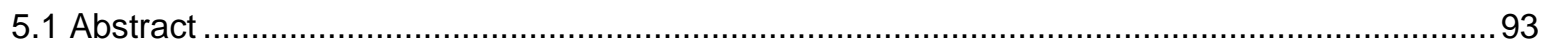

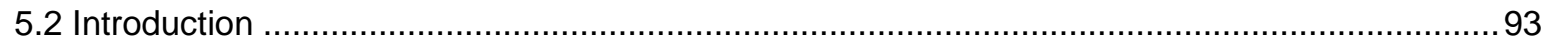

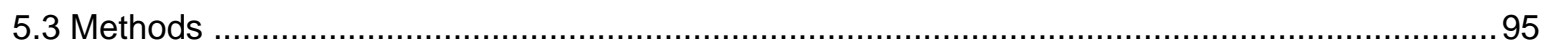

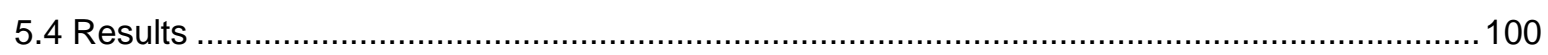

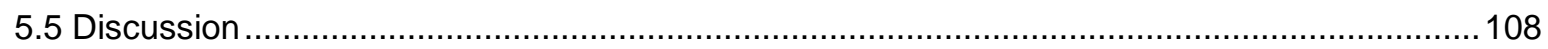

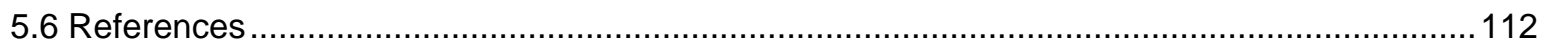

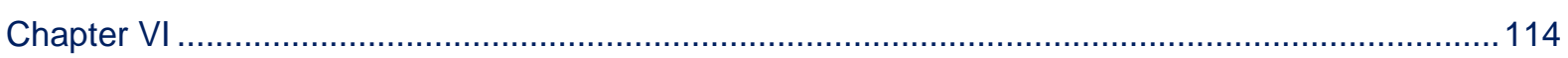

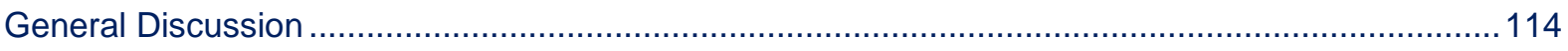

6.1 Neural mechanisms of generalized flash suppression (GFS) ........................................... 114

6.1.1 Conceptual background and summary of the present findings........................................ 114

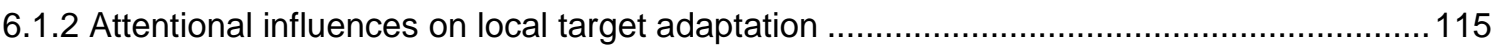

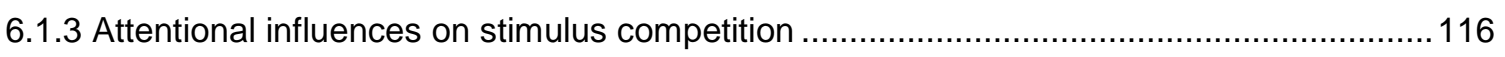

6.1.4 Modulations of neural activity during perceptual suppression ......................................117

6.1.5 Modulations of neural activity predicting perceptual suppression.................................... 118

6.2 Alpha oscillations and neuronal variability in visual awareness .............................................. 120

6.2.1 Conceptual background and summary of the present findings...................................... 120

6.2.2 On the relationship between alpha activity and neural variability ................................... 121

6.2.3 Are stimulus-induced changes determined by ongoing activity? ................................... 123

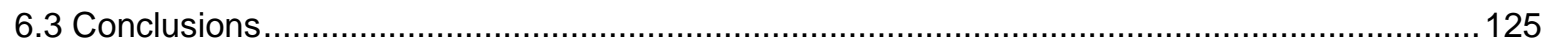

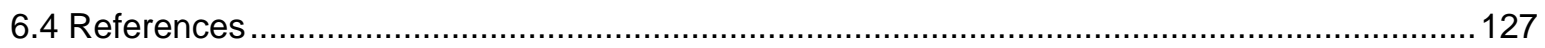

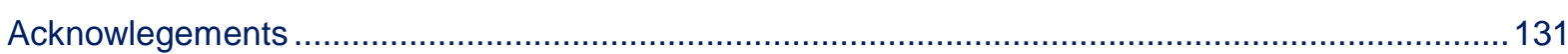

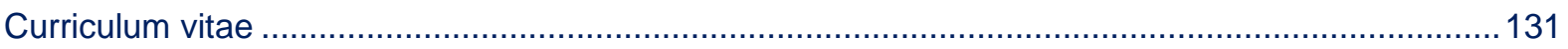




\section{Summary}

The objective of the current thesis is to evaluate the role of alpha band activity and neural trial-to-trial variability in conscious visual perception as well as their relationship to each other. We investigate these measures in electrophysiological recordings of monkeys as well as the electroencephalogram (EEG) of humans using a generalized flash suppression (GFS) paradigm. During GFS, a salient visual stimulus presented to one eye is rendered subjectively invisible on a subset of trials following the presentation of a surrounding motion stimulus to the other eye (Wilke et al., 2003), thereby allowing for the comparison of neural activity corresponding to different states of visual awareness despite identical physical stimulation conditions. A general introduction to these topics is provided in Chapter I.

In Chapter II (Poland et al., 2019), we investigate differences in neural trial-to-trial variability dynamics between thalamus and cortex. To this end we compared single- (SUA) and multi-unit activity (MUA) recorded from extrastriate visual cortex area V4 and sub-regions of the thalamus, the dorsal and ventral pulvinar and the lateral geniculate nucleus (LGN), in two rhesus macaques performing a visual task (Wilke et al., 2009). We show that the decrease in trial-to-trial spiking variability upon stimulus onset previously reported for cortical areas (Churchland et al., 2010) is not present in thalamic regions to the same degree as in visual cortex, and that trial-to-trial variability of the ongoing activity prior to visual stimulation is considerably lower in the thalamus compared to cortex.

In Chapter III, we evaluate neural trial-to-trial variability as a signature of conscious perception in the same V4 data set previously examined in Chapter II, which was originally recorded in the context of a paradigm. We observed higher firing rates and reduced trial-to-trial variability in V4 spiking activity on trials during which the target stimulus was perceptually suppressed. These modulations occurred in a time window $300-800 \mathrm{~ms}$ after the onset of the motion stimulus during the perceptual suppression of the target and resemble modulations previously reported for spatial attention (Cohen and Maunsell, 2009). Examining the corresponding local field potential (LFP) we observed alpha $(8-12 \mathrm{~Hz})$ as well as theta $(4-7 \mathrm{~Hz})$ and beta $(13-20 \mathrm{~Hz})$ amplitudes to be significantly reduced during perceptual suppression, an effect that coincided with the modulations of trial-to-trial variability. We further find V4 alpha amplitude and trial-to-trial variability to be significantly correlated on a site-to-site basis, indicating a possible link between spiking variability and the amplitude of low frequency oscillations. 
In Chapter IV, we investigate modulations of alpha band activity by perceptual suppression and its relationship to neural trial-to-trial variability in a human EEG study in 35 healthy subjects. We modified the original GFS paradigm to present two target stimuli in the left and right visual hemifield in order to evaluate hemisphere-specific effects. Unlike previous studies that reported reduced prestimulus alpha power predicting the visual detection of masked and threshold-level visual stimuli (Ergenoglu et al., 2004; Hanslmayr et al., 2007; Dijk et al., 2008), we find alpha (8-12 Hz) amplitudes of the parietooccipital electrodes spanning visual cortex during the second prior to motion stimulus onset to be significantly lower on trials on which the targets were perceptually suppressed. Moreover, individual prestimulus alpha amplitudes strongly correlate with the decrease in alpha amplitude following stimulus onset as well as with the individual magnitude of the stimulus-induced decrease in trial-to-trial variability, suggesting that both measures are closely linked.

In Chapter V, we directly test the effect of spatial attention on perceptual suppression in a second human EEG study with 30 healthy subjects, hypothesizing that increased attention towards the GFS target stimulus facilitates perceptual suppression. We combined the modified GFS paradigm used in Chapter IV with a visual cueing task prompting subjects to report orientation changes in either the left or right target stimulus during the target adaptation period. We could show that attended targets in both the left and the right visual hemifield were likelier to disappear than unattended targets, suggesting that spatial attention influences the dynamics of perceptual suppression. We find alpha activity to be reduced in the hemisphere contralateral to the attended target, while subsequent target disappearances are in turn predicted by reduced alpha amplitudes in the contralateral hemisphere.

Taken together the studies presented here suggest that neural variability across trials and oscillations in the alpha frequency band are tightly coupled, and that their stimulus-induced changes are dependent on excitability fluctuations in the ongoing activity preceding them. We theorize that these dynamics arise from mechanisms of anticipatory selective attention and propose that the modulations of trial-to-trial variability and alpha amplitudes observed under perceptual suppression do not reflect visual awareness itself but attentional prerequisites for a conscious percept under GFS. We discuss the relationship between alpha activity and neural variability across trials and their role in visual perception as well as the limitations of the present studies in more detail in Chapter VI. 
Churchland MM et al. (2010) Stimulus onset quenches neural variability: a widespread cortical phenomenon. Nat Neurosci 13:369-378.

Cohen MR, Maunsell JHR (2009) Attention improves performance primarily by reducing interneuronal correlations. Nature Neuroscience 12:1594-1600.

Dijk H van, Schoffelen J-M, Oostenveld R, Jensen O (2008) Prestimulus Oscillatory Activity in the Alpha Band Predicts Visual Discrimination Ability. J Neurosci 28:1816-1823.

Ergenoglu T, Demiralp T, Bayraktaroglu Z, Ergen M, Beydagi H, Uresin Y (2004) Alpha rhythm of the EEG modulates visual detection performance in humans. Cognitive Brain Research 20:376-383.

HansImayr S, Aslan A, Staudigl T, Klimesch W, Herrmann CS, Bäuml K-H (2007) Prestimulus oscillations predict visual perception performance between and within subjects. Neurolmage $37: 1465-1473$.

Poland E, Donner TH, Müller K-M, Leopold DA, Wilke M (2019) Thalamus exhibits less sensory variability quenching than cortex. Sci Rep 9:1-12.

Wilke M, Logothetis NK, Leopold DA (2003) Generalized Flash Suppression of Salient Visual Targets. Neuron 39:1043-1052.

Wilke M, Mueller K-M, Leopold DA (2009) Neural activity in the visual thalamus reflects perceptual suppression. PNAS 106:9465-9470. 


\section{Chapter I}

\section{General Introduction}

\subsection{The search for neural correlates of consciousness}

The question which brain mechanisms give rise not merely to our behavior but to our conscious experience of the world is one of the most fascinating problems in neuroscience that remains unresolved to this day. When we attempt to define consciousness, the first distinction that appears necessary is the distinction between the state of conscious and the content of consciousness. We can ask whether a person is in a conscious state, and in the field of medicine different disorders of consciousness such as vegetative state, minimally conscious state, brain death or coma as well as the depth of anesthesia or stages of sleep can be delineated based on their neurological characteristics and the level of responsiveness a patient displays when probed by external stimuli. The second aspect of consciousness then is its content, that is we can ask what a person is aware of at a given moment. In order to investigate the basis of conscious perception, researchers typically employ visual illusions that allow for contrasting neural activity corresponding to one perceptual state with another while sensory information is constant. This focus on a single sensory modality and specific stimuli may be fairly constraint with regard to the complexity of our real world experience, but it allows us to study awareness under controlled laboratory conditions with as much similarity between the compared states as possible. Thus far a number of potential neural correlates of consciousness have been proposed, including neural synchrony particularly in the gamma band, low frequency oscillations, recurrent processing involving several brain areas and recurrent feedback activity between different stages of the sensory processing stream, event-related potential (ERP) components and activations in stimulusspecific higher order sensory cortices as well as in parietal and prefrontal areas (Rees et al., 2002; Lamme, 2006; Koch et al., 2016; Gallotto et al., 2017). In recent years however, doubts have emerged whether these correlates truly reflect visual awareness or other cognitive processes associated with the necessity of subjects reporting about what they perceive, such as attention, working memory, perceptual decision making and motor preparation as well as the behavioral act of reporting itself. These factors may be prerequisites that are necessary for visual awareness to occur or they may be consequences brought about by the awareness of a given stimulus, and their distinct neural 
characteristics may thus differentiate between states of awareness of a stimulus even though they are not a reflection of awareness themselves. The dissociation between awareness and attention poses a particular problem that has sparked an ongoing debate whether both concepts are based on the same neural mechanisms or whether they should be treated as separate phenomena (Lamme, 2003; Koch and Tsuchiya, 2007). The following chapters provide an introduction to psychophysical paradigms to study of visual awareness and two candidate neural signatures of conscious perception that have previously been associated with attention as well as awareness and are the central focus of the present thesis: Neural oscillations in the alpha frequency band and neural variability.

\subsection{Psychophysical paradigms in consciousness research}

Whenever we are presented with visual stimuli specific sets of neurons in our visual cortex will be activated by signals from the retina being transmitted along the pathways of the visual system, and we can infer the presence or absence of a stimulus based on the presence or absence of a neural response. But how then do we infer not the presence or absence of visual stimulation, but whether or not we are consciously aware of a present visual stimulus? A typical approach in consciousness research investigating the neural basis of perceptual awareness is the use of visual illusions by which one state of awareness can be contrasted with another despite physical stimulation conditions being identical in both cases. Employing such illusions thus allows us to disentangle basic requirements of visual perception from subjective awareness. The following sections provide an introduction to commonly used psychophysical paradigms inducing illusory percepts, with a specific focus on the generalized flash suppression (GFS) paradigm employed throughout this thesis. The last section summarizes previously reported neural correlates of GFS.

\subsubsection{Visual paradigms used to study awareness}

One of the earliest and still frequently used techniques to study awareness as well as visual processing in general is visual masking. When a target stimulus is presented very briefly, that is for under around $50 \mathrm{~ms}$, followed by the brief presentation of a masking stimulus, the target stimulus frequently does not reach awareness as visual processing of the target is interrupted by the mask stimulus, a phenomenon 
referred to as backward masking (Kim and Blake, 2005). Backward masking can occur without spatial overlap between the target and the mask stimulus (metacontrast masking), but mask stimuli frequently consist of patterns that are spatially superimposed on the target stimulus (Breitmeyer and Ogmen, 2000). A limitation of visual masking is the requirement for short stimulus presentations, which only allows for the comparison of trials in which the stimulus has either been successfully detected or missed. An alternative paradigm that does not share this temporal constraint and can induce prolonged perceptual suppression of salient visual stimuli is motion-induced blindness (MIB). MIB consists of a dynamic display of one or several static target stimuli with a large moving background. During continuous viewing of the display, the targets spontaneously disappear and reappear, remaining entirely subjectively invisible for several seconds at a time (Bonneh et al., 2001). MIB critically depends on strict fixation and the use of peripheral target stimuli with little complexity, but allows for the comparisons of neural activity during prolonged periods of stimulus invisibility to neural activity during stable perception of the same stimulus (Kim and Blake, 2005).

Another intriguing visual phenomenon that has proven useful in the study of visual awareness is binocular rivalry, during which dissimilar images are presented separately to the left and the right eye. Under these conditions, subjects typically do not perceive both images as superimposed onto each other, but rather their subjective percept alternates between one image and the other (Blake and Logothetis, 2002). As in MIB, the timing of the perception of one image versus another cannot be controlled and reversal rates vary strongly between subjects, but more complex visual stimuli may be studied as any type of visual stimuli sharing the same retinotopic location undergo binocular rivalry during stereoscopic viewing (Kim and Blake, 2005).

Finally, flash suppression paradigms make use of the principle of binocular rivalry, but allow for more control over the timing and the to-be-suppressed stimulus by manipulating relative stimulus onset as well as stimulus features. When an image is first presented to one eye, followed by a second image being abruptly flashed to the other eye, the first image will be perceptually suppressed in favour of the second image (Wolfe, 1984). Other variants of flash suppression include continuous flash suppression (CFS) and generalized flash suppression (GFS). During CFS, a static image is presented to one eye, while the other eye is presented with a stream of rapidly changing patterns. The static image can thereby be suppressed for several seconds without ever being consciously perceived (Tsuchiya and Koch, 2005). During GFS, the static target stimulus is presented for an adaptation period of several hundred 
milliseconds to several seconds prior to the presentation of a suppressing motion stimulus to the other eye, resulting in the timed subjective disappearance of the target with motion stimulus onset (Wilke et al., 2003). By varying stimulus parameters, the probability of perceptual suppression can be adjusted such that the target disappears on approximately half the trials, allowing for the comparison of neural activity between GFS trials on which the suppression was successful and GFS trials in which the target remained visible.

\subsubsection{Generalized flash suppression (GFS)}

Generalized flash suppression (GFS) is a visual illusion during which a salient target stimulus shown to only one eye is rendered subjectively invisible following the presentation of a surrounding motion stimulus to the respective other eye. Phenomenologically, subjects experiencing GFS typically perceive the target stimulus to be absent with only the background visible in its place, thus matching the percept of the eye presented with the motion stimulus. This perceptual suppression of the target occurs in an all-or-none fashion and critically depends on an asynchronous onset of the two competing stimuli, that is several hundreds of milliseconds of adaptation of the target prior to the onset of the moving surround stimulus. Specifically, the disappearance probability of the target stimulus increases with increasing stimulus onset asynchrony (SOA) until saturating at a latency of 1-2 seconds (Wilke et al., 2003). GFS effectively suppresses a wide range of visual stimuli including uniform shapes and Gabor gratings as well as more complex images such as natural scenes and faces (Wilke et al., 2003). A systematic examination of ocular configurations showed that GFS is most effective when the target is only presented to one eye while the motion stimulus is either presented to both eyes or to a different eye than the target stimulus, while target disappearances are considerably less likely during presentations of the target and motion stimulus to the same eye (Wilke et al., 2003). When the target is presented to both eyes and the motion stimulus only to one eye, the percept of the target remains largely stable, indicating that interocular conflict is critical for perceptual suppression under GFS (Wilke et al., 2003). Besides ocular configuration, GFS is affected by a number of stimulus characteristics. GFS has been developed using random dot motion (RDM) for the surrounding motion stimulus and target disappearance probabilities increase with increased dot density as well as a decreased distance between the RDM pattern and the target stimulus, originally referred to as the protection zone (Wilke et al., 2003). Similar to motion-induced blindness (MIB), GFS nonetheless occurs in the absence of local 
conflict between the target and the motion stimulus which do not share retinotopically identical positions (Bonneh et al., 2001; Wilke et al., 2003). GFS is affected by position changes of the target stimulus after the adaptation period with position changes greater than 1 degree of visual angle no longer resulting in subjective target disappearances (Wilke et al., 2003). Changes in target orientation similarly disrupt GFS, while disappearance probabilities are more strongly reduced for orientation changes in a bar stimulus than for orientation changes in a Gabor patch (Wilke et al., 2003).These findings, along with the SOA requirement, strongly suggest a dependence of GFS on visual adaptation of the target stimulus and an involvement of topographical, early visual processing stages (Wilke et al., 2003). Perceptual suppression under GFS thus likely arises from a combination of contributing factors including target adaptation, binocular rivalry and surround inhibition similar to MIB.

\subsubsection{Known neural correlates of GFS}

Three previous studies have investigated the neural basis of GFS. The first study (Wilke et al., 2006) examined multi-unit activity (MUA) as well as the local field potential (LFP) recorded from visual cortex areas $\mathrm{V} 1, \mathrm{~V} 2$ and $\mathrm{V} 4$ of three adult rhesus macaques. In order to present different images to the two eyes, a mirror stereoscope was used. On ambiguous test trials which were used for the analysis, the monkeys were presented with a single salient target stimulus consisting of a red disk or grating. Besides the ambiguous test trials, experimental sessions consisted of 3 to 6 times as many unambiguous control trials in which the ocular configuration was adjusted so that the percept of the target was stable or the target was physically removed from screen in order to ensure the reliability of the monkeys' perceptual reports. The analysis of MUA power following the onset of the surround pattern inducing perceptual suppression on a subset of ambiguous trials revealed that spiking activity in early visual cortex areas V1 and V2 did not reliably reflect the perceptual outcome, whereas MUA in extrastriate visual cortex area V4 was modulated by perceptual suppression. Sites that showed a decrease in response to physical removal of the target and sites that responded with a rate increase were analysed separately. In V4 sites that had shown negative responses to physical removal, MUA activity after surround onset was significantly lower on invisible trials, whereas in sites with positive responses to physical removal, activity was higher when the target was perceptually suppressed. The analysis of the LFP revealed that alpha range $(9-14 \mathrm{~Hz})$ power following surround onset was modulated throughout visual cortex areas, 
showing reduced alpha power on trials in which the target had been reported as invisible, which occurred slightly later than the perceptual modulations of MUA. Modulations of theta, beta and gamma frequency bands were only observed in area V4 and showed the same effect directionality with reduced power during perceptual suppression trials compared to trials on which the target had remained subjectively visible.

The second study (Maier et al., 2008) investigated primary visual cortex activity recorded from two adult rhesus macaques and compared V1 spiking activity, LFP and BOLD responses in the same animals. To accommodate fMRI experiments, the authors employed unambiguous perceptual conditions by manipulating ocular configurations. In the case of invisible trials, the target was presented monocularly while the surround pattern was shown to both eyes, resulting in a high probability of subjective target disappearances. During visible trials, the order of target and surround presentation was either reversed or the target was presented to both eyes, thus increasing the likelihood of the target remaining visible. Comparing these not entirely physically identical but highly similar trials, the authors found the BOLD signal measured in $\mathrm{V} 1$ regions representing the target to reflect target visibility, showing significantly reduced activity during subjective disappearances that was at a level comparable to the physical removal of the target. Interestingly, these differences were not reflected in V1 spiking activity. Consistent with Wilke et al., 2006, an analysis of the LFP showed reduced low-frequency power $(5-30 \mathrm{~Hz})$ during perceptual suppression compared to trials in which the stimuli were adjusted to result in a stable percept of the target, but no modulation of higher frequency bands was observed.

Finally, the third study (Wilke et al., 2009) examined LFP and spiking activity in the visual thalamus of two rhesus macaques, more specifically the lateral geniculate nucleus (LGN) and the dorsal and ventral pulvinar. As in the first study, perceptual modulations were assessed by comparing physically identical, ambiguous test trials that were sorted by the monkeys' perceptual report of subjective disappearances. Spiking activity after surround onset reflected the monkeys' percept in both pulvinar sub-regions, but not in the LGN. As previously observed in visual cortex, pulvinar sites showing positive responses to physical removal of the target exhibited greater activity when the target was perceptually suppressed compared to visible trials while pulvinar sites with rate decreases in response to physical removal showed reduced activity compared to visible trials. Examining the LFP, the authors consistently observed reduced alpha and beta range power during perceptual suppression compared to visible trials in the pulvinar sub-regions, while no perceptual modulation of low-frequency power was observed in 
the LGN. In a control experiment, the perceptual conditions were made unambiguous by either presenting the target and the surround stimulus to the same eye (visible) or to different eyes (invisible) and recording electrophysiological signals while the monkeys were not reporting their percept. Under these passive viewing conditions, pulvinar spiking activity still differentiated between visible and invisible trials, but the modulation of alpha and beta power was no longer observed.

\subsection{Alpha band oscillations}

The sum potential of many cortical neurons recorded through the scalp, the electroencephalogram (EEG), shows oscillatory activity that is thought to regulate functional connectivity between different areas of the brain. Troughs of these brain rhythms constitute periods of minimal or minimally synchronized activity of the neural population from which the signal is recorded, whereas peaks represent periods of high excitability in which a large number of neurons are simultaneously active, thereby facilitating communication within and between neuronal populations that are concurrently in an excitable state. The most prominent rhythm in human cortex are alpha band oscillations occurring at a frequency of around $10 \mathrm{~Hz}$, which were described as early as 1929 by Hans Berger (Berger, 1929). In the first electrophysiological experiments performed on human subjects, Berger observed that the amplitude of the alpha wave was largest when subjects had their eyes closed and were disengaged from their sensory environment, while it characteristically decreased substantially once subjects reopened their eyes (Berger, 1933), a phenomenon now known as event-related desynchronization (ERD) (Pfurtscheller and Lopes da Silva, 1999). With the development of more sophisticated techniques of recording and analyzing oscillatory brain activity, subsequent research has implicated alpha band oscillations to play a significant role in visual perception as well as in higher cognitive functions such as attention and awareness. A summary of these findings is the objective of the following sections.

\subsubsection{The role of alpha activity in visual attention}

Selective attention can be understood as the preferential processing of sensory information that is currently behaviourally relevant, and in principle there are two possible, complementary means to achieve this goal: One, sensory information of a specific category or in a location in space that is 
relevant can be processed more strongly so that its signal is amplified, and two, competing sensory information that is not relevant can be processed less strongly or even suppressed so as to not distract from what is relevant in a given moment. The hypothesis that alpha band activity may be the neural basis underlying these mechanisms of anticipatory attentional selection was initially proposed by Foxe and colleagues, who observed reduced posterior alpha power prior to auditory-visual compound stimuli when their visual features were attended (Foxe et al., 1998). Subsequent studies using cueing paradigms to covertly direct spatial attention to visual stimuli presented in the left and right hemifield consistently found lower parieto-occipital alpha amplitudes over the hemisphere contralateral to the attended stimulus location (Worden et al., 2000; Sauseng et al., 2005; Kelly et al., 2006; Thut, 2006; Mo et al., 2011), and alpha lateralization indices expressing the ratio between left and right-hemispheric alpha power have been shown to reliably reflect the current focus of spatial attention (Thut, 2006). Expanding on these hemifield-specific effects, alpha power has also been found to differentiate between attention directed to stimuli in the upper or lower visual hemifield (Worden et al., 2000). Although the relationship between alpha band activity and spatial attention is more well-studied, there is also evidence to suggest that alpha oscillations play a similar role in feature-based attention: A study using a cueing paradigm to direct subjects attention to different features of a visual stimulus display could demonstrate that when the feature colour was to be attended while the direction of motion was to be ignored, alpha power increased over dorsal regions processing visual motion, while equivalently, alpha increases over ventral regions processing colour were observed when the direction of motion was the task-relevant feature (Snyder and Foxe, 2010). Taken together, these results suggest that higher amplitudes of alpha oscillations in specific brain regions inhibit visual processing, while processing is facilitated under conditions of reduced alpha power. Whether the function of alpha oscillations primarily lies in the signal enhancement of relevant sensory information or in the suppression of competing distractors, which are difficult to distinguish based on relative comparisons of neural activity, has since been the subject of further studies and ongoing debate (Foster and Awh, 2019). Equating the stimulusinduced decrease in alpha amplitude between visual hemifields by presenting bilateral continuously flickering visual stimuli, Kelly and colleagues observed significant increases in alpha power over the hemisphere processing the to be ignored stimulus relative to baseline, implicating alpha activity in distractor suppression (Kelly et al., 2006). A similar conclusion was reached by a study which observed highly spatially selective increases in alpha power over areas processing unattended locations, even in 
the absence of distractor stimuli (Rihs et al., 2007). Besides the visual modality, changes in alpha power have also been shown to track anticipatory attention to auditory stimuli in the respective sensory cortices (Gomez-Ramirez et al., 2011; Mazaheri et al., 2014) and a recent study addressed the question whether modulations of alpha activity reflect the selection of task-relevant target stimuli or the suppression of distractors with a smart experimental design: To be attended auditory target stimuli or to be ignored auditory distractors were fixed in the front, while the location of the respective other (target or distractor) stimulus was varied as coming from the left or the right side of space, allowing Wöstmann and colleagues to evaluate target- and distractor-specific alpha band modulations independently. In the case in which the distractor was presented centrally, alpha power still decreased in the hemisphere contralateral to the target stimulus while it increased ipsilaterally, tracking the location of the target. Interestingly, when the target stimulus was fixed in the front instead, alpha power lateralization indicated the location of the distractor, showing contralateral increases and ipsilateral decreases (Wöstmann et al., 2019), suggesting that alpha power reflects distractor suppression independent of target selection. Conceptually, the role of alpha activity in suppressing task-irrelevant information has been proposed to be implemented by the flexible inhibition of specific nodes in a neural network according to current taskdemands, which would allow for sensory information to be routed more effectively between other nodes within the network, a theory referred to as gating by inhibition (Jensen and Mazaheri, 2010). More generally, alpha power over posterior cortex has also been shown to correlate with fluctuations in subjective attentional state, whereby decreased alpha activity was associated with higher levels of selfreported attentiveness (Macdonald et al., 2011).

\subsubsection{Alpha activity as a predictor of visual perception}

In order for mechanisms of anticipatory selective attention to play a functional role, they can be expected to affect the processing of upcoming stimuli. Indeed it has been shown that prestimulus alpha activity correlates with the amplitude of event-related potential (ERP) components measured after stimulus onset (Brandt and Jansen, 1991; Barry et al., 2000; Ergenoglu et al., 2004) and predicts subsequent perceptual performance (Hanslmayr et al., 2007; Dijk et al., 2008). More specifically, reduced parietooccipital alpha activity was observed prior to visual stimuli that were consciously perceived despite being masked and/or presented at the perceptual threshold when compared to unperceived stimuli 
(Ergenoglu et al., 2004; Hanslmayr et al., 2007; Dijk et al., 2008; Benwell et al., 2017). Besides predicting a given perceptual outcome, lower average prestimulus alpha amplitudes were also observed in individuals who showed a higher performance in detecting masked stimuli compared to individuals who failed to perceive them (Hanslmayr et al., 2007). This relationship between visual awareness and prestimulus alpha activity has been demonstrated to be causal: Rhythmic TMS in the alpha range, but not other frequency bands, impaired visual detection performance (Romei et al., 2010). Combining contrast judgements of a briefly presented stimulus with ratings of subjective awareness, a more recent study found that alpha power prior to stimulus presentation correlated with visual awareness but not objective perceptual performance (Benwell et al., 2017).

The notion that prestimulus alpha activity predicts perceptual acuity has been challenged by a number of studies indicating that reduced alpha amplitudes more generally reflect increased cortical excitability (Foxe and Snyder, 2011). The individual threshold for illusory visual percepts elicited by rhythmic TMS has been shown to correlate with individual posterior alpha power levels (Romei et al., 2008) and a study comparing two different models for the effect of alpha power on signal detection based on EEG data came to the conclusion that reduced alpha activity reflects a more liberal detection criterion, that is a lower threshold for reporting visual stimulus detection, rather than an improved discrimination ability of whether or not a stimulus had been present (lemi et al., 2017). Similarly, posterior prestimulus alpha activity did not correlate with discrimination accuracy in an orientation discrimination task, but was instead strongly inversely related to the subjects' confidence about their decision (Samaha et al., 2017). A study investigating prestimulus alpha activity in the context of the double-flash illusion and the fusion effect, two visual phenomena in which despite identical physical stimulation either one or two stimuli are perceived on a given trial, found that reduced posterior alpha amplitudes predicted the subjective perception of two stimuli, again suggesting a link to increased neural excitability (Lange et al., 2013). Besides their amplitude, the phase of alpha oscillations has been shown to predict visual detection depending on whether brief stimulus presentations coincided with a peak or a trough, whereby visual stimuli falling into the peak of the alpha wave have a higher probability of being detected (Mathewson et al., 2009); suggesting that rapid cyclical changes in cortical excitability mediated by the alpha rhythm may reflect ongoing fluctuations in visual awareness (Mathewson et al., 2011). 


\subsection{Trial-to-trial variability of neural signals}

Neurons generate action potentials (spikes) during spontaneous activity and increase or decrease their firing rate in response to synaptic inputs. One of the simplest models of neuronal spiking is a Poisson process, characterized by individual spikes occurring independently of each other, which results in the variance of spike counts being approximately equal to the average number of spikes. Neural variability is frequently measured as the Fano factor, the ratio between the variance and the mean of spike counts across repeated presentations of the same stimulus. In cortical neurons, the Fano factor is typically greater than one, indicating that cortical responses are highly variable. This additional variance is largely correlated between neurons (Cohen and Kohn, 2011) and substantially decreases with external stimulus input, an effect referred to as variability quenching (Churchland et al., 2010). Commonly accepted models of brain function propose that sensory information is encoded in the neurons firing rate, which can be accurately inferred at the population level (Shadlen and Newsome, 1998), but it is still unclear whether second-order statistics such as neural variability and the exact temporal patterns of spiking activity constitute mere noise or may themselves contain information. The following sections examine neural variability, its potential sources, its stimulus-induced decline and its relation to visual attention and perception in more detail.

\subsubsection{Sources and stimulus-induced changes of neural variability}

Repeated presentations of identical stimuli generate variable numbers of spikes in the same cortical neurons (Tolhurst et al., 1983; Snowden et al., 1992; Britten et al., 1993; Gur et al., 1997) and the time between individual spikes, the inter-spike interval (ISI), is similarly highly variable (Softky and Koch, 1993). Both types of variability appear to be present throughout cortical areas to approximately the same degree, with the variance of spike counts typically around the order of 1.5 times the mean spike count (Lee et al., 1998; Shadlen and Newsome, 1998). This homogeneity does however not seem to generalize to subcortical structures as studies comparing spiking variability in the lateral geniculated nucleus (LGN), a structure of the thalamus transmitting retinal signals to the primary visual cortex $\mathrm{V} 1$, with that in visual cortex areas have consistently found variability in the LGN to be lower (Kara et al., 
2000; Goris et al., 2014; Schölvinck et al., 2015). Spiking variability in cortex has further been shown to be highly correlated between neurons (Cohen and Kohn, 2011; Schölvinck et al., 2015).

What are the sources of neural variability in cortical neurons? Theoretically, there are a number of potential contributing factors, including variable physical stimulus information as well as variability inherited from thalamic inputs, variability in the spike generation of individual neurons, and variability in the ongoing cortical activity that may exert a modulatory influence on neural responses. In vitro experiments have shown that cortical neurons produce highly reliable spike trains (Mainen and Sejnowski, 1995), suggesting that the variability inherent in spike generation contributes only minimally to the variability observed in cortical responses. It is thus likely that synaptic inputs to a given neuron account for a large portion of the observed variability (Shadlen and Newsome, 1998; Carandini, 2004). While variations in physical stimulus input may be considered negligible under highly controlled experimental conditions, it can be argued that the variability in cortical responses stems from its thalamic inputs rather than from the cortical circuitry (Priebe and Ferster, 2012). This hypothesis is supported by experiments by Sadagopan and Ferster who could show that silencing cortical inputs by local inactivation of the surrounding cortex had little effect on the response variability of V1 neurons (Sadagopan and Ferster, 2012). However, a number of studies examining the relationship between ongoing cortical activity and the response variability of individual neurons arrived at the opposite conclusion. Arieli and colleagues showed that the variability of responses in V1 can be attributed to the magnitude of ongoing activity and that single trial responses can be predicted by the summation of the preceding ongoing activity and a deterministic evoked response (Arieli et al., 1996). Similarly, Schölvinck and colleagues who investigated neural variability in LGN and primary visual cortex V1 found that the additional variability observed in cortical responses was predicted by the sum activity of other neurons in the population, suggesting that neural variability reflects global fluctuations of activity affecting the majority of neurons (Schölvinck et al., 2015). The authors further showed that their magnitude is dependent on cortical state with activity fluctuations being largest during synchronized states (Schölvinck et al., 2015). Goris and colleagues demonstrated that neural response variability arises from fluctuations in cortical excitability that are highly correlated between neurons and increase in strength along the visual pathway (Goris et al., 2014).

Trial-to-trial variability in neuronal spiking activity as well as in the membrane potential of single cells has been shown to be dramatically reduced following stimulus presentation compared to the more 
variable spontaneous activity in the absence of driving stimulus input (Churchland et al., 2010). Importantly, physical stimulation thereby strongly reduces correlated variability that is shared between many neurons (Churchland et al., 2010; Oram, 2011). This variability quenching effect can be observed in a wide range of cortical areas and across a wide range of states including anaesthesia, suggesting that the variability decline originates from low-level mechanisms rather than the influence of microsaccades or attention (Churchland et al., 2010). In theoretical work, Deco and Hugues have shown that neural variability quenching can arise from network effects within an attractor network with balanced excitation and inhibition (Deco and Hugues, 2012), suggesting that variability quenching upon stimulus input constitutes a general property of large, recurrent networks, as similarly predicted earlier (Rajan et al., 2010).

\subsubsection{Neural trial-to-trial variability in attention and perception}

The variability of spiking responses can influence how reliably sensory information is encoded by neuronal signals (Zohary et al., 1994; Shadlen et al., 1996; Parker and Newsome, 1998), in particular when it is highly correlated between neurons and may thus not be cancelled out by pooling across the neuronal population. In electrophysiological experiments recording from macaque visual cortex, trialto-trial spiking variability during the sustained response has been shown to be modulated by selective attention, being considerably lower for attended compared to unattended visual stimuli (Mitchell et al., 2007, 2009; Cohen and Maunsell, 2009; Herrero et al., 2013). Attentional modulations of trial-to-trial variability have been linked to a reduction of slow correlated fluctuations in rate and are thought to improve the signal-to-noise ratio of neural signals even more effectively than attention-dependent increases in firing rate (Mitchell et al., 2009). Ni and colleagues further demonstrated that both trial-totrial variability and correlated variability in visual area V4 closely covary with perceptual performance, showing the same relationship for fast changes in performance mediated by attention and for slow changes mediated by perceptual learning over time ( $\mathrm{Ni}$ et al., 2018). Moreover, the variability of visually evoked potentials (VEP) has been shown to correlate with the level of trial-to-trial variability prior to stimulus onset as well as with response times, suggesting that behavioural performance may be dependent on the reliability of ongoing activity (Ledberg et al., 2012). In humans, stimulus-induced decreases in trial-to-trial variability have been observed in the EEG and therein linked to perceptual performance (Arazi et al., 2017a). What is more, the magnitude of variability quenching with stimulus 
onset measured in parieto-occipital electrodes has been shown to be a remarkably consistent characteristic of individual subjects and to predict lower contrast discrimination thresholds in subjects that showed a stronger variability decrease (Arazi et al., 2017b, 2017a). Greater trial-to-trial variability in stimulus responses as well as ongoing activity compared to healthy controls have also been observed in individuals with neurodevelopmental disorders affecting sensory processing, such as attention deficit hyperactivity disorder (ADHD) (Dinstein et al., 2015; Saville et al., 2015; Gonen-Yaacovi et al., 2016) and autism (Milne, 2011; Dinstein et al., 2012, 2015; Haigh et al., 2015). A greater similarity in neural activation patterns over multiple repetitions has further been observed for words and faces that were remembered compared to forgotten stimuli, suggesting a possible link between trial-to-trial variability and episodic memory encoding (Xue et al., 2010). More recently, reductions in EEG trial-to-trial variability in the human visual system have been associated with spatial attention whereby stronger variability quenching was observed in the hemisphere contralateral to the attended stimulus location (Arazi et al., 2019). Stronger quenching of EEG trial-to-trial variability has further been shown to predict the visual detection of threshold-level stimuli compared to stimuli that were not consciously perceived (Schurger et al., 2015) and reduced trial-to-trial variability of $\mathrm{fMRI}$ activation patterns has also been observed for subjectively visible stimuli using dichoptic color masking (Schurger et al., 2010), suggesting that the level of neural variability across trials may be a potential indicator of visual awareness.

\subsection{References}

Arazi A, Censor N, Dinstein I (2017a) Neural Variability Quenching Predicts Individual Perceptual Abilities. The Journal of Neuroscience 37:97-109.

Arazi A, Gonen-Yaacovi G, Dinstein I (2017b) The Magnitude of Trial-By-Trial Neural Variability Is Reproducible over Time and across Tasks in Humans. eNeuro 4 Available at: https://www.ncbi.nlm.nih.gov/pmc/articles/PMC5739532/ [Accessed May 11, 2019].

Arazi A, Yeshurun Y, Dinstein I (2019) Neural Variability Is Quenched by Attention. The Journal of Neuroscience 39:5975-5985.

Arieli A, Sterkin A, Grinvald A, Aertsen A (1996) Dynamics of Ongoing Activity: Explanation of the Large Variability in Evoked Cortical Responses. Science 273:1868-1871.

Barry RJ, Kirkaikul S, Hodder D (2000) EEG alpha activity and the ERP to target stimuli in an auditory oddball paradigm. International Journal of Psychophysiology 39:39-50. 
Benwell CSY, Tagliabue CF, Veniero D, Cecere R, Savazzi S, Thut G (2017) Prestimulus EEG Power Predicts Conscious Awareness But Not Objective Visual Performance. eNeuro 4 Available at: https://www.ncbi.nlm.nih.gov/pmc/articles/PMC5732016/ [Accessed April 29, 2019].

Berger H (1933) Über das Elektrenkephalogramm des Menschen. Archiv f Psychiatrie 98:231-254.

Berger HJC (1929) Über das Elektrenkephalogramm des Menschen. Archiv für Psychiatrie und Nervenkrankheiten Available at: https://www.scienceopen.com/document?vid=b54c402f-0d91-4dcc8555-555889ca6af8 [Accessed April 13, 2020].

Blake R, Logothetis NK (2002) Visual competition. Nat Rev Neurosci 3:13-21.

Bonneh YS, Cooperman A, Sagi D (2001) Motion-induced blindness in normal observers. Nature 411:798-801.

Brandt ME, Jansen BH (1991) The Relationship Between Prestimulus Alpha Amplitude and Visual Evoked Potential Amplitude. International Journal of Neuroscience 61:261-268.

Breitmeyer BG, Ogmen H (2000) Recent models and findings in visual backward masking: A comparison, review, and update. Perception \& Psychophysics 62:1572-1595.

Britten KH, Shadlen MN, Newsome WT, Movshon JA (1993) Responses of neurons in macaque MT to stochastic motion signals. Visual Neuroscience 10:1157-1169.

Carandini M (2004) Amplification of Trial-to-Trial Response Variability by Neurons in Visual Cortex Charles Stevens, ed. PLoS Biology 2:e264.

Churchland MM et al. (2010) Stimulus onset quenches neural variability: a widespread cortical phenomenon. Nat Neurosci 13:369-378.

Cohen MR, Kohn A (2011) Measuring and interpreting neuronal correlations. Nat Neurosci 14:811819.

Cohen MR, Maunsell JHR (2009) Attention improves performance primarily by reducing interneuronal correlations. Nature Neuroscience 12:1594-1600.

Deco G, Hugues E (2012) Neural Network Mechanisms Underlying Stimulus Driven Variability Reduction. PLOS Comput Biol 8:e1002395.

Dijk H van, Schoffelen J-M, Oostenveld R, Jensen O (2008) Prestimulus Oscillatory Activity in the Alpha Band Predicts Visual Discrimination Ability. J Neurosci 28:1816-1823.

Dinstein I, Heeger DJ, Behrmann M (2015) Neural variability: friend or foe? Trends in Cognitive Sciences 19:322-328.

Dinstein I, Heeger DJ, Lorenzi L, Minshew NJ, Malach R, Behrmann M (2012) Unreliable Evoked Responses in Autism. Neuron 75:981-991.

Ergenoglu T, Demiralp T, Bayraktaroglu Z, Ergen M, Beydagi H, Uresin Y (2004) Alpha rhythm of the EEG modulates visual detection performance in humans. Cognitive Brain Research 20:376-383.

Foster JJ, Awh E (2019) The role of alpha oscillations in spatial attention: limited evidence for a suppression account. Current Opinion in Psychology 29:34-40.

Foxe JJ, Simpson GV, Ahlfors SP (1998) Parieto-occipital $\sim 10 \mathrm{~Hz}$ activity reflects anticipatory state of visual attention mechanisms: NeuroReport 9:3929-3933.

Foxe JJ, Snyder AC (2011) The Role of Alpha-Band Brain Oscillations as a Sensory Suppression Mechanism during Selective Attention. Front Psychol 2 Available at:

https://www.frontiersin.org/articles/10.3389/fpsyg.2011.00154/full [Accessed April 29, 2019]. 
Gallotto S, Sack AT, Schuhmann T, de Graaf TA (2017) Oscillatory Correlates of Visual Consciousness. Front Psychol 8 Available at:

https://www.frontiersin.org/articles/10.3389/fpsyg.2017.01147/full [Accessed April 29, 2019].

Gomez-Ramirez M, Kelly SP, Molholm S, Sehatpour P, Schwartz TH, Foxe JJ (2011) Oscillatory Sensory Selection Mechanisms during Intersensory Attention to Rhythmic Auditory and Visual Inputs: A Human Electrocorticographic Investigation. Journal of Neuroscience 31:18556-18567.

Gonen-Yaacovi G, Arazi A, Shahar N, Karmon A, Haar S, Meiran N, Dinstein I (2016) Increased ongoing neural variability in ADHD. Cortex 81:50-63.

Goris RLT, Movshon JA, Simoncelli EP (2014) Partitioning neuronal variability. Nat Neurosci 17:858865.

Gur M, Beylin A, Snodderly DM (1997) Response Variability of Neurons in Primary Visual Cortex (V1) of Alert Monkeys. The Journal of Neuroscience 17:2914-2920.

Haigh SM, Heeger DJ, Dinstein I, Minshew N, Behrmann M (2015) Cortical Variability in the SensoryEvoked Response in Autism. J Autism Dev Disord 45:1176-1190.

HansImayr S, Aslan A, Staudigl T, Klimesch W, Herrmann CS, Bäuml K-H (2007) Prestimulus oscillations predict visual perception performance between and within subjects. Neurolmage 37:1465-1473.

Herrero JL, Gieselmann MA, Sanayei M, Thiele A (2013) Attention-induced variance and noise correlation reduction in macaque $\mathrm{V} 1$ is mediated by NMDA receptors. Neuron 78:729-739.

lemi L, Chaumon M, Crouzet SM, Busch NA (2017) Spontaneous Neural Oscillations Bias Perception by Modulating Baseline Excitability. J Neurosci 37:807-819.

Jensen O, Mazaheri A (2010) Shaping Functional Architecture by Oscillatory Alpha Activity: Gating by Inhibition. Frontiers in Human Neuroscience 4 Available at: http://journal.frontiersin.org/article/10.3389/fnhum.2010.00186/abstract [Accessed July 25, 2019].

Kara P, Reinagel P, Reid RC (2000) Low Response Variability in Simultaneously Recorded Retinal, Thalamic, and Cortical Neurons. Neuron 27:635-646.

Kelly SP, Lalor EC, Reilly RB, Foxe JJ (2006) Increases in Alpha Oscillatory Power Reflect an Active Retinotopic Mechanism for Distracter Suppression During Sustained Visuospatial Attention. Journal of Neurophysiology 95:3844-3851.

Kim C-Y, Blake R (2005) Psychophysical magic: rendering the visible 'invisible.' Trends in Cognitive Sciences 9:381-388.

Koch C, Massimini M, Boly M, Tononi G (2016) Neural correlates of consciousness: progress and problems. Nature Reviews Neuroscience 17:307-321.

Koch C, Tsuchiya N (2007) Attention and consciousness: two distinct brain processes. Trends in Cognitive Sciences 11:16-22.

Lamme VAF (2003) Why visual attention and awareness are different. Trends in Cognitive Sciences 7:12-18.

Lamme VAF (2006) Towards a true neural stance on consciousness. Trends in Cognitive Sciences 10:494-501.

Lange J, Oostenveld R, Fries P (2013) Reduced Occipital Alpha Power Indexes Enhanced Excitability Rather than Improved Visual Perception. J Neurosci 33:3212-3220.

Ledberg A, Montagnini A, Coppola R, Bressler SL (2012) Reduced Variability of Ongoing and Evoked Cortical Activity Leads to Improved Behavioral Performance. PLoS One 7 Available at: https://www.ncbi.nlm.nih.gov/pmc/articles/PMC3427304/ [Accessed March 5, 2020]. 
Lee D, Port NL, Kruse W, Georgopoulos AP (1998) Variability and Correlated Noise in the Discharge of Neurons in Motor and Parietal Areas of the Primate Cortex. The Journal of Neuroscience 18:11611170 .

Macdonald JSP, Mathan S, Yeung N (2011) Trial-by-Trial Variations in Subjective Attentional State are Reflected in Ongoing Prestimulus EEG Alpha Oscillations. Front Psychol 2 Available at: https://www.frontiersin.org/articles/10.3389/fpsyg.2011.00082/full [Accessed April 29, 2019].

Maier A, Wilke M, Aura C, Zhu C, Ye FQ, Leopold DA (2008) Divergence of fMRI and neural signals in V1 during perceptual suppression in the awake monkey. Nat Neurosci 11:1193-1200.

Mainen Z, Sejnowski T (1995) Reliability of spike timing in neocortical neurons. Science 268:15031506.

Mathewson K, Lleras A, Beck D, Fabiani M, Ro T, Gratton G (2011) Pulsed out of awareness: EEG alpha oscillations represent a pulsed-inhibition of ongoing cortical processing. Frontiers in psychology 2 Available at: http://dx.doi.org/10.3389/fpsyg.2011.00099 [Accessed November 28, 2016].

Mathewson KE, Gratton G, Fabiani M, Beck DM, Ro T (2009) To See or Not to See: Prestimulus a Phase Predicts Visual Awareness. J Neurosci 29:2725-2732.

Mazaheri A, van Schouwenburg MR, Dimitrijevic A, Denys D, Cools R, Jensen O (2014) Regionspecific modulations in oscillatory alpha activity serve to facilitate processing in the visual and auditory modalities. Neurolmage 87:356-362.

Milne E (2011) Increased Intra-Participant Variability in Children with Autistic Spectrum Disorders: Evidence from Single-Trial Analysis of Evoked EEG. Front Psychol 2 Available at: https://www.frontiersin.org/articles/10.3389/fpsyg.2011.00051/full [Accessed April 13, 2020].

Mitchell JF, Sundberg KA, Reynolds JH (2007) Differential attention-dependent response modulation across cell classes in macaque visual area V4. Neuron 55:131-141.

Mitchell JF, Sundberg KA, Reynolds JH (2009) Spatial Attention Decorrelates Intrinsic Activity Fluctuations in Macaque Area V4. Neuron 63:879-888.

Mo J, Schroeder CE, Ding M (2011) Attentional Modulation of Alpha Oscillations in Macaque Inferotemporal Cortex. J Neurosci 31:878-882.

Ni AM, Ruff DA, Alberts JJ, Symmonds J, Cohen MR (2018) Learning and attention reveal a general relationship between population activity and behavior. Science 359:463-465.

Oram MW (2011) Visual Stimulation Decorrelates Neuronal Activity. Journal of Neurophysiology 105:942-957.

Parker AJ, Newsome WT (1998) SENSE AND THE SINGLE NEURON: Probing the Physiology of Perception. Annual Review of Neuroscience 21:227-277.

Pfurtscheller G, Lopes da Silva FH (1999) Event-related desynchronization. Handbook of Electroencephalography and Clinical Neurophysiology. Revised Series, Volume 6.

AmsterdamElsevier Science. Available at: https://dare.uva.nl/search?identifier=5dbe2072-6e92-4498b9c2-fb490152715e [Accessed June 17, 2020].

Priebe NJ, Ferster D (2012) Mechanisms of Neuronal Computation in Mammalian Visual Cortex. Neuron 75:194-208.

Rajan K, Abbott LF, Sompolinsky H (2010) Stimulus-dependent suppression of chaos in recurrent neural networks. Phys Rev E 82:011903.

Rees G, Kreiman G, Koch C (2002) Neural correlates of consciousness in humans. Nature Reviews Neuroscience 3:261-270. 
Rihs TA, Michel CM, Thut G (2007) Mechanisms of selective inhibition in visual spatial attention are indexed by $\alpha$-band EEG synchronization. European Journal of Neuroscience 25:603-610.

Romei V, Gross J, Thut G (2010) On the Role of Prestimulus Alpha Rhythms over Occipito-Parietal Areas in Visual Input Regulation: Correlation or Causation? J Neurosci 30:8692-8697.

Romei V, Rihs T, Brodbeck V, Thut G (2008) Resting electroencephalogram alpha-power over posterior sites indexes baseline visual cortex excitability. Neuroreport 19:203-208.

Sadagopan S, Ferster D (2012) Feedforward Origins of Response Variability Underlying Contrast Invariant Orientation Tuning in Cat Visual Cortex. Neuron 74:911-923.

Samaha J, lemi L, Postle BR (2017) Prestimulus alpha-band power biases visual discrimination confidence, but not accuracy. Consciousness and Cognition 54:47-55.

Sauseng P, Klimesch W, Stadler W, Schabus M, Doppelmayr M, Hanslmayr S, Gruber WR, Birbaumer N (2005) A shift of visual spatial attention is selectively associated with human EEG alpha activity. Eur J Neurosci 22:2917-2926.

Saville CWN, Feige B, Kluckert C, Bender S, Biscaldi M, Berger A, Fleischhaker C, Henighausen K, Klein C (2015) Increased reaction time variability in attention-deficit hyperactivity disorder as a response-related phenomenon: evidence from single-trial event-related potentials. Journal of Child Psychology and Psychiatry 56:801-813.

Schölvinck ML, Saleem AB, Benucci A, Harris KD, Carandini M (2015) Cortical State Determines Global Variability and Correlations in Visual Cortex. J Neurosci 35:170-178.

Schurger A, Pereira F, Treisman A, Cohen JD (2010) Reproducibility Distinguishes Conscious from Nonconscious Neural Representations. Science 327:97-99.

Schurger A, Sarigiannidis I, Naccache L, Sitt JD, Dehaene S (2015) Cortical activity is more stable when sensory stimuli are consciously perceived. PNAS 112:E2083-E2092.

Shadlen MN, Britten KH, Newsome WT, Movshon JA (1996) A computational analysis of the relationship between neuronal and behavioral responses to visual motion. J Neurosci 16:1486-1510.

Shadlen MN, Newsome WT (1998) The Variable Discharge of Cortical Neurons: Implications for Connectivity, Computation, and Information Coding. J Neurosci 18:3870-3896.

Snowden RJ, Treue S, Andersen RA (1992) The response of neurons in areas V1 and MT of the alert rhesus monkey to moving random dot patterns. Experimental Brain Research 88:389-400.

Snyder AC, Foxe JJ (2010) Anticipatory attentional suppression of visual features indexed by oscillatory alpha-band power increases: a high-density electrical mapping study. J Neurosci 30:40244032.

Softky WR, Koch C (1993) The highly irregular firing of cortical cells is inconsistent with temporal integration of random EPSPs. J Neurosci 13:334-350.

Thut G (2006) -Band Electroencephalographic Activity over Occipital Cortex Indexes Visuospatial Attention Bias and Predicts Visual Target Detection. Journal of Neuroscience 26:9494-9502.

Tolhurst DJ, Movshon JA, Dean AF (1983) The statistical reliability of signals in single neurons in cat and monkey visual cortex. Vision Research 23:775-785.

Tsuchiya N, Koch C (2005) Continuous flash suppression reduces negative afterimages. Nat Neurosci 8:1096-1101.

Wilke M, Logothetis NK, Leopold DA (2003) Generalized Flash Suppression of Salient Visual Targets. Neuron 39:1043-1052. 
Wilke M, Logothetis NK, Leopold DA (2006) Local field potential reflects perceptual suppression in monkey visual cortex. PNAS 103:17507-17512.

Wilke M, Mueller K-M, Leopold DA (2009) Neural activity in the visual thalamus reflects perceptual suppression. PNAS 106:9465-9470.

Wolfe JM (1984) Reversing ocular dominance and suppression in a single flash. Vision Research 24:471-478.

Worden MS, Foxe JJ, Wang N, Simpson GV (2000) Anticipatory biasing of visuospatial attention indexed by retinotopically specific alpha-band electroencephalography increases over occipital cortex. J Neurosci 20:RC63.

Wöstmann M, Alavash M, Obleser J (2019) Alpha Oscillations in the Human Brain Implement Distractor Suppression Independent of Target Selection. J Neurosci 39:9797-9805.

Xue G, Dong Q, Chen C, Lu Z, Mumford JA, Poldrack RA (2010) Greater Neural Pattern Similarity Across Repetitions Is Associated with Better Memory. Science 330:97-101.

Zohary E, Shadlen MN, Newsome WT (1994) Correlated neuronal discharge rate and its implications for psychophysical performance. Nature 370:140-143. 


\title{
Chapter II
}

\section{Thalamus exhibits less sensory variability quenching than cortex}

\author{
Eva Poland ${ }^{1}$, Tobias H. Donner², Kai-Markus Müller ${ }^{3}$, David A. Leopold ${ }^{4}$, Melanie Wilke ${ }^{1,5,6}$ \\ ${ }^{1}$ Department of Cognitive Neurology, UMG, University Medicine Goettingen, Robert-Koch-Str. 40, Goettingen, 37075, Germany \\ ${ }^{2}$ University Medical Center Hamburg-Eppendorf, UKE, Department of Neurophysiology and Pathophysiology, Building N43, \\ Martinistr. 52, 20246 Hamburg, Germany \\ ${ }^{3}$ Consumer Behavior, HFU Business School, Jakob-Kienzle-Str. 17, 78054 Villingen-Schwenningen, Germany \\ ${ }^{4}$ Section on Cognitive Neurophysiology and Imaging, Laboratory of Neuropsychology, National Institute of Mental Health, \\ National Institutes of Health, Building 49, Room B2J-45, MSC-4400 49 Convent Dr., Bethesda, MD 20892, USA \\ ${ }^{5}$ DFG Center for Nanoscale Microscopy \& Molecular Physiology of the Brain (CNMPB), Robert-Koch-Str. 40, Göttingen, 37075 , \\ Germany \\ ${ }^{6}$ German Primate Center, DPZ, Leibniz Institute for Primate Research, Kellnerweg 4, Goettingen, 37077, Germany
}

\section{Author contributions}

D. A. Leopold, M. Wilke and E. Poland developed the study concept, M. Wilke and K-M. Müller developed the task and collected the data. M. Wilke and E. Poland analyzed the data. D. A. Leopold, T. H. Donner, E. Poland and M. Wilke interpreted the data and conceptually contributed to the analysis process. M. Wilke and E. Poland drafted the manuscript, and D. A. Leopold and T. H. Donner provided critical revisions to the manuscript. All authors provided critical comments during the manuscript writing process and approved the final version of the manuscript. 


\subsection{Abstract}

Spiking activity exhibits a large degree of variability across identical trials, which has been shown to be significantly reduced by stimulus onset in a wide range of cortical areas. Whether similar dynamics apply to the thalamus and in particular to the pulvinar is largely unknown. Here, we examined electrophysiological recordings from two adult rhesus macaques performing a perceptual task and comparatively investigated trial-to-trial variability in higher-order thalamus (ventral and dorsal pulvinar), the lateral geniculate nucleus (LGN) and visual cortex (area V4) prior to and following the presentation of a visual stimulus. We found spiking variability during stable fixation prior to stimulus onset to be considerably lower in both pulvinar and the LGN as compared to area V4. In contrast to the prominent variability reduction in V4 upon stimulus onset, variability in the thalamic nuclei was largely unaffected by visual stimulation. There was a small but significant variability decrease in the dorsal pulvinar, but not in the ventral portion of the pulvinar, which is closely connected to visual cortices and would thus have been expected to reflect cortical response properties. This dissociation did not stem from differences in response strength or mean firing rates and indicates fundamental differences in variability quenching between thalamus and cortex.

\subsection{Introduction}

Cortical activity is characterized by a large degree of variability (Softky and Koch, 1993; Shadlen and Newsome, 1998; Churchland et al., 2010) that poses challenges for relating changes in neural activity to stimulus conditions and behavioural states (Denève and Machens, 2016). At the same time, neural variability itself is increasingly used to infer neurocomputational principles and to assess neural integrity in patient populations (Renart and Machens, 2014; Dinstein et al., 2015). On the level of neuronal spiking, variability across trials, which is typically measured as the mean-corrected firing rate variance (Fano factor), is thought to arise in large part from widespread fluctuations in cortical excitability (Arieli et al., 1996; Sadagopan and Ferster, 2012; Goris et al., 2014; Schölvinck et al., 2015). It has been wellestablished that the onset of a stimulus results in a reduction of trial-to-trial variability, often referred to as variability quenching, that is not a trivial by-product of changing firing rates and thought to constitute 
a common property of a wide range of cortical areas (Churchland et al., 2010). Variability quenching occurs even among neurons that do not exhibit firing rate changes upon stimulus onset, suggesting that the variability decline does not depend on the response properties of individual neurons (Churchland et al., 2010; Schölvinck et al., 2015). Since extensive variability in neural responses limits the reliability with which information can be encoded, its stimulus-driven reduction can be considered to improve sensory processing (Zohary et al., 1994; Shadlen et al., 1996).

Whether equivalent stimulus-induced decreases of spiking variability exist in the thalamus is still largely unclear. Two major thalamic nuclei are closely related to visual cortex: the lateral geniculate nucleus (LGN) and the pulvinar (Saalmann and Kastner, 2011). The LGN is considered a 'first-order' nucleus as it receives driving input from the retina and projects to layer 4 of cortical area V1 (Guillery and Sherman, 2002). The pulvinar is considered a 'higher-order' nucleus which receives major driving input from layer 5 of cortex and participates in cortico-thalamo-cortical pathways (Halassa and Kastner, 2017; Sherman, 2017). While the retinotopically organized ventral pulvinar portion receives input from the superior colliculus and primarily exchanges connections with striate and extrastriate visual cortices, its non-retinotopic dorsal portion primarily interconnects with 'associative' cortices such as superior temporal, posterior parietal, and prefrontal cortices (Gutierrez et al., 2000a; Saalmann and Kastner, 2011; Bickford, 2015; Bridge et al., 2016). Pulvinar neurons in both portions are visually responsive and often modulated by eye movements and internal variables such as visual attention and perception (Benevento and Port, 1995; Berman and Wurtz, 2008; Wilke et al., 2009; Saalmann et al., 2012; Dominguez-Vargas et al., 2017). Both pulvinar portions exchange connections with mid-level visual area V4 (Gattass et al., 2014), and sensory processing in the pulvinar is conceived as a reflection of its cortical inputs as V4 and pulvinar response properties are similar (Zhou et al., 2016). Interactions between the pulvinar and V4 have been shown to be necessary for visual and attentional processing (Saalmann et al., 2012; Zhou et al., 2016). While cortical variability has been extensively studied, few studies have investigated this dimension in the thalamus. Previous studies mainly focused on comparing response variability between visual cortex and first-order sensory thalamus and consistently found LGN responses to be less variable than cortical responses (Kara et al., 2000; Bender and Youakim, 2001; Goris et al., 2014; Schölvinck et al., 2015). Regarding the pulvinar the evidence is less clear: In one study response variability in the pulvinar has been found to be lower than in visual cortices (Bender and Youakim, 2001) while another study in anesthetized ferrets reported higher trial-to-trial 
variability for the lateral-posterior pulvinar complex during visual stimulation compared to V1 (Yu et al., 2016). This discrepancy between the two studies could be attributed to differences in cortical state as higher variability may occur in anesthetized animals when large amplitude, low frequency fluctuations become more prevalent (Schölvinck et al., 2015), but warrants further investigation. Moreover, a systematic investigation of the presence of a quenching effect in the thalamus, and in particular in higher-order thalamic nuclei that exchange recurrent connections with the cerebral cortex such as the pulvinar, is still lacking.

The aim of the present study was to examine whether trial-to-trial variability dynamics in the thalamus are fundamentally similar to those in cortex and show an equivalent reduction upon visual stimulation. To investigate this question we compared spiking variability from neurons in dorsal and ventral pulvinar, LGN and area V4 recorded simultaneously in two macaque monkeys performing the same perceptual task. We found LGN and pulvinar activity to be considerably less variable than V4 activity even prior to stimulus onset, while visual stimulation did not reduce thalamic variability to a similar extent as in cortex.

\subsection{Methods}

Part of the data from pulvinar and LGN contain neurons and multi-unit data that have been included in a previous paper that focused on average firing rates during perceptual suppression (Wilke et al., 2009). Part of the V4 data set has been included in a paper on the features of visual adaptation in area V4 (Müller et al., 2009). The previous studies did not investigate neural variability. All experiments were approved by and conducted following the guidelines of the National Institutes of Health (Bethesda MD, USA).

\section{Electrophysiological recordings}

We recorded multi-unit (MUA) and single-unit (SUA) activity from ventral area V4 (81 MUA, 19 SUA), the thalamic pulvinar (dorsal pulvinar: 102 MUA, 34 SUA; ventral pulvinar: 108 MUA, 12 SUA) and the LGN (89 MUA, 16 SUA) in two adult Rhesus macaques (Macaca mulatta, monkey E and B). Data were recorded during a total of 113 recording sessions (62 monkey E, 51 monkey B) with 4 to 8 microelectrodes simultaneously (Thomas Recording GmbH, Giessen, Germany). Of those sessions, 95 
recordings (49 monkey E, 46 monkey B) contained sites that met our inclusion criteria (uninterrupted recordings without drifts, a minimum number of 10 identical trials as well as a minimum mean firing rate of 5 spikes/s) and were considered for analysis. Spiking activity was collected with the MAP recording system (Plexon Inc., Dallas TX, USA). Spike candidates were identified online through RASPUTIN software (Plexon Inc., Dallas TX, USA) on a PC receiving the digitized signals. Waveform thresholds were manually adjusted for each channel before the start of data acquisition. After the experiment, units were isolated in PCA space in the commercially available "Offline Sorter" (Plexon Inc., Dallas TX, USA) and time stamps were saved for further analysis.

\section{Reconstruction of recording sites}

Surgical procedures, methods and details of reconstruction of the thalamic sites are described in (Wilke et al., 2009) and for V4 in (Müller et al., 2009). In short, recording sites were identified on the basis of chamber coordinates, using MRI scans with gadolinium filled chamber grids. For the thalamic recordings, LGN was used as an additional reference point, which was identified on the basis of its monocular responses in combination with small receptive field sizes. There are multiple parcellation schemes available for the pulvinar (Stepniewska, 2004; Jones, 2012). In the absence of histology but equipped with high resolution 4.7T MRI, we coarsely separated the pulvinar into a ventral and a dorsal portion, using the brachium of superior colliculus as division line, which is well visible on the MRI scans and has been used in previous studies (Gutierrez et al., 2000b; Wilke et al., 2010; Komura et al., 2013; Dominguez-Vargas et al., 2017). The dorsal pulvinar (dPul) includes the medial pulvinar and the dorsal part of the lateral pulvinar (also denoted as PLdm, or Pdm in earlier papers (e.g. (Robinson and Petersen, 1992)), whereas the ventral pulvinar contains the inferior pulvinar and ventral part of lateral pulvinar (also denoted as PLvl) (Robinson et al., 1986; Kaas and Lyon, 2007). The majority of the reported dorsal pulvinar sites were recorded from the dorsal part of the lateral pulvinar (Wilke et al., 2009).

\section{Stimuli and task}

Electrophysiological data were collected in the context of a Generalized Flash Suppression (GFS) paradigm (Wilke et al., 2009). Stimuli were displayed on $38 \times 65 \mathrm{~cm}$ monitors through a mirror 
stereoscope. The screen to eye distance was $88 \mathrm{~cm}$. A small fixation spot $\left(0.15^{\circ}\right)$ was always presented in the middle of the screen and monkeys were required to maintain fixation within a radius of $0.7^{\circ}$ visual angle. Monkeys were required to fixate for $1500 \mathrm{~ms}$ before the target stimulus appeared at a parafoveal position. Target stimuli consisted either of a red luminance patch or grating of $0.3^{\circ}$ to $6^{\circ}$ visual angle and the eccentricity of the targets $\left(0.3^{\circ}-7.7^{\circ}\right)$ was varied on a session basis depending on the position of the receptive fields (RF) of the best isolated recorded neurons. Following target onset, monkeys had to pull a lever.

Data analysis

Neurophysiological data were processed and analysed offline using custom-written software in MATLAB 2015b (The MathWorks Inc., Natick MA, USA). Data from the two animals were similar (Supplementary Information Figure S1) and are thus considered together. The Fano factor was calculated as the variance divided by the mean of spike counts for each recording site and interval of interest. For graphical representation of trial-to-trial variability over time the Fano factor was computed using a $50 \mathrm{~ms}$ sliding window moving in $10 \mathrm{~ms}$ steps and then smoothed using a moving average. For statistical analysis, the Fano factor was calculated in non-overlapping $50 \mathrm{~ms}$ windows covering the respective trial interval and averaged to obtain a single value per interval. For the analysis of stimulusdriven variability changes we considered target stimuli that were presented to the left eye, which was the ocular configuration that yielded the greatest number of identical trials. The mean number of trials in a given session for this condition was 93 for V4, 82 for ventral pulvinar, 82 for dorsal pulvinar and 85 for LGN sites. We found both V4 and thalamic responses to be well captured by a $300 \mathrm{~ms}$ window following stimulus onset. We compared Fano factors of the $300 \mathrm{~ms}$ pre and $300 \mathrm{~ms}$ post target stimulus intervals and calculated percent change scores for the Fano factor decline with stimulus onset according to (FF post - FF pre) / FF pre for each recording site (V4 $N=100$, vPul $N=118, \mathrm{dPul} N=136$, LGN $\mathrm{N}$ $=105)$ to quantify the magnitude of quenching. Statistical significance was assessed with Wilcoxon signed-rank tests or Wilcoxon rank-sum tests for comparisons between brain regions due to the fact that raw Fano factor values were not normally distributed (Shapiro-Wilk test, $p>0.05$ ). We used onesample as well as two-sample t-tests for the normally distributed percent change values. We categorically applied a Bonferroni correction for multiple comparisons and used a significance level of 
$0.05 / 4=0.0125$ for one-sample tests within regions and a significance level of $0.05 / 6=0.0083$ for twosample tests comparing effects between regions. Significant responses to the visual target stimuli were determined by Wilcoxon rank-sum tests $(p<0.05)$ using a $100 \mathrm{~ms}$ windows immediately preceding stimulus onset and at a latency between 50 and $150 \mathrm{~ms}$ after stimulus onset. To determine the contribution of rising and falling rates on the variability decline following stimulus onset, we calculated the Pearson's correlation coefficient between changes in Fano factor and the absolute visually evoked response for sites that showed significant responses.

To correct for differences in firing rates we used the distribution-matching procedure developed in a previous study (Churchland et al., 2010). Specifically, we calculated mean spike counts of each site in all 12 non-overlapping $50 \mathrm{~ms}$ windows spanning the $300 \mathrm{~ms}$ pre to $300 \mathrm{~ms}$ post stimulus period for each region separately. We then determined the greatest common firing rate distribution at a bin resolution of 5 spikes/s across all pre- and post-stimulus time points for each region. We randomly selected subpopulations of sites yielding the same bin heights as the determined greatest common firing rate distribution so as to simulate a constant mean firing rate over time. Based on these sites we then calculated the Fano factor for each time window and averaged the results across 1000 repetitions of random selection of suitable data points. We further analysed a trial period of stable fixation without changes in the visual input 1000 - 500 ms prior to target presentation for which we pooled the available trials which were identical across the whole session, thus obtaining a mean number of 178 trials for $\mathrm{V} 4$, 161 for the ventral pulvinar, 164 for the dorsal pulvinar and 170 for the LGN. We calculated the average Fano factor for each recording site (V4 N =100, vPul $N=118, \mathrm{dPul} \mathrm{N}=136, \mathrm{LGN} \mathrm{N}=105)$ as a function of the window size in which spikes were counted (10 - $200 \mathrm{~ms}$ in steps of $10 \mathrm{~ms})$ as well as in nonoverlapping $50 \mathrm{~ms}$ windows for statistical comparison. Finally, we calculated the Pearson's correlation coefficient between the average Fano factor during 1000 - $500 \mathrm{~ms}$ prior to target interval and the subsequent percent change in Fano factor with stimulus onset. 


\subsection{Results}

Spiking variability following visual stimulation

To assess whether the well-established cortical decrease of trial-to-trial variability upon stimulus onset applies to the thalamus, we first examined the effect of visual stimulation on neural trial-to-trial variability of single- and multi-unit activity recorded in area V4 (81 MUA, 19 SUA), the ventral (108 MUA, 12 SUA) and dorsal pulvinar (102 MUA, 34 SUA) and the LGN (89 MUA, 16 SUA) of two rhesus macaques performing a detection task (Figure 1A).
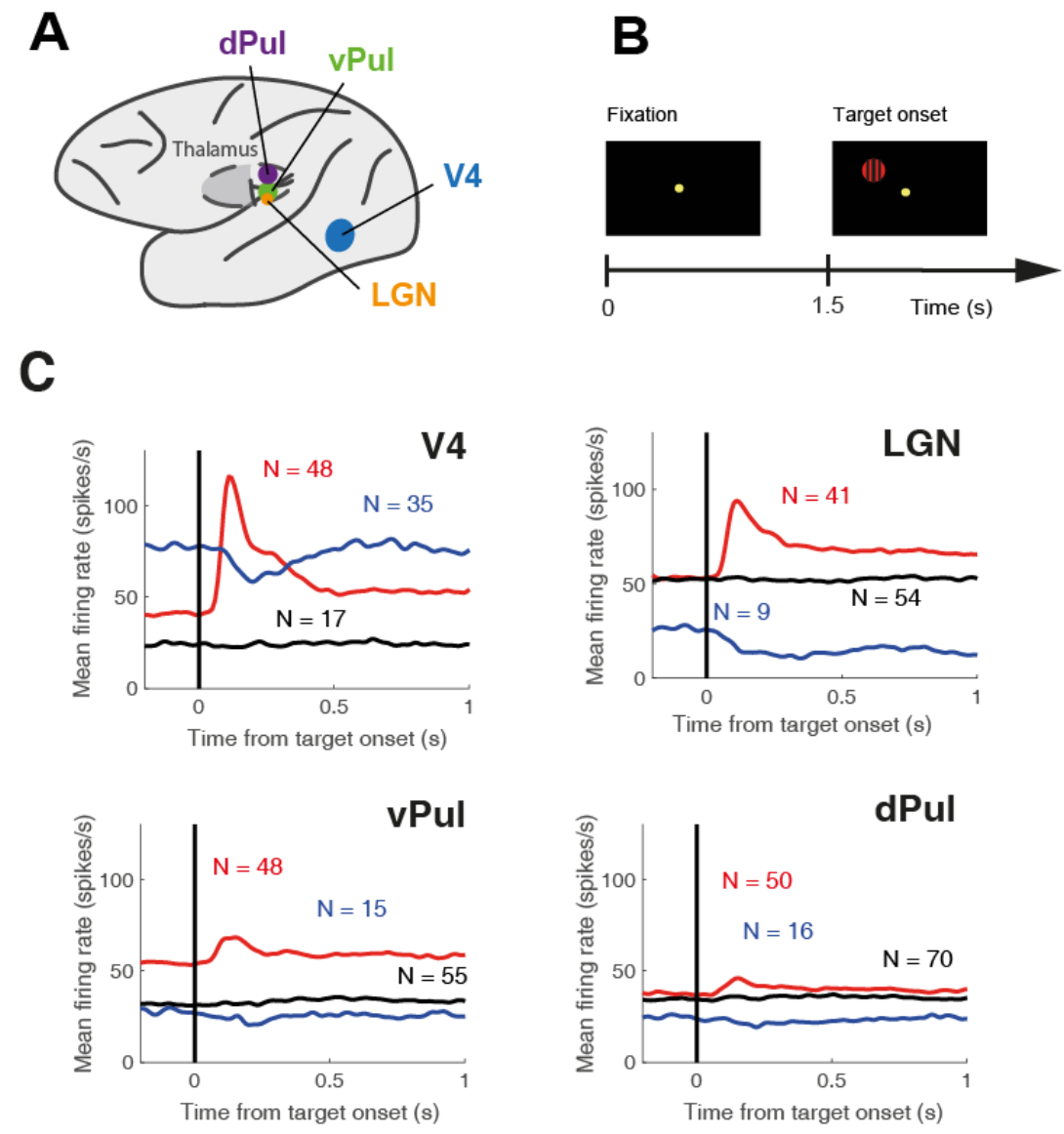

Figure 1. Recording sites and stimuli. (A) Schematic of the recording sites in monkeys $E$ and $B$ : and V4 (blue), ventral pulvinar (vPul, green), dorsal pulvinar (dPul, purple), lateral geniculate nucleus (LGN, orange). MRI-based reconstructions of the thalamic recording sites can be found in ${ }^{24}$. (B) Visual stimuli were presented through a mirror stereoscope. Each trial began with acquisition of the central fixation spot. $1.5 \mathrm{~s}$ later the target stimulus (red disk or grating) was shown. Target stimuli were shown to the left eye. Monkeys were required to pull the lever upon target presentation and to continue holding it as long as the target was visible. Trial intervals of interest were the pre-stimulus fixation period $1000-500$ $\mathrm{ms}$ prior to target onset and visual stimulation $300 \mathrm{~ms}$ pre $-300 \mathrm{~ms}$ post target stimulus. (C) Mean firing rates of sites that showed significant rate increases (red), significant rate decreases (blue) or no significant responses to the onset of the target stimulus (black) for each brain region. 
While the main focus of the current study was trial-to-trial variability in the pulvinar nuclei, we have included the LGN data as a reference point based on previous literature (Kara et al., 2000; Bender and Youakim, 2001; Goris et al., 2014; Schölvinck et al., 2015). The data of the two monkeys were very similar (see Supplementary Information Figure S1 for a separate analysis) and thus pooled. The presentation of the target stimulus (Figure 1B) elicited significant responses in 83 out of 100 sites in area V4, 63 out of 118 sites in the ventral pulvinar, 66 out of 136 sites in the dorsal pulvinar and 50 out of 105 sites in the LGN (Wilcoxon rank-sum test, $p<0.05$, summary Figure $1 \mathbf{C}$ ). Figure $2 \mathrm{~A}$ shows the raw mean spike count and spike count variance across trials $300 \mathrm{~ms}$ prior to (blue) and $300 \mathrm{~ms}$ post target onset (orange) for all sites independent of responsiveness to the visual target. As the Fano factor denotes the variance to mean ratio of spike counts, the slopes of the least squares lines in Figure $2 \mathrm{~A}$ represent trial-to-trial variability within the examined population before (blue) and after stimulus presentation (orange). The estimates based on the slopes of the least-squares lines showed a substantial variability decrease with stimulus onset in area V4, whereas pre and post stimulus slopes in the thalamic pulvinar and LGN sites were similar. The average time course of trial-to-trial variability around the onset of the target stimulus is shown in Figure 2B. As expected from the literature (Mitchell et al., 2007; Cohen and Maunsell, 2009; Churchland et al., 2010), mean trial-to-trial variability in area V4 decreased substantially following onset of the target stimulus. In contrast to cortical activity, trial-totrial variability in the thalamic nuclei was largely unaffected by the visual stimulus presentation. Comparing the average Fano factors of the $300 \mathrm{~ms}$ pre and $300 \mathrm{~ms}$ post target onset intervals in each brain region with Wilcoxon signed-rank tests (Table 1), we observed the expected significant decrease in Fano factor following stimulus onset in area V4 (mean change $-16 \%, p=6.91 \mathrm{e}-11$ ). Spiking variability was unaffected in the ventral pulvinar (mean change $0 \%, p=0.29$ ) and LGN (mean change $2 \%, p=$ 0.55 ) and only marginally, albeit statistically significantly reduced in the dorsal pulvinar (mean change $-4 \%, p=1.72 e-05)$. Figure $2 \mathrm{C}$ shows the distribution of percent change in Fano factor from pre to post stimulus intervals for all recorded sites. While the V4 population shows a clear leftward shift indicating decreased variability following target onset in the majority of individual sites, this shift appears absent in ventral pulvinar and LGN populations and is far less obvious in the dorsal pulvinar. Accordingly, the percent change in Fano factor was significantly greater in area V4 than in ventral and dorsal pulvinar and the LGN (Two-sample t-tests, V4-vPul $p=1.25 \mathrm{e}-12$, V4-dPul $p=2.80 \mathrm{e}-08$, V4-LGN $p=7.14 \mathrm{e}-08$ ). 
A
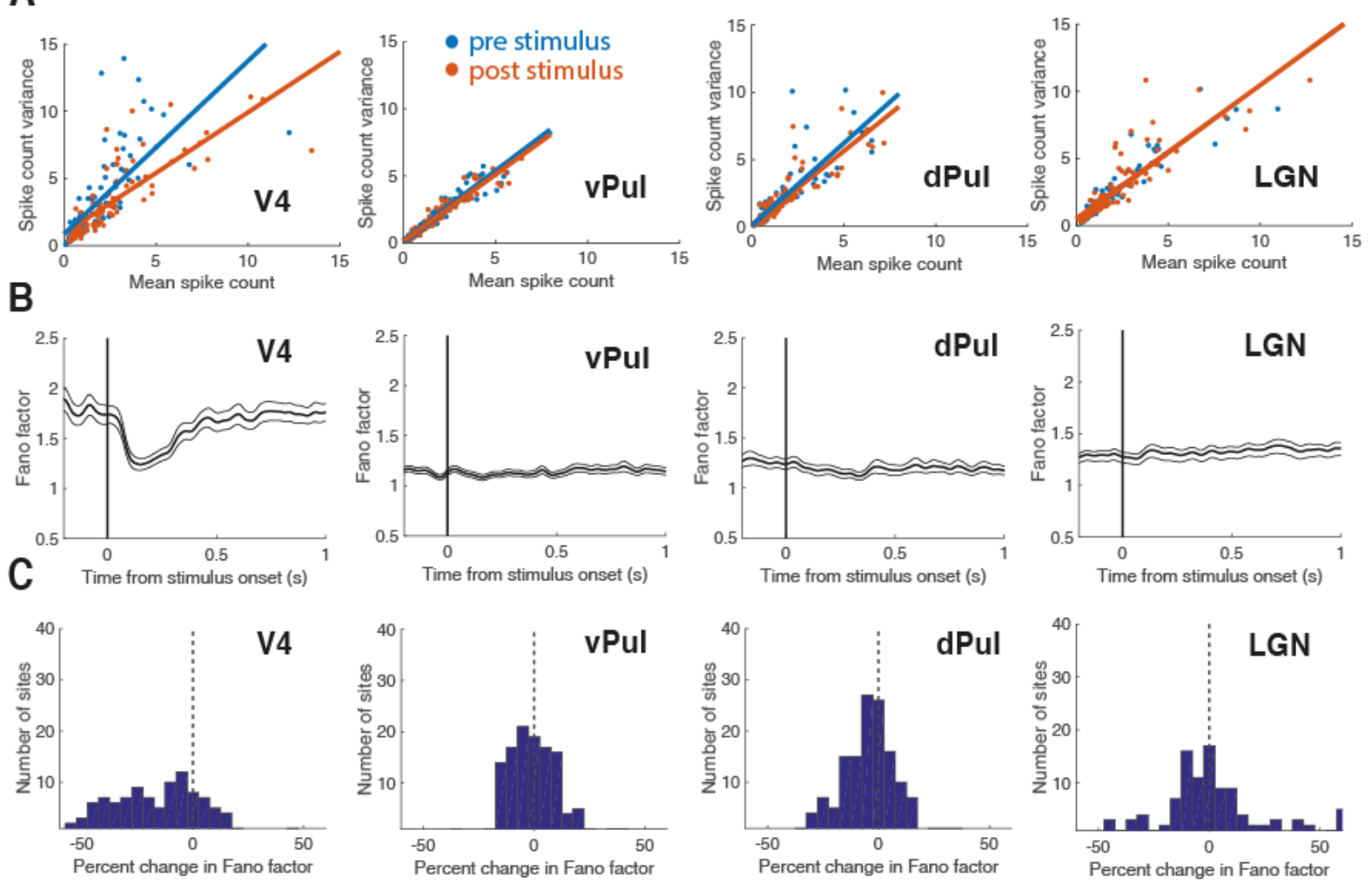

Figure 2. Trial-to-trial variability decrease following visual stimulation. (A) Mean spike count and variance of spike counts during the pre-stimulus window $300 \mathrm{~ms}$ before target onset (blue) and the poststimulus window $300 \mathrm{~ms}$ after target onset (orange) for individual sites regardless of responsiveness (V4 N = 100, vPul $N=118$, dPul $N=136$; $L G N ~ N=105$ ). Lines represent the least squares fit. (B) Average of raw Fano factors aligned to the time of target stimulus onset $(0 \mathrm{~s})$ for complete populations (V4 $\mathrm{N}=100$, vPul $\mathrm{N}=118$, dPul N = 136, LGN N = 105) calculated with a $50 \mathrm{~ms}$ sliding window. Error bars indicate +/- 1 SEM. (C) Population histograms (V4 N = 100, vPul N = 118, dPul N = 136; LGN N = $105)$ of percent change in Fano factor from the pre to post stimulus window.

Response variability, that is variability during the considered post-stimulus window (Table 1), was significantly lower in both pulvinar sub-nuclei than in area V4. Similarly, LGN responses were also less variable than those of V4 but not significantly so (Wilcoxon ranked-sum tests, V4-vPul: $p=9.36 \mathrm{e}-05$, V4-dPul: $p=4.11 \mathrm{e}-05, \mathrm{~V} 4-\mathrm{LGN} p=0.09$ ). A separate examination of SUA and MUA yielded very similar results as we observed a significant quenching effect in both V4 SUA and MUA that was also significantly larger than variability changes in the thalamic regions. The variability decrease in the dorsal pulvinar was only significant in the multi-unit data, which may however be due to the low sample size for single-units (for details see Supplementary Information Figure S2). Since the Fano factor measures the degree of trial-to-trial variability within single neurons or multi-units, it does not by itself provide information as to whether the variability is independent or shared across many neurons. We thus additionally sought to examine correlated variability within and between area V4 and the pulvinar 
regions by calculating the spike count (noise) correlations in simultaneously recorded unit pairs. Calculations of spike count correlations based on multi-unit data can lead to a consistent overestimation of the correlation strength (Cohen and Kohn, 2011), which does however not affect the relative comparison of correlations before and after stimulus onset. Within area V4 shared variability also substantially decreased with the onset of the target stimulus (Supplementary Information Figure S3). We observed no significant change of correlated variability with stimulus onset in the pulvinar, nor between pulvinar and V4.

\begin{tabular}{|c|c|c|c|c|}
\hline & V4 & vPul & dPul & LGN \\
\hline $\mathrm{N}$ & 100 & 118 & 136 & 105 \\
\hline $\begin{array}{c}\text { mean Fano factor } \\
\text { pre stimulus +/- SD }\end{array}$ & $1.80+/-0.98$ & $1.13+/-0.25$ & $1.26+/-0.59$ & $1.28+/-0.45$ \\
\hline $\begin{array}{c}\text { mean Fano factor post } \\
\text { stimulus +/- SD }\end{array}$ & $1.41+/-0.57$ & $1.12+/-0.25$ & $1.19+/-0.51$ & $1.29+/-0.51$ \\
\hline
\end{tabular}

Table 1. Summary of mean Fano factors +/- standard deviation $300 \mathrm{~ms}$ pre and $300 \mathrm{~ms}$ post onset of the target stimulus. $\mathrm{N}$ denotes the number of recording sites.

\section{Relationship to stimulus-induced changes in firing rate}

Given this prominent difference among cortical and thalamic populations with respect to the stimulusinduced variability decline, we next asked whether changes in firing rate might impact variability in cortical and thalamic neurons differently. We examined whether the observed quenching differences between V4 and the thalamic sites could be attributed to differences in the strength of the visually evoked responses. As the pulvinar populations in our data set had a larger percentage of nonresponsive sites and responses were generally less pronounced than in area V4 (Figure 1C), we wondered whether the minimal stabilization effects in the thalamic regions were due to differences in response properties of the examined samples. We thus examined the time courses of trial-to-trial variability around the time of target stimulus onset for the subset of sites which showed positive significant visually evoked responses ( $\mathrm{V} 4 \mathrm{~N}=48$, vPul $\mathrm{N}=48, \mathrm{dPul} N=50, \mathrm{LGN} \mathrm{N}=41$, Figure 3A) and found them to be very similar to the behaviour of the complete population described above. The percent change distribution from pre to post stimulus interval was significantly different from zero in area V4 and the dorsal pulvinar, but not in the ventral pulvinar portion or in the LGN (One-sample ttests, V4 $p=2.57 e-11$, vPul $p=0.02, d P u l p=3.05 e-06$, LGN $p=0.32$ ). 
A
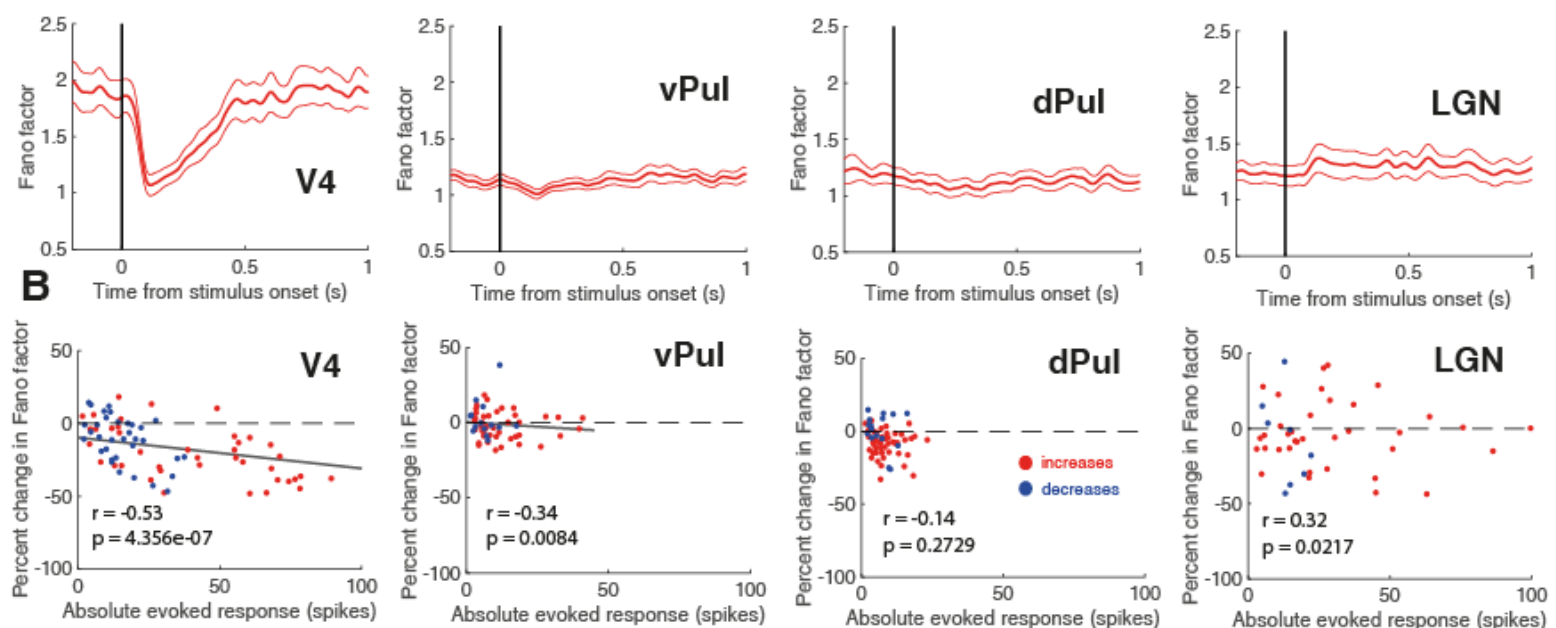

Figure 3. Dependence on the visually evoked response strength. (A) Fano factor around the time of target onset for the subsets of visually responsive sites that responded with significant rate increases (V4 N = 48, vPul N = 48, dPul N = 50, LGN N = 41). Error bars indicate +/- 1 SEM. (B) Percent change in Fano factor from the $300 \mathrm{~ms}$ pre to $300 \mathrm{~ms}$ post stimulus interval as a function of the evoked response strength (absolute spike difference) for sites with significant rate increases (red) and decreases (blue).

Figure 3B shows the percent change in Fano factor as a function of the absolute evoked response strength for individual visually responsive sites. In V4, we found a strong negative correlation between the decrease in Fano factor and the strength of the evoked response (Pearson's correlation coefficient after outlier correction $r=-0.53, p=4.36 e-07)$, suggesting that the variability reduction may have been at least in part due to the rate change itself. This negative correlation was present in sites that responded with rate increases $(\mathrm{N}=48, r=-0.53, p=9.45 \mathrm{e}-05$, red) as well as in sites that responded with a rate decrease $(\mathrm{N}=35, r=-0.54, p=0.10 \mathrm{e}-2$, blue $)$. In the ventral pulvinar we observed a significant negative correlation between the variability decline and the visually evoked response strength $(r=-0.34, p=$ 0.84e-2), while there was no significant correlation after Bonferroni correction in the dorsal pulvinar $(r=$ $-0.14, p=0.27)$ or in the LGN $(r=0.32, p=0.02)$. Due to the dependence on the visually evoked response we sought to exclude that the observed variability decline was primarily caused by rising and falling rates in response to the target stimulus by matching firing rate distributions over the $300 \mathrm{~ms}$ to $300 \mathrm{~ms}$ post stimulus period equivalent to the approach developed in a previous study (Churchland et al., 2010). The original mean firing rate (black) as well as the matched firing rate (blue) around the time of stimulus onset are shown in Figure 4A. The firing rates obtained through the matching procedure were comparable between regions (24 spikes/s in V4, 20 spikes/s in the ventral pulvinar, 19 spikes/s in the dorsal pulvinar and 23 spikes/s in the LGN). We then performed the equivalent statistical analysis 
on the pre and post target intervals using the distribution-matched values (Figure 4B-C). Despite mean firing rates being kept constant over time, the stimulus-evoked variability decline remained significant in V4 and in the dorsal pulvinar, while again not being significant in the ventral pulvinar or in the LGN (Wilcoxon signed-rank test, V4: $N=43, p=3.06 e-07$, vPul: $N=60, p=0.55$, dPul: $N=79, p=5.33 e-$ $07, \mathrm{LGN}: \mathrm{N}=60, \mathrm{p}=0.08$ ), thus confirming the results obtained in the analysis of the full data.

A
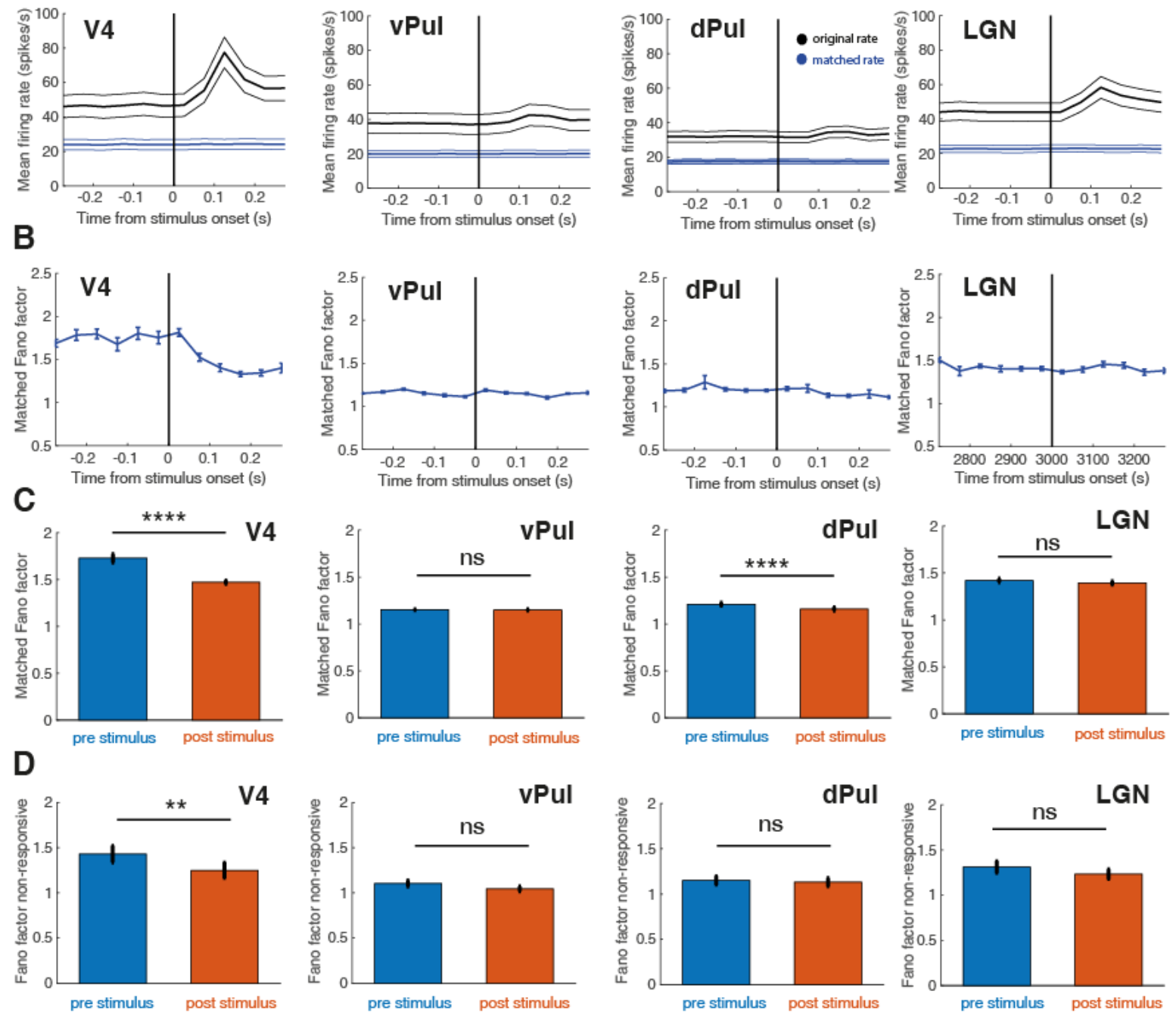

Figure 4. Controls for changes in firing rate. (A) Raw (black, V4 $\mathrm{N}=100$, vPul $\mathrm{N}=118$, dPul $\mathrm{N}=$ 136, $L G N N=105$ ) and distribution-matched mean firing rates (blue, $V 4 N=43, v P u l ~ N=60, d P u l ~ N=$ 79 , $L G N N=60$ ) around the time of stimulus onset. Error bars show +/- 1 SEM. (B) Distribution-matched Fano factor computed from the data points representing the matched rate $(\mathrm{V} 4 \mathrm{~N}=43$, vPul $\mathrm{N}=60$, dPul $\mathrm{N}=79$, LGN N =60). Error bars indicate +/- $1 \mathrm{SEM}$. (C) Mean distribution-matched Fano factor for the $300 \mathrm{~ms}$ pre (blue) and $300 \mathrm{~ms}$ post stimulus interval (orange). Error bars indicate $+/-1 \mathrm{SEM}$. Wilcoxon signed-rank tests, Bonferroni corrected, ${ }^{* * * *} p<0.0001$. (D) Fano factor calculated based on nonresponsive sites (Wilcoxon ranked-sum tests, $p>0.05$; V4 $\mathrm{N}=47$, vPul $\mathrm{N}=79, \mathrm{dPul} N=95, \mathrm{LGN} \mathrm{N}=$ 79) for $300 \mathrm{~ms}$ pre (blue) and $300 \mathrm{~ms}$ post RDM stimulus (orange). Error bars indicate +/- 1 SEM. Wilcoxon signed-rank tests, Bonferroni corrected, ${ }^{* *} \mathrm{p}<0.01$. 
While the Fano factor can be reliably computed based on multi-unit recordings due to the fact that the sum of independent Poisson-distributed values is itself Poisson-distributed, the success of the meanmatching procedure may not be guaranteed (Churchland et al., 2010). As Churchland et al. have previously shown (Churchland et al., 2010), variability decreases with stimulus onset can also be observed in neurons that do not themselves respond to the stimulus. This allowed us to investigate the quenching effect under non-responsive conditions when the firing rate of the examined sites changes little. The target stimulus elicited significant responses in most V4 sites (Figure 1C). We thus examined presentations of a large full-field random dot motion (RDM) pattern, which did not evoke significant visual responses in a large portion of sites (Wilcoxon rank-sum test, $p>0.05$ ). Consistent with previous findings (Churchland et al., 2010) we observed a significant decrease in trial-to-trial variability following the onset of the motion stimulus in area V4 even when the firing rate did not change (Wilcoxon signedrank test, $N=47, p=6.53 e-3$, Figure $4 D$ ). While variability in non-responsive sites decreased slightly throughout all regions, this effect was insignificant in non-responsive ventral pulvinar, dorsal pulvinar or LGN sites (Wilcoxon signed-rank tests, vPul: $N=79, p=0.08$; dPul: $N=95, p=0.48, L G N: N=79, p$ $=0.14)$, further confirming that the differences between thalamic and cortical regions in quenching behaviour did not stem from differences in responsiveness. In addition to changes in firing rate, small eye movements may in principle impact quenching. We thus performed control analyses to account for this possibility and could confirm that the observed differences between regions did not stem from changes in microsaccade rate (Supplementary Information Figures S4).

\section{Variability changes in the dorsal pulvinar}

We were surprised to find a small but significant variability decrease following stimulus onset in the dorsal pulvinar but not in the ventral pulvinar portion, despite the latter being closely interconnected with the visual system and likely receiving inputs from area V4 (Shipp, 2003; Bridge et al., 2016). Since monkeys were required to pull the lever in response to the onset of the target stimulus and variability changes have been related to motor preparation(Churchland et al., 2006), we wondered whether the lever action itself might have contributed to the reduction of the Fano factor in the dorsal pulvinar, where neuronal firing is also related to hand movements (Acuna et al., 1990). We thus undertook a closer inspection of the RDM stimulus, which was not followed by a lever response. This had the additional 
benefit of allowing us to determine whether the different quenching behaviour in cortex and thalamic regions was reproducible with a different stimulus type. As for the target stimulus, we found a substantial decrease in Fano factor following the onset of the RDM stimulus in V4, whereas stimulus-induced changes in trial-to-trial variability in the pulvinar and LGN were again of smaller magnitude. Figure 5AB summarizes the findings for the two stimuli. In contrast to the target stimulus onset with associated lever response (Figure 5A), the full-field motion stimulus did however not elicit a significant variability decrease in the dorsal pulvinar portion (Figure 5B), while area V4 continued to exhibit a significant stimulus-induced variability decline (One-sample t-tests, V4 $p=3.30 \mathrm{e}-09$; vPul $p=0.05 ; \mathrm{dPul} p=0.93$; LGN: $p=0.19)$.

A

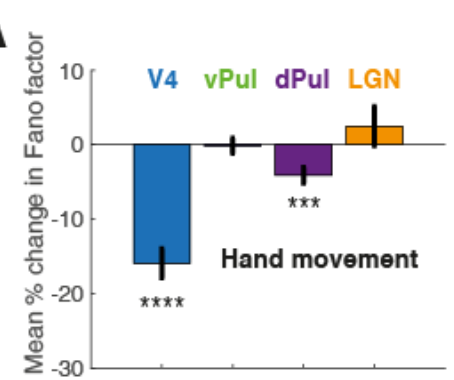

B

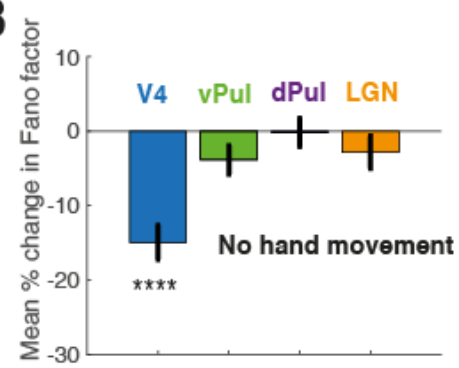

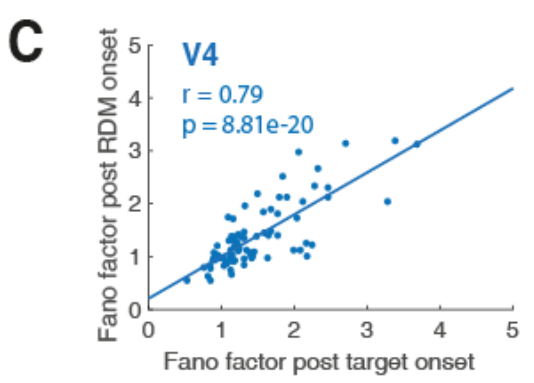

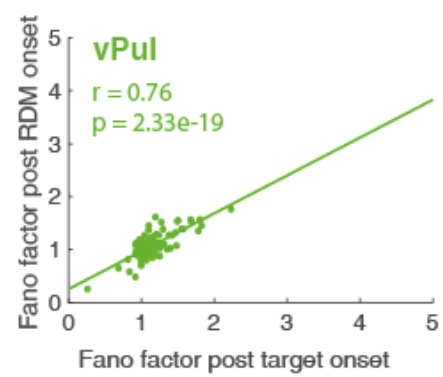

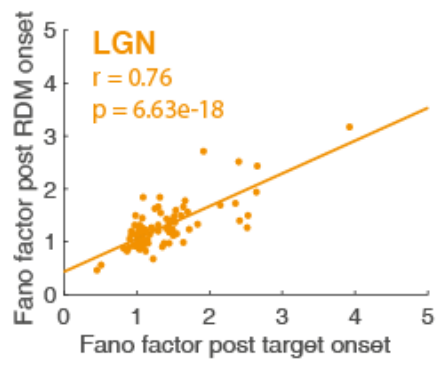

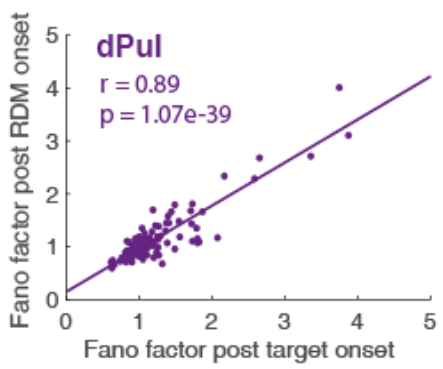

D

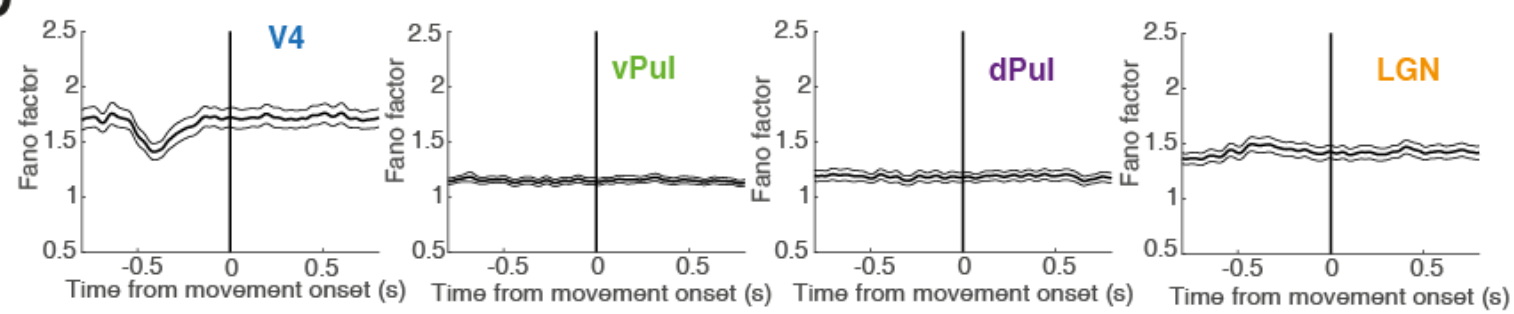

Figure 5. Quenching effect in the dorsal pulvinar and relation to hand movement. (A) Mean percent change in Fano factor from $300 \mathrm{~ms}$ pre to $300 \mathrm{~ms}$ post target stimulus intervals in area V4, the ventral and dorsal pulvinar and the LGN (V4 N = 100, vPul $N=118, \mathrm{dPul} N=136$; $L G N N=105)$. Error bars indicate $+/-1$ SEM. One-sample t-tests, ${ }^{* * * *} p<0.0001,{ }^{* * *} p<0.001$. Upon target presentation, the animal was instructed to pull a lever. (B) Mean percent change in Fano factor from $300 \mathrm{~ms}$ pre to 300 ms post RDM stimulus intervals in area V4, the ventral and dorsal pulvinar and the LGN. Error bars indicate $+/-1$ SEM. One-sample t-tests, ${ }^{* * *} p<0.0001$. The RDM stimulus was not followed by a hand movement. (C) Fano factors for the 300 ms post stimulus interval for the target and the RDM stimulus. Pearson's correlation coefficients. (D) Fano factor time courses of data triggered to the time of the lever pull following the presentation of the target stimulus. 
We compared Fano factor values of the 300 ms post stimulus interval for the target and the RDM stimulus within the common subset of recording sites and found response variability to be highly correlated between stimulus types in all examined regions (Pearson's correlation coefficients, Figure 5C). We thus wondered whether the significant variability decrease in the dorsal pulvinar we previously observed in the presence of a lever response was due to the hand movement and examined trial-totrial variability triggered to the time the animals pulled the lever. 32 out of 136 dorsal pulvinar sites showed significant motor responses (Wilcoxon rank-sum test, $p<0.05$ ). There was no significant difference between Fano factors $300 \mathrm{~ms}$ prior and $300 \mathrm{~ms}$ following the lever response in the dorsal pulvinar (Wilcoxon signed-rank test, $\mathrm{N}=136, \mathrm{p}=0.14$ ) and Fano factor time courses triggered to the lever action were flat (Figure 5D), suggesting that the small but significant variability decline following the target stimulus was not due to the movement itself, but may nonetheless have been related to motor preparation as it was no longer present in the absence of a subsequent motor response.

\section{Spiking variability prior to stimulus onset}

In examining the effect of visual stimulation on trial-to-trial variability, we noted that neural activity was already considerably less variable in the pulvinar and the LGN than in area V4 prior to stimulus onset (Figure 2B). This led us to wonder whether the absence of quenching in the pulvinar and LGN compared to cortex was related to thalamic spiking variability already being relatively low when sites were not directly driven by the onset of a stimulus. We thus examined a $500 \mathrm{~ms}$ period during stable fixation that began $500 \mathrm{~ms}$ after the acquisition of the fixation spot and ended $500 \mathrm{~ms}$ before the onset of the target stimulus (see Figure 1B) in order to determine whether the degree of baseline variability was similar among the different regions. In the absence of changes in visual input, spiking activity was considerably more variable in area V4 than in the thalamic pulvinar and LGN (Figure 6A, Table 2) (Wilcoxon rank-sum tests, Bonferroni corrected, V4-vPul: $p=4.19 e-11$; V4-dPul: $p=7.08 e-11$, V4-LGN: $p=1.00 \mathrm{e}-06$ ). Albeit firing rates tended to be lower in the pulvinar as compared to both V4 and LGN (Figure 6B), the mean firing rates during the examined interval were not statistically different after Bonferroni correction (Wilcoxon rank-sum tests, V4-vPul $p=0.33$; V4-dPul $p=0.03$; V4-LGN $p=0.99$; vPul-LGN $p=0.33$; dPul-LGN $p=0.01$; vPul-dPul $p=0.16$ ). Generally, we found trial-to-trial variability to increase with the size of the counting window. Figure $6 \mathrm{C}$ shows the mean Fano factor across 
populations as a function of window size (10-200 ms), indicating that neural spiking in cortical area V4 was more variable across trials than in the thalamic populations independently of the size of the window in which spikes were counted. Moreover, trial-to-trial variability during initial fixation and the magnitude of subsequent variability quenching following stimulus onset was significantly correlated on a site-bysite basis in V4 (Pearsons correlation coefficient $r=-0.49, p=1.74 \mathrm{e}-7$ ), but not in the thalamic regions that did not show significant quenching (Pearsons correlation coefficients, vPul: $r=-0.14, p=0.11$; $d P u l$ : $r=-0.11, p=0.17 ;$ LGN: $r=-0.06, p=0.51$ ). These results suggest that even prior to the onset of a visual stimulus sizeable differences exist between cortical and thalamic spiking variability that may impact subsequent quenching behaviour.

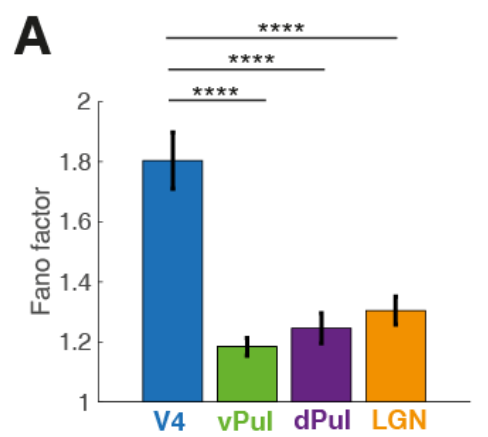

B

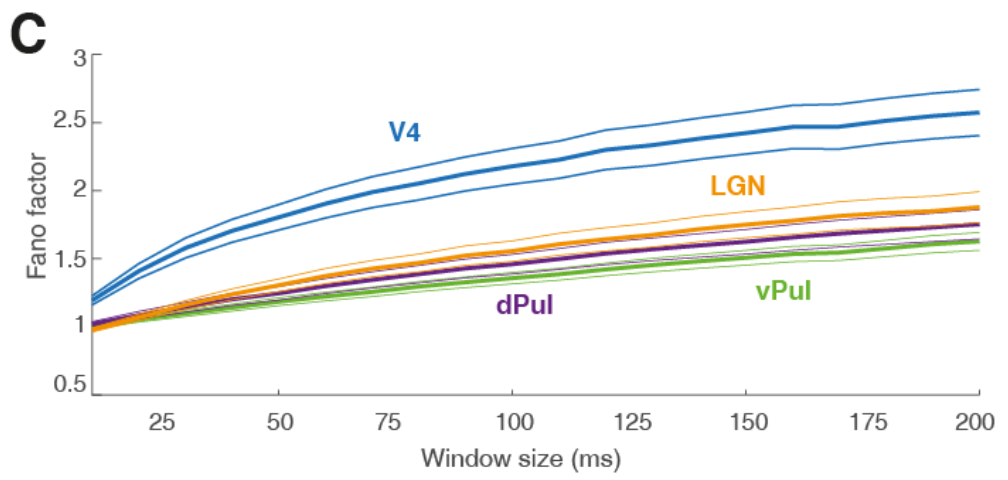

Figure 6. Trial-to-trial variability differences prior to stimulus onset. (A) Mean Fano factor calculated using non-overlapping $50 \mathrm{~ms}$ windows during the time interval of interest per region ( $\mathrm{V} 4 \mathrm{~N}=$ 100 , vPul $N=118$, dPul $N=136$, LGN N = 105). Error bars indicate +/- 1 SEM. Wilcoxon signed-rank tests, Bonferroni corrected, ${ }^{* * *} p<0.0001$. (B) Mean firing rates during the ongoing activity interval as a function of brain region (V4 N =100, vPul $N=118, d P u l ~ N=136, L G N ~ N=105)$. (C) Fano factor as a function of counting window size in all brain regions (V4 $N=100$, vPul $N=118, d P u l N=136, L G N ~ N$ = 105). Error bars indicate +/- 1 SEM. 


\begin{tabular}{|c|c|c|c|c|}
\hline & V4 & vPul & dPul & LGN \\
\hline $\mathrm{N}$ & 100 & 118 & 136 & 105 \\
\hline $\begin{array}{c}\text { mean Fano factor } \\
\text { +/- SD }\end{array}$ & $1.80+/-0.93$ & $1.17+/-0.29$ & $1.25+/-0.58$ & $1.30+/-0.48$ \\
\hline $\begin{array}{c}\text { mean firing rate } \\
\text { (spikes/s) +/- SD }\end{array}$ & $46+/-65$ & $38+/-62$ & $32+/-36$ & $46+/-61$ \\
\hline
\end{tabular}

Table 2. Mean Fano factors and firing rates $+/$ - standard deviation during ongoing activity $1000-500$ ms prior to target stimulus onset. $\mathrm{N}$ denotes the number of recording sites.

\subsection{Discussion}

As expected from previous studies (Mitchell et al., 2007, 2009; Cohen and Maunsell, 2009; Churchland et al., 2010) visual stimulation significantly reduced trial-to-trial variability in area V4. In the pulvinar and LGN, we found the variability quenching effect to be either absent or, in case of the dorsal pulvinar, marginal compared to the well-described variability decline in cortex, even when firing rate distributions were matched. In the ventral portion of the pulvinar, which exchanges recurrent connections with extrastriate cortex including area $\mathrm{V} 4$, the quenching effect was entirely absent despite it likely receiving a large portion of inputs from V4.

While we observed a slightly lower response variability in the pulvinar compared to visual cortex, spiking activity in LGN and pulvinar was also significantly less variable than in area V4 during steady fixation when visual input did not change. This comparatively reliable activity in the thalamus could in theory account for the lack of stabilization by stimulus onset as spiking variability is already low even when the cells are not driven by external input and may simply not be further reduced due to natural constraints underlying spike generation. One of the simplest models of neural spiking is a Poisson process, which is characterized by spikes occurring independently of each other, resulting in the spike count variance being equal to the mean (Fano factor $=1$ ). Although low or even sub-Poisson Fano factor values have been measured in some instances (Kara et al., 2000), cortical spiking is typically reported to show a considerable amount of additional variance, which can largely be explained by excitability fluctuations that are correlated over time and between neurons (Schölvinck et al., 2015; Malina et al., 2016). Assuming that spiking noise is approximately Poisson, there may be an irreducible lower bound to variability in neural systems that limits the impact of external input on neural populations that inherently show little excess variance (Goris et al., 2014). Since neural activity likely arises from an interaction 
between ongoing activity and stimulus-driven responses, it is possible that the pulvinar does not exhibit substantial quenching behaviour because variability is negligible even before stimulus onset. The fact that the V4 sites that were least variable prior to stimulus onset also showed the smallest magnitude of quenching supports this view.

Previous studies proposed that the stimulus-induced reduction of firing rate variability may constitute a general property of large recurrent networks (Churchland et al., 2010) and can naturally arise from a pattern of balanced inhibition and excitation in an attractor network (Deco and Hugues, 2012). While the network switches between multiple attractor states as long as it is not externally driven, excitatory input stabilizes one specific attractor and thus reduces spiking variability within the network. Given that both LGN and pulvinar do not show an equivalent decrease in variability, it is possible that differences in local network architecture between thalamus and cortex explain the stable activity within the thalamus. For example, while neocortical neurons are extensively interconnected in a highly specific manner (Harris and Shepherd, 2015), there is no evidence for direct connections between relay cells in the thalamus (Sherman, 2017).

We observed a small but significant quenching effect in the dorsal pulvinar in response to the target stimulus which was followed by a hand movement, but not to the RDM stimulus which did not prompt a behavioural response by the animal. Closer inspection revealed no significant difference in trial-to-trial variability before and after the movement. However, variability changes in premotor cortex have been shown to occur following relevant visual cues but prior to the movement(Churchland et al., 2006), suggesting that the quenching effect we observed in the dorsal pulvinar may have nonetheless been related to motor preparation as well.

Our results are consistent with an earlier study that found response variability in the pulvinar to be lower than in extrastriate visual cortex V4, although no comparisons of stimulus-induced variability changes were made(Bender and Youakim, 2001). Interestingly, the authors reported modulations of excitability associated with attentive fixation that occurred in both pulvinar and cortex and were of similar magnitude when variability differences between regions were taken into account. We did not manipulate attentional state in the current study, but it can be assumed that similar modulations of excitability occurred during the fixation period prior to the onset of the target stimulus that prompted a response by the animal. In addition to modulations of firing rate, attention has been associated with reduced trial-to-trial variability 
of V4 responses compared to unattended stimuli as well as during sustained attention (Cohen and Maunsell, 2009; Mitchell et al., 2009) and the magnitude of attentional modulation in the pulvinar has been found to be significantly smaller than in area V4 (Zhou et al., 2016) so it is likely that these differences in modulation strength and the differences in the degree of variability between cortex and pulvinar during fixation are closely linked. In addition, experimental evidence from rodents and primates suggests that the functional role of higher-order thalamic nuclei such as the pulvinar and the mediodorsal thalamus goes beyond that of a mere cortico-cortical relay and is critical for the flexible coordination of activity within and across cortical regions (Saalmann et al., 2012; Zhou et al., 2016; Halassa and Kastner, 2017; Schmitt et al., 2017; Fiebelkorn et al., 2019). In particular, the mediodorsal pulvinar has recently been shown to coordinate the fronto-parietal network directing spatial attention in macaques(Fiebelkorn et al., 2019). These findings are consistent with the view that the pulvinar and mediodorsal thalamus contain circuits that, rather than transmitting information from one cortical area to another, shift and sustain functional connectivity across cortex according to task demands (Halassa and Kastner, 2017).

Response variability in visual cortex has been shown to strongly depend on cortical state as excitability fluctuations that account for much of the variability in cortical responses as well as spontaneous activity are largest in synchronized states (Schölvinck et al., 2015). The significantly lower variability in the pulvinar compared to V4 we observed suggests that the impact of these fluctuations on spiking activity in the pulvinar are minimal despite its extensive reciprocal connections with the cerebral cortex.

\section{Data availability}

The datasets analyzed during the current study are available from the corresponding author on reasonable request.

\section{Acknowledgements}

This work was supported by the Hermann and Lilly Schilling Foundation, German Research Foundation (DO 1240/3-1), NIMH, NINDS and NEI Intramural Research Program. 


\subsection{References}

Acuna C, Cudeiro J, Gonzalez F, Alonso JM, Perez R (1990) Lateral-posterior and pulvinar reaching cells--comparison with parietal area 5a: a study in behaving Macaca nemestrina monkeys. Exp Brain Res 82:158-166.

Arieli A, Sterkin A, Grinvald A, Aertsen A (1996) Dynamics of ongoing activity: explanation of the large variability in evoked cortical responses. Science 273:1868-1871.

Bender DB, Youakim M (2001) Effect of attentive fixation in macaque thalamus and cortex. $J$ Neurophysiol 85:219-234.

Benevento LA, Port JD (1995) Single neurons with both form/color differential responses and saccade-related responses in the nonretinotopic pulvinar of the behaving macaque monkey. Vis Neurosci 12:523-544.

Berman RA, Wurtz RH (2008) Exploring the pulvinar path to visual cortex. Prog Brain Res 171:467473.

Bickford ME (2015) Thalamic Circuit Diversity: Modulation of the Driver/Modulator Framework. Front Neural Circuits 9:86.

Bridge H, Leopold DA, Bourne JA (2016) Adaptive pulvinar circuitry supports visual cognition. Trends in cognitive sciences 20:146.

Churchland MM et al. (2010) Stimulus onset quenches neural variability: a widespread cortical phenomenon. Nat Neurosci 13:369-378.

Churchland MM, Yu BM, Ryu SI, Santhanam G, Shenoy KV (2006) Neural Variability in Premotor Cortex Provides a Signature of Motor Preparation. J Neurosci 26:3697-3712.

Cohen MR, Kohn A (2011) Measuring and interpreting neuronal correlations. Nat Neurosci 14:811819.

Cohen MR, Maunsell JHR (2009) Attention improves performance primarily by reducing interneuronal correlations. Nature Neuroscience 12:1594-1600.

Deco G, Hugues E (2012) Neural Network Mechanisms Underlying Stimulus Driven Variability Reduction. PLOS Comput Biol 8:e1002395.

Denève S, Machens CK (2016) Efficient codes and balanced networks. Nat Neurosci 19:375-382.

Dinstein I, Heeger DJ, Behrmann M (2015) Neural variability: friend or foe? Trends in Cognitive Sciences 19:322-328.

Dominguez-Vargas A-U, Schneider L, Wilke M, Kagan I (2017) Electrical Microstimulation of the Pulvinar Biases Saccade Choices and Reaction Times in a Time-Dependent Manner. J Neurosci 37:2234-2257.

Fiebelkorn IC, Pinsk MA, Kastner S (2019) The mediodorsal pulvinar coordinates the macaque frontoparietal network during rhythmic spatial attention. Nature Communications 10:215.

Gattass R, Galkin TW, Desimone R, Ungerleider LG (2014) Subcortical connections of area V4 in the macaque. J Comp Neurol 522:1941-1965.

Goris RLT, Movshon JA, Simoncelli EP (2014) Partitioning neuronal variability. Nat Neurosci 17:858865.

Guillery RW, Sherman SM (2002) Thalamic relay functions and their role in corticocortical communication: generalizations from the visual system. Neuron 33:163-175. 
Gutierrez C, Cola MG, Seltzer B, Cusick C (2000a) Neurochemical and connectional organization of the dorsal pulvinar complex in monkeys. J Comp Neurol 419:61-86.

Gutierrez C, Cola MG, Seltzer B, Cusick C (2000b) Neurochemical and connectional organization of the dorsal pulvinar complex in monkeys. J Comp Neurol 419:61-86.

Halassa MM, Kastner S (2017) Thalamic functions in distributed cognitive control. Nat Neurosci 20:1669-1679.

Harris KD, Shepherd GMG (2015) The neocortical circuit: themes and variations. Nat Neurosci 18:170-181.

Jones EG (2012) The Thalamus. Springer Science \& Business Media.

Kaas JH, Lyon DC (2007) Pulvinar contributions to the dorsal and ventral streams of visual processing in primates. Brain Res Rev 55:285-296.

Kara P, Reinagel P, Reid RC (2000) Low Response Variability in Simultaneously Recorded Retinal, Thalamic, and Cortical Neurons. Neuron 27:635-646.

Komura Y, Nikkuni A, Hirashima N, Uetake T, Miyamoto A (2013) Responses of pulvinar neurons reflect a subject's confidence in visual categorization. Nature Neuroscience 16:749-755.

Malina KC-K, Mohar B, Rappaport AN, Lampl I (2016) Local and thalamic origins of correlated ongoing and sensory-evoked cortical activities. Nature Communications 7:ncomms12740.

Mitchell JF, Sundberg KA, Reynolds JH (2007) Differential attention-dependent response modulation across cell classes in macaque visual area V4. Neuron 55:131-141.

Mitchell JF, Sundberg KA, Reynolds JH (2009) Spatial Attention Decorrelates Intrinsic Activity Fluctuations in Macaque Area V4. Neuron 63:879-888.

Müller K-M, Wilke M, Leopold DA (2009) Visual adaptation to convexity in macaque area V4. Neuroscience 161:655-662.

Renart A, Machens CK (2014) Variability in neural activity and behavior. Current Opinion in Neurobiology 25:211-220.

Robinson DL, Petersen SE (1992) The pulvinar and visual salience. Trends Neurosci 15:127-132.

Robinson DL, Petersen SE, Keys W (1986) Saccade-related and visual activities in the pulvinar nuclei of the behaving rhesus monkey. Exp Brain Res 62:625-634.

Saalmann YB, Kastner S (2011) Cognitive and Perceptual Functions of the Visual Thalamus. Neuron 71:209-223.

Saalmann YB, Pinsk MA, Wang L, Li X, Kastner S (2012) The pulvinar regulates information transmission between cortical areas based on attention demands. Science 337:753-756.

Sadagopan S, Ferster D (2012) Feedforward Origins of Response Variability Underlying Contrast Invariant Orientation Tuning in Cat Visual Cortex. Neuron 74:911-923.

Schmitt LI, Wimmer RD, Nakajima M, Happ M, Mofakham S, Halassa MM (2017) Thalamic amplification of cortical connectivity sustains attentional control. Nature 545:219-223.

Schölvinck ML, Saleem AB, Benucci A, Harris KD, Carandini M (2015) Cortical State Determines Global Variability and Correlations in Visual Cortex. J Neurosci 35:170-178.

Shadlen MN, Britten KH, Newsome WT, Movshon JA (1996) A computational analysis of the relationship between neuronal and behavioral responses to visual motion. J Neurosci 16:1486-1510.

Shadlen MN, Newsome WT (1998) The Variable Discharge of Cortical Neurons: Implications for Connectivity, Computation, and Information Coding. J Neurosci 18:3870-3896. 
Sherman SM (2017) Functioning of Circuits Connecting Thalamus and Cortex. Compr Physiol 7:713739.

Shipp S (2003) The functional logic of cortico-pulvinar connections. Philos Trans R Soc Lond B Biol Sci 358:1605-1624.

Softky WR, Koch C (1993) The highly irregular firing of cortical cells is inconsistent with temporal integration of random EPSPs. J Neurosci 13:334-350.

Stepniewska I (2004) The Pulvinar Complex. In: The Primate Visual System (Kaas JH\& C CE, ed), pp 53-80. London: CRC Press.

Wilke M, Mueller K-M, Leopold DA (2009) Neural activity in the visual thalamus reflects perceptual suppression. PNAS 106:9465-9470.

Wilke M, Turchi J, Smith K, Mishkin M, Leopold DA (2010) Pulvinar inactivation disrupts selection of movement plans. J Neurosci 30:8650-8659.

Yu C, Sellers KK, Radtke-Schuller S, Lu J, Xing L, Ghukasyan V, Li Y, Shih Y-YI, Murrow R, Frohlich F (2016) Structural and Functional Connectivity between the Lateral Posterior-Pulvinar Complex and Primary Visual Cortex in the Ferret. The European journal of neuroscience 43:230.

Zhou H, Schafer RJ, Desimone R (2016) Pulvinar-Cortex Interactions in Vision and Attention. Neuron 89:209-220.

Zohary E, Shadlen MN, Newsome WT (1994) Correlated neuronal discharge rate and its implications for psychophysical performance. Nature 370:140-143. 


\subsection{Supplementary}
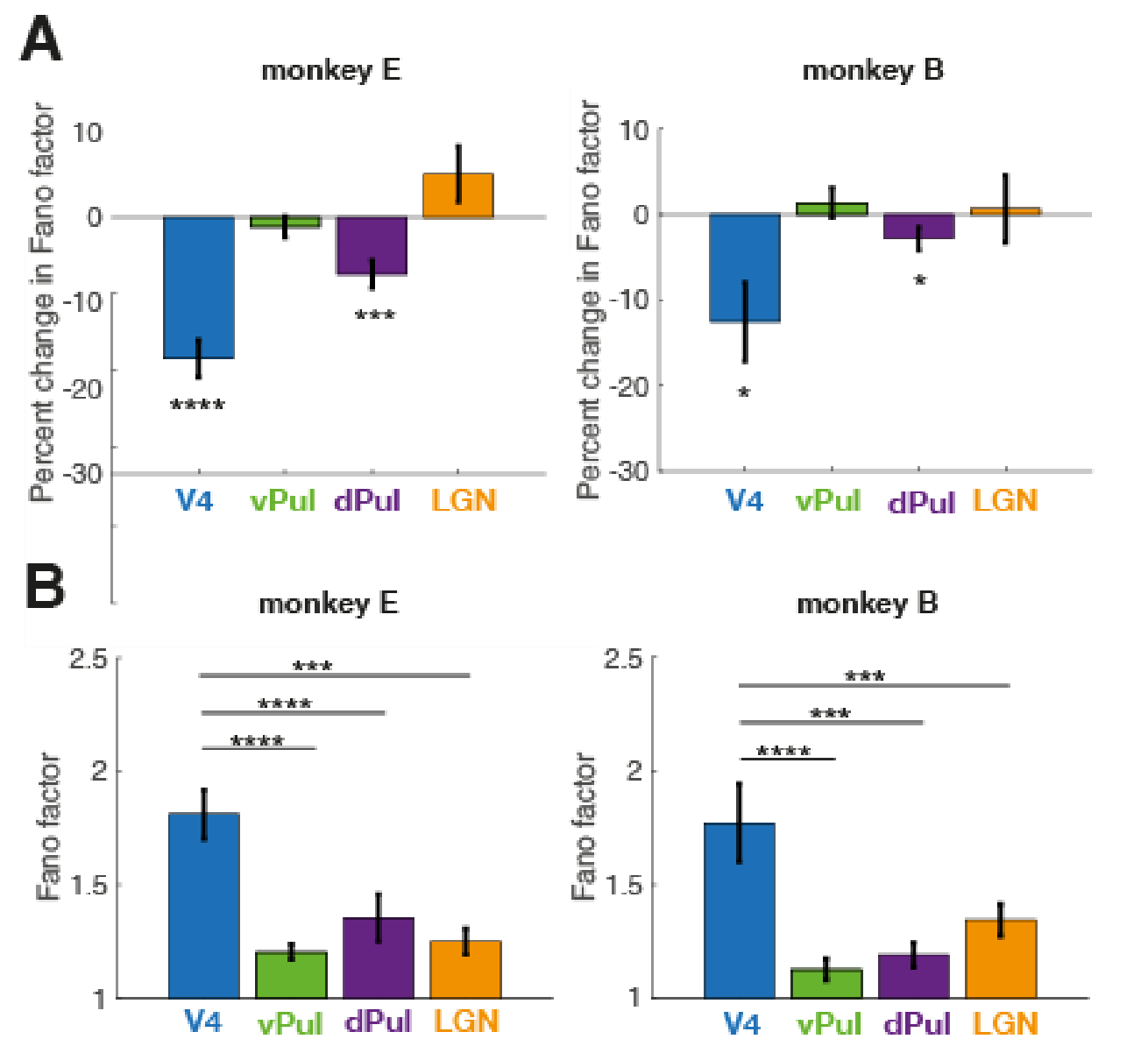

\section{Supplementary Information S1: Separate results of the two animals}

The main results of the current study, namely (1) the lack of quenching in the thalamic regions compared to cortical area V4, as well as (2) the significantly lower levels of trial-to-trial variability in the thalamic regions absence of changes in stimulus input, were remarkably similar between the two animals. The mean percent change in Fano factor with the onset of the target stimulus for the two monkeys is illustrated in Figure S1A. Both animals showed an individually significant quenching effect in area V4 (One-sample t-tests on the percent change in Fano factor with stimulus onset, monkey $E: p=2.23 e$ $11, N=85$, monkey $B: p=0.01, N=15)$. A significant decline in Fano factor in the dorsal pulvinar was also observed in both cases (One-sample t-tests, monkey $E: p=1.70 e-04$, monkey $B: p=0.03$ ), while the decreases were significantly smaller than those in V4 (Two-sample t-tests, monkey $E: p=2.42 e-3$, monkey $B: p=0.01$ ). A quenching effect was consistently absent in the ventral pulvinar portion (Onesample t-tests, monkey $E: p=2.23 e-11, N=72$, monkey $B: p=0.01, N=48$ ) as well as in the LGN (One-sample t-tests, monkey $E: p=2.23 e-11, N=45$, monkey $B: p=0.01, N=60$ ). Figure S1B shows the average Fano factors during the fixation period $1000-500 \mathrm{~ms}$ prior to target onset for both animals separately. The statistical comparisons between area V4 and all thalamic nuclei (vPul, dPul, LGN) were consistent between and individually significant in both monkeys (Wilcoxon rank-sum tests, V4-vPul: monkey $E \mathrm{p}=2.13 \mathrm{e}-06$, monkey $\mathrm{B} \mathrm{p}=2.07 \mathrm{e}-05$; V4-dPul: monkey $\mathrm{E} \mathrm{p}=4.18 \mathrm{e}-04$, monkey $\mathrm{B} p=1.81 \mathrm{e}$ 05; V4-LGN: monkey $E \mathrm{p}=1.40 \mathrm{e}-05$, monkey $\mathrm{B} p=0$. 35e-2), confirming the significantly lower variability in the thalamic regions compared to cortical area V4 prior to stimulus onset. 
A

single-unit activity (SUA)
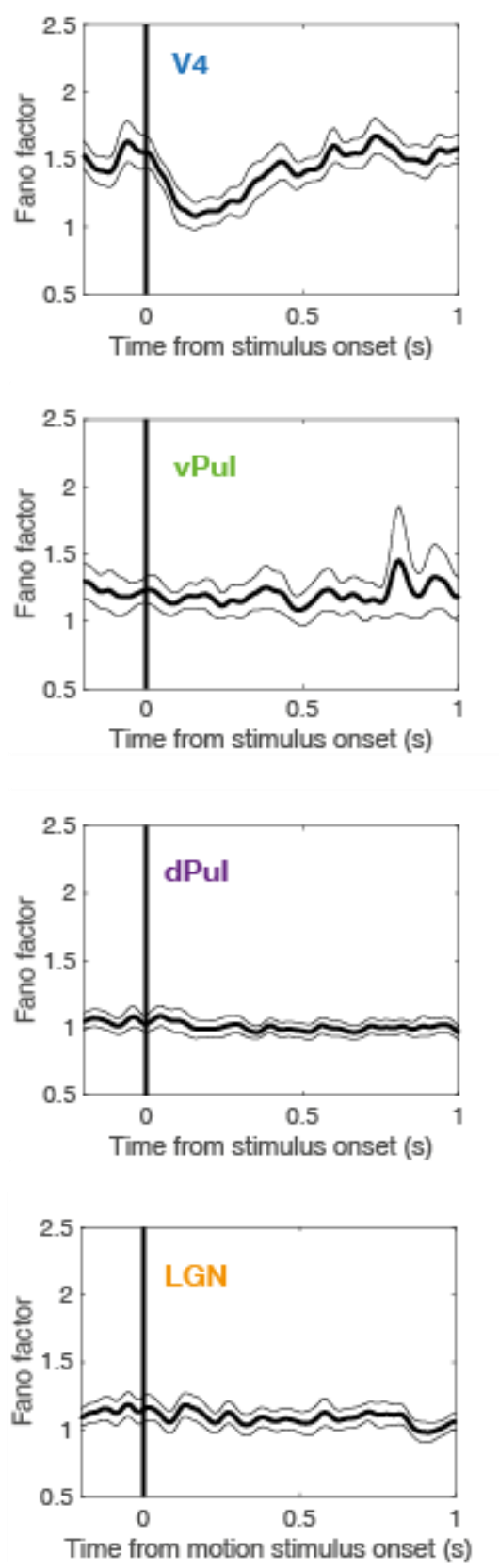

\section{B multi-unit activity (MUA)}
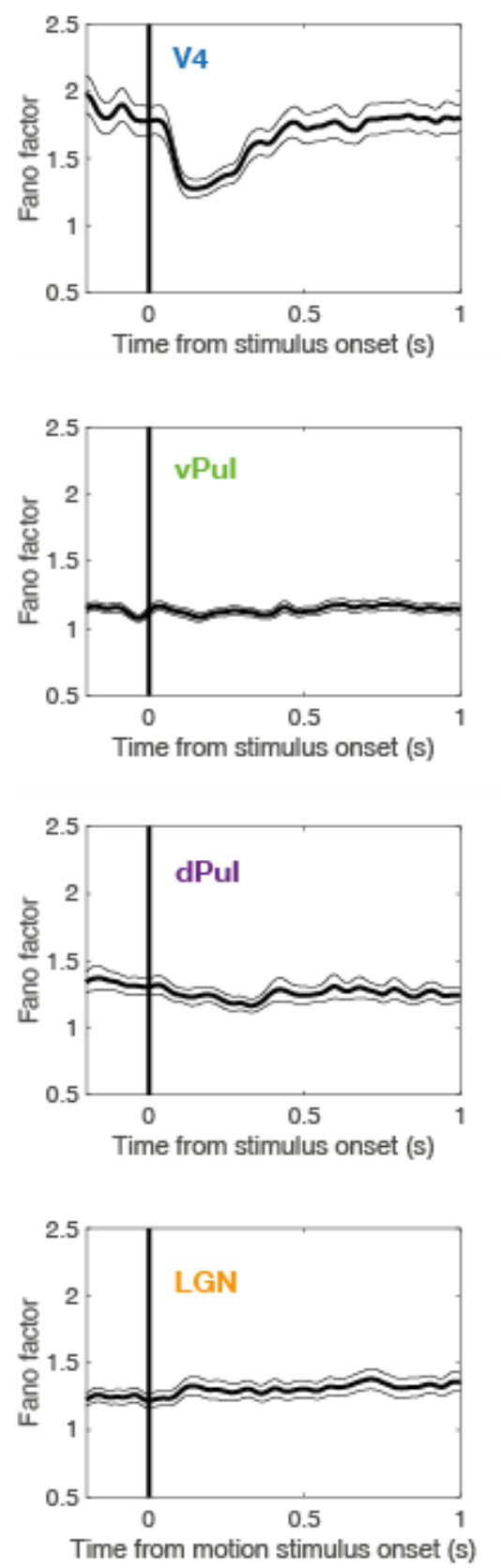

\section{Supplementary Information S2: Comparison of single- and multi-unit data}

Single- and multi-unit activity were pooled for the current study as they were generally very similar (Figure S2A-B). Restricting the analysis of variability changes with target onset to the few available single units confirmed the significant decrease in area V4, while it was absent in dorsal and ventral pulvinar as well as LGN cells (One-sample t-tests on the percent change in Fano factor with stimulus onset, V4 $\mathrm{N}=19, \mathrm{p}=4.00 \mathrm{e}-3$, vPul $\mathrm{N}=12, \mathrm{p}=0.33$, dPul $\mathrm{N}=34, \mathrm{p}=0.06$, LGN N = 16, $\mathrm{p}=0.39$ ). Similar results were obtained for the multi-unit data (One-sample t-tests, V4 $N=81, p=8.64 \mathrm{e}-11$, vPul $\mathrm{N}=108, \mathrm{p}=0.90$, dPul $\mathrm{N}=102, \mathrm{p}=8.69 \mathrm{e}-04, \mathrm{LGN} \mathrm{N}=89, \mathrm{p}=0.12$ ). Trial-to-trial variability during stable fixation was slightly lower in the single-unit data of V4 and the dorsal pulvinar than in the respective multi-unit activity, but there was no significant difference (Wilcoxon ranked-sum tests, V4: $p$ $=0.41, \mathrm{dPul}: \mathrm{p}=0.69$ ). 

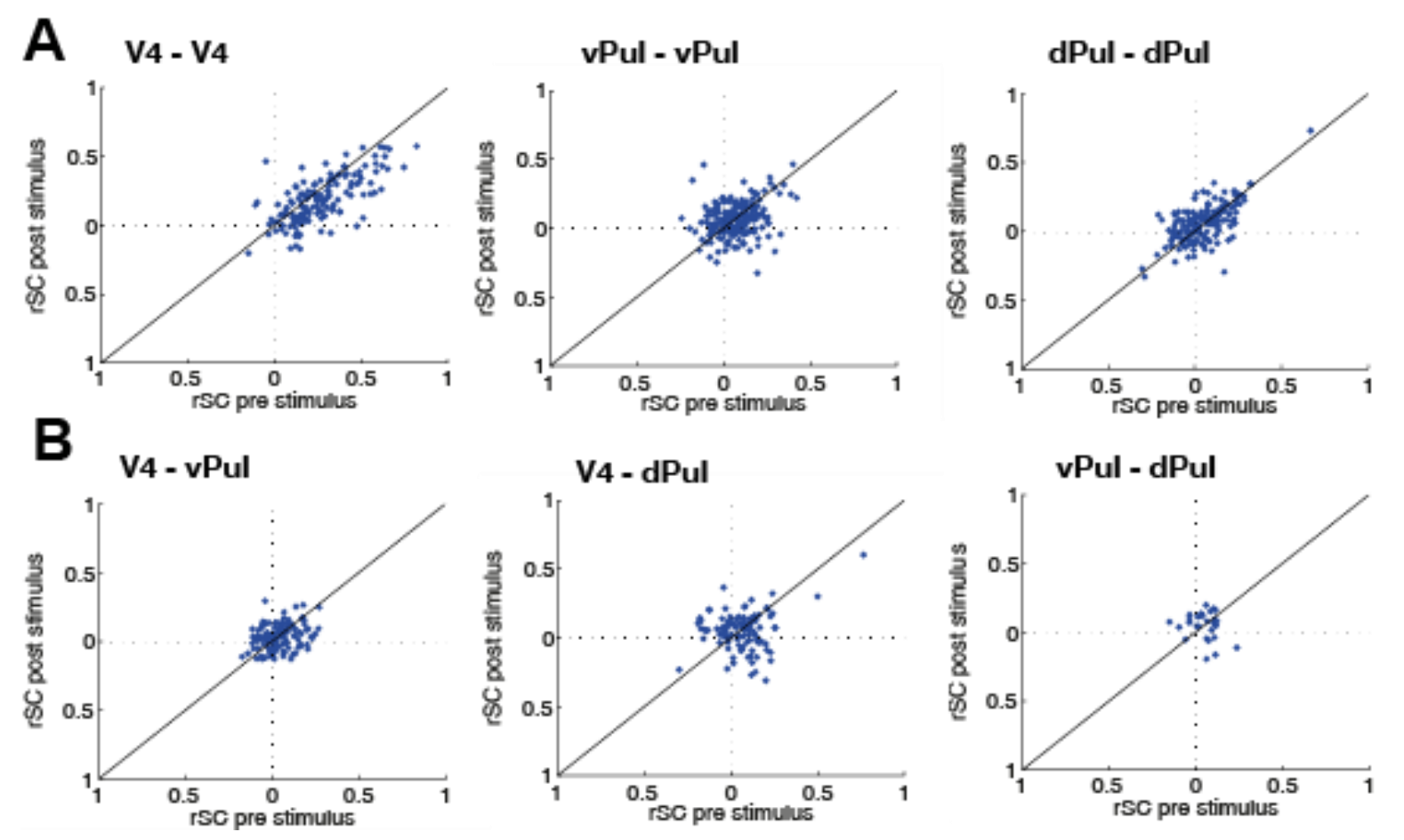

\section{Supplementary Information S3: Shared variability analysis}

We wondered to which degree the trial-to-trial variability we observed was shared between neurons and examined simultaneously recorded unit pairs within area V4 and the pulvinar (V4-V4 N = 141,vPulvPul $\mathrm{N}=201$, dPul-dPul $\mathrm{N}=174$ ) as well as between V4 and the pulvinar (V4-vPul $\mathrm{N}=117$, V4-dPul $\mathrm{N}$ $=109$, vPul-dPul $N=28$ ). For these pairs, we calculated the spike count correlations $\mathrm{rSC}$ (sometimes also referred to as noise correlations)as the Pearson's correlation coefficient of spike counts for the 300 $\mathrm{ms}$ pre and the $300 \mathrm{~ms}$ post target stimulus intervals. Within area V4, shared variability decreased significantly from pre to post stimulus interval (Wilcoxon signed-rank test, $p=5.29 \mathrm{e}-10$, Figure S3A). Within the dorsal and ventral pulvinar we did not observe any significant changes in spike count correlations with stimulus onset (Wilcoxon signed-rank tests, vPul-vPul $p=0.73$; dPul-dPul $p=0.91$; Figure S3A). There was no significant decrease in shared variability between regions (Wilcoxon signedrank tests, V4-vPul $p=0.52$; V4-dPul $p=0.94$; vPul-dPul $p=0.62$; Figure S3B). 
A

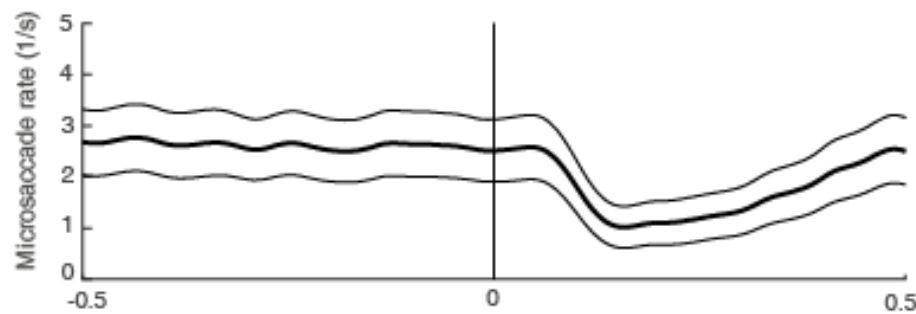

B

Time from stimulus onset (s)
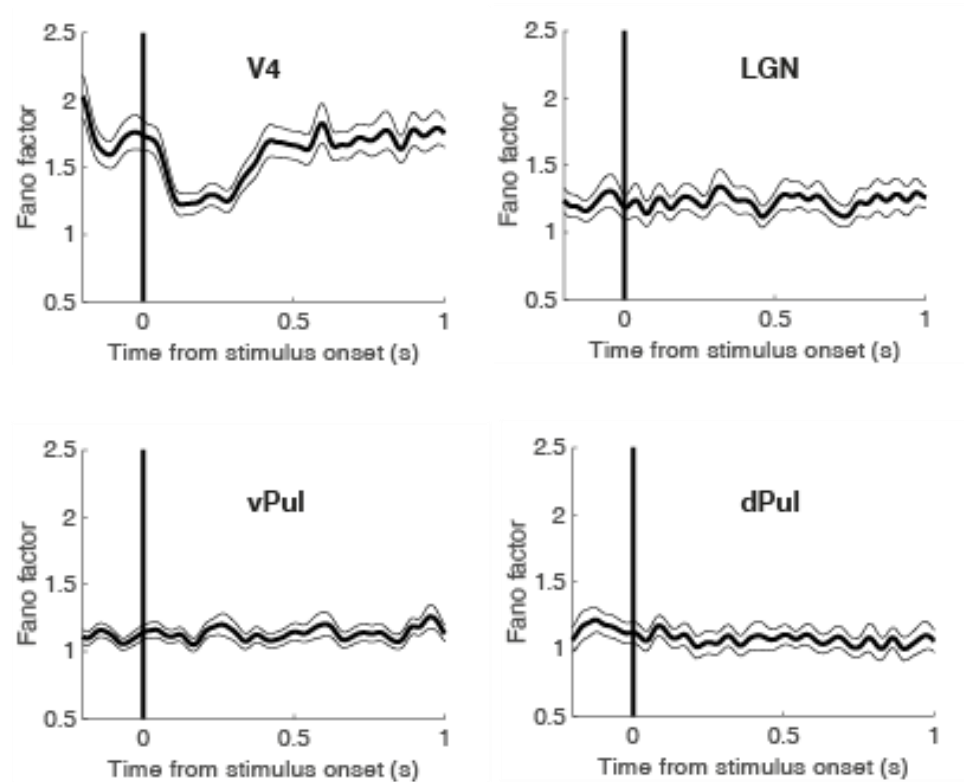

\section{Supplementary Information S4: Changes in microsaccade rate with stimulus onset}

Changes in neural trial-to-trial variability have previously been associated with small eye movements (microsaccades) that occur during fixation1. We thus aimed to assess the influence of microsaccades on the quenching effect. Consistent with a previous study (2), we found the microsaccade rate to decrease following the onset of the target stimulus (Figure S4A, $300 \mathrm{~ms}$ pre to $300 \mathrm{~ms}$ post target onset, Wilcoxon signed-rank test, $p=2.96 \mathrm{e}-10$ ). To investigate whether the stimulus-induced variability reduction in V4 and dorsal pulvinar could be attributed to changes in microsaccade rate we calculated the Fano factor for trials that did not contain microsaccades in the relevant trial period $-300 \mathrm{~ms}$ to $300 \mathrm{~ms}$ relative to target stimulus onset. In those microsaccade-removed trials a significant decrease of variability following target onset was still obtained in area V4 but not in either pulvinar subnucleus nor in the LGN (Figure S4B, Wilcoxon signed-rank tests, V4 $p=6.05 \mathrm{e}-09$, dPul $p=0.39$; vPul $p=0.58$; LGN $p=0.30$ ).

\section{References}

1. Gur, M., Beylin, A. \& Snodderly, D. M. Response variability of neurons in primary visual cortex (V1) of alert monkeys. J. Neurosci. Off. J. Soc. Neurosci.17,2914-2920 (1997).

2. Cui, J., Wilke, M., Logothetis, N. K., Leopold, D. A. \& Liang, H. Visibility states modulate microsaccade rate and direction. Vision Res.49,228-236(2009). 


\title{
Chapter III
}

\section{Trial-to-trial variability as a signature of conscious perception}

\author{
Eva Poland ${ }^{1}$, Tobias H. Donner², Kai-Markus Müller ${ }^{3}$, David A. Leopold ${ }^{4}$, Melanie Wilke1,5,6
}

\begin{abstract}
${ }^{1}$ Department of Cognitive Neurology, UMG, University Medicine Goettingen, Robert-Koch-Str. 40, Goettingen, 37075, Germany ${ }^{2}$ University Medical Center Hamburg-Eppendorf, UKE, Department of Neurophysiology and Pathophysiology, Building N43, Martinistr. 52, 20246 Hamburg, Germany

${ }^{3}$ Consumer Behavior, HFU Business School, Jakob-Kienzle-Str. 17, 78054 Villingen-Schwenningen, Germany

${ }^{4}$ Section on Cognitive Neurophysiology and Imaging, Laboratory of Neuropsychology, National Institute of Mental Health, National Institutes of Health, Building 49, Room B2J-45, MSC-4400 49 Convent Dr., Bethesda, MD 20892, USA

${ }^{5}$ DFG Center for Nanoscale Microscopy \& Molecular Physiology of the Brain (CNMPB), Robert-Koch-Str. 40, Göttingen, 37075, Germany

${ }^{6}$ German Primate Center, DPZ, Leibniz Institute for Primate Research, Kellnerweg 4, Goettingen, 37077, Germany
\end{abstract}

\section{Author contributions}

M. Wilke, T.H. Donner, D. A. Leopold and E. Poland developed the study concept, M. Wilke and K-M. Müller developed the task and collected the data. E. Poland analyzed the data. D. A. Leopold, T. H. Donner, E. Poland and M. Wilke interpreted the data and contributed to the analysis process. E. Poland wrote the manuscript. 


\subsection{Abstract}

Neural responses to repeated presentations of identical stimuli are highly variable and it has been debated whether spiking variability should be considered mere noise posing a limitation to sensory processing or whether it may contain information beyond the typically examined average firing rates. In favor of the latter hypothesis, previous research in humans has associated reduced trial-to-trial variability in EEG and fMRI BOLD signals with the conscious perception of visual stimuli. Here, we investigate whether trial-to-trial spiking variability reflects visual awareness in macaque visual cortex area V4 using a generalized flash suppression (GFS) paradigm. Contrasting physically identical trials on which a salient visual target stimulus is either consciously perceived or rendered subjectively invisible, we find trial-to-trial variability in area $\mathrm{V} 4$ to be significantly reduced during subjective target disappearances. Moreover, we replicate previous findings reporting perceptual modulations of firing rate and low frequency local field potential by GFS and compare them to the observed variability effects. Our results suggest that neural variability across trials indicates stimulus visibility, but may be a reflection of low frequency amplitude, particularly in the alpha $(8-12 \mathrm{~Hz})$ band, which is similarly reduced during perceptual suppression and correlates with spiking variability on a site-to-site basis.

\subsection{Introduction}

Spiking responses to the same physical stimuli as well as ongoing neural activity have been shown to vary to a considerable extent even under highly controlled experimental conditions (Softky and Koch, 1993; Shadlen and Newsome, 1998). Excessive neural variability is typically considered to constrain the reliability by which sensory information can be encoded (Britten et al., 1992; Zohary et al., 1994; Shadlen et al., 1996; Parker and Newsome, 1998), although the question whether the precise timing of individual spikes and spiking variability across trials itself may contain additional information besides the average number of spikes in a given time interval has been the subject of a long-standing debate (Shadlen and Newsome, 1998; Stein et al., 2005; Scaglione et al., 2011).

Trial-to-trial spiking variability, typically measured as the ratio between the variance and the mean of spike counts, has been shown to be reduced for attended stimuli compared to stimuli that were 
unattended (Mitchell et al., 2007, 2009; Cohen and Maunsell, 2009) and to strongly decrease upon stimulus onset, an effect commonly termed variability quenching that is observed throughout a wide range of cortical areas (Churchland et al., 2010). Moreover, the variability of various neural signals across trials has been related to perceptual performance (Schurger et al., 2010a, 2015a; Scaglione et al., 2011; Ledberg et al., 2012; Arazi et al., 2017; Ni et al., 2018) and a particularly compelling case has been made for a potential role of neural trial-to-trial variability in conscious perception: Visual stimuli that were presented at the perceptual threshold were associated with stronger variability quenching in the human EEG when they were consciously perceived compared to stimuli the subjects failed to detect (Schurger et al., 2015b), and examining trial-to-trial variability of human fMRI BOLD responses in a dichoptic color masking paradigm, activation patterns across repeated stimulus presentations were found to be less variable when stimuli were subjectively visible (Schurger et al., 2010b). Does the trialto-trial variability of neural signals reliably indicate visual awareness as such or can the observed differences be explained as a byproduct of changes in mean activity?

In the current study, we examine whether trial-to-trial variability of spiking responses recorded from macaque visual area V4 reflects target visibility during generalized flash suppression (GFS), a visual paradigm during which salient visual stimuli are suppressed from awareness on a subset of trials by the onset of a surrounding motion stimulus. We further compare perceptual suppression effects on spiking variability to the established perceptual modulations of mean firing rate and low frequency LFP amplitude. Previous studies contrasting neural activity corresponding to different states of awareness during GFS observed perceptual modulations of firing rate as well as local field potential (LFP) power in macaque visual cortex and higher-order visual thalamus (Wilke et al., 2006; Maier et al., 2008; Wilke et al., 2009a). The effect of subjective target invisibility on firing rates in area V4 was of the same order as the response to physical removal of the target stimulus, and the effect directionality was dependent on the directionality of the physical removal response, either exhibiting rate increases or rate decreases consistent with the responsiveness of individual sites (Wilke et al., 2006). Interestingly, firing rates did not reliably differentiate between perceptual outcomes in early visual cortex areas V1 and V2, while modulations of low frequency LFP, consistently showing reduced power in the alpha and beta range during perceptual suppression, were observed throughout striate as well as extrastriate visual cortex and the pulvinar (Wilke et al., 2006, 2009b; Maier et al., 2008). 


\subsection{Methods}

\section{Electrophysiological recordings}

Electrophysiological data were recorded from area V4 in two rhesus macaques (Macaca mulatta, monkey E and B). Spiking activity and local field potential were collected simultaneously with the MAP recording system (Plexon Inc., Dallas TX, USA) during a total of 113 recording sessions (62 monkey E, 51 monkey B) with 4 to 8 microelectrodes simultaneously (Thomas Recording GmbH, Giessen, Germany). Local field potential data were band-pass filtered between $1-100 \mathrm{~Hz}$ and digitized at $1 \mathrm{kHz}$. We only considered uninterrupted recordings with a minimum firing rate of 5 spikes/s and a minimum number of 10 trials in each perceptual condition, resulting in a data set of $93 \mathrm{~V} 4$ sites. All experiments were conducted following the guidelines of the National Institutes of Health (Bethesda MD, USA).

\section{Stimuli and task}

Electrophysiological data were collected in the context of a generalized flash Suppression (GFS) paradigm (Wilke et al., 2009). Stimuli were displayed on $38 \times 65 \mathrm{~cm}$ monitors using a mirror stereoscope. The screen to eye distance was $88 \mathrm{~cm}$. A small fixation spot $\left(0.15^{\circ}\right)$ was always presented in the middle of the screen and monkeys were required to maintain fixation within a radius of $0.7^{\circ}$ visual angle before the target stimulus appeared (Figure 1B). Target stimuli consisted either of a red disk or grating of $0.3^{\circ}$ to $6^{\circ}$ visual angle and the eccentricity of the targets $\left(0.3^{\circ}-7.7^{\circ}\right)$ was varied on a session basis depending on the position of the receptive fields (RF) of the best isolated recorded neurons. Following target onset, monkeys had to pull a lever. After the target stimulus was shown for $2 \mathrm{~s}$, a fullfield random dot motion (RDM) surround stimulus ( 70 to 240 white dots on a black background moving at a speed of $10.8 \% \mathrm{~s}$ ) was presented. The data set consisted of test ('ambiguous') trials as well as catch ('unambiguous') trials. In test trials the target was presented monocularly and, depending on the session, the surround pattern was presented either binocularly or to the opposite eye, thus inducing perceptual suppression of the target stimulus in a subset of trials. During test trials, monkeys were permitted to release the lever at any time after the onset of the surround or to hold the level upon end of the trial to indicate target visibility. In all test cases, stimuli were physically identical and were sorted offline based on the reported target visibility. Visible denotes the trials were monkeys kept holding the level at least up to 2000 ms following surround onset and invisible when the lever was released within 
$800 \mathrm{~ms}$ following surround onset. In order to ensure that the monkeys performed the task correctly, test trials were interleaved with a higher number of catch trials (3:1 to $6: 1$ ratio), where ocular configurations were either adjusted to minimize perceptual suppression by showing the target to the same eye as the surround ('visible control') or the target was physically removed at a variable time following surround onset ('physical removal').

\section{Spiking data analysis}

Neurophysiological data were processed and analysed offline using custom-written software in MATLAB 2015b (The MathWorks Inc., Natick MA, USA). For each recording site, we determined responsiveness to the target stimulus, the RDM stimulus as well as the physical removal of the target stimulus with Wilcoxon rank-sum tests $(p<0.05)$ between a $100 \mathrm{~ms}$ window immediately preceding stimulus onset and a $100 \mathrm{~ms}$ window at a latency between 50 and $150 \mathrm{~ms}$ after stimulus onset that yielded the maximal change. The Fano factor was calculated as the variance divided by the mean of spike counts for each recording site and interval of interest. For graphical representation of trial-to-trial variability over time the Fano factor was computed using a 50 ms sliding window moving in $10 \mathrm{~ms}$ steps and then smoothed using a moving average. For statistical analysis, the Fano factor was calculated in non-overlapping $50 \mathrm{~ms}$ windows covering the respective trial interval and averaged to obtain a single value per interval. For the analysis of the effects of perceptual suppression, we compared visible and invisible ambiguous test trials. For each recording, we determined the greatest common number of trials in each perceptual condition and averaged results over 100 repetitions of randomly sampled trials from perceptual conditions that exceeded the common number of trials. Mean trial numbers per perceptual condition were 26 . For statistical analysis, we initially focused on a trial interval ranging from $300-800$ ms after surround onset, which was also used for analysis of perceptual suppression effects on mean firing rates in previous work (Wilke et al., 2009b). We calculated average firing rates and firing rates in percent change from baseline (300 - 0 ms prior to surround onset) as well as average Fano factors for visible and invisible test trials. We adapted the distribution-matching procedure developed in a previous study (Churchland et al., 2010) to equate firing rate distributions between visible and invisible trials at a resolution of 10 spikes/s ( $N=43$ data points). To simulate equal mean firing rates between visible and invisible trials, we calculated mean spike counts of non-overlapping $50 \mathrm{~ms}$ windows spanning the 300 - 800 ms post stimulus interval for each recording site. We then determined the greatest firing rate 
distribution common to both perceptual conditions for each window and calculated the Fano factor based on randomly selected subpopulations of sites yielding the determined greatest common firing rate distribution for each time window. We then averaged the results across 1000 repetitions of random selection of suitable data points. Statistical significance of the difference between perceptual outcomes was assessed based on the $300-800$ ms post surround interval using Wilcoxon signed-rank tests. We estimated the onset of the perceptual modulations by performing Wilcoxon signed-rank tests between visible and invisible conditions in a $50 \mathrm{~ms}$ sliding window moving in $10 \mathrm{~ms}$ steps that covered the second following RDM stimulus onset and correcting for multiple comparisons (Bonferroni correction, $\mathrm{p}<$ 0.0005). For comparison to local field potential (LFP) amplitude, we calculated the average Fano factor across ambiguous test trials in a $500-1000$ ms post RDM stimulus interval that best captured both the modulation of trial-to-trial variability and low frequency amplitudes.

\section{Local field potential (LFP) data analysis}

The simultaneously recorded local field potential data was analysed using custom-written software in MATLAB 2015b (The MathWorks Inc., Natick MA, USA) as well as the Fieldtrip toolbox (Oostenveld et al., 2011). We first calculated the Fast Fourier Transform (FFT) over the $500-1000$ ms post RDM stimulus interval over a frequency range of $3-30 \mathrm{~Hz}$ for visible and invisible trials separately using a Hanning window. Due to the 500 ms window constraining the number of cycles on which the power of very low frequency oscillations could be estimated, we did not investigate frequencies $<3 \mathrm{~Hz}$. Next we grouped the low frequency range into the theta $(4-7 \mathrm{~Hz})$, alpha $(8-12 \mathrm{~Hz})$ and beta $(13-20 \mathrm{~Hz})$ frequency bands by band-pass filtering the data using a $4^{\text {th }}$ order Butterworth filter and subsequently calculated the amplitude of the respective band, i.e. the envelope of the filtered signal, using an absolute Hilbert transform for visible and invisible trials. We employed the same sliding window approach to determine the respective onsets of perceptual modulation of low frequency amplitude in the different frequency bands. Significant differences between perceptual conditions were assessed based on the average amplitude in the $500-100 \mathrm{~ms}$ post RDM interval. Finally, we calculated average theta, alpha and beta amplitudes during the $500-100$ ms post RDM interval across visible as well as invisible ambiguous test trials and calculated the Pearson's correlation coefficient to test for a significant correlation between average Fano factors and low frequency amplitudes. We employed a Bonferroni correction of $p<0.0167$ for tests performed on the three frequency bands. 


\subsection{Results}

In the current study, we asked whether trial-to-trial variability reflects visual awareness during perceptual suppression. To this end we employed a generalized flash suppression (GFS) paradigm allowing us to compare trials with the same physical stimuli that resulted in two different perceptual outcomes. Each trial began with a central fixation cross, followed by the onset of a salient target stimulus consisting of a red disk or grating that was shown to only one eye using a mirror stereoscope (Figure 1). After 2 seconds of target presentation, a surrounding stimulus consisting of random dot motion (RDM) was shown to the other eye, inducing the subjective disappearance of the target on a subset of trials.

We first evaluated whether V4 firing rates themselves reflected whether the visual target stimulus was suppressed from conscious perception by comparing average rates between visible and invisible trials. The majority of $\mathrm{V} 4$ sites in the examined population showed significant responses to both the target and the RDM surround stimulus onset (Wilcoxon ranked sum tests, $p<0.05$ ), with 63 out of the 93 sites being target-responsive and 71 out of 93 sites showing significant responses to RDM stimulus onset. Based on the reaction times obtained from the control trials in which the target was physically removed (mean RT across sessions $325 \mathrm{~ms}$ +/- SD $72 \mathrm{~ms}$ ) we estimated the mean times of subjective target disappearance by subtracting the mean RT of each session from the report times in the invisible test trials. The mean estimated onset of perceptual suppression across sessions was $218 \mathrm{~ms}$ +/- SD $56 \mathrm{~ms}$.

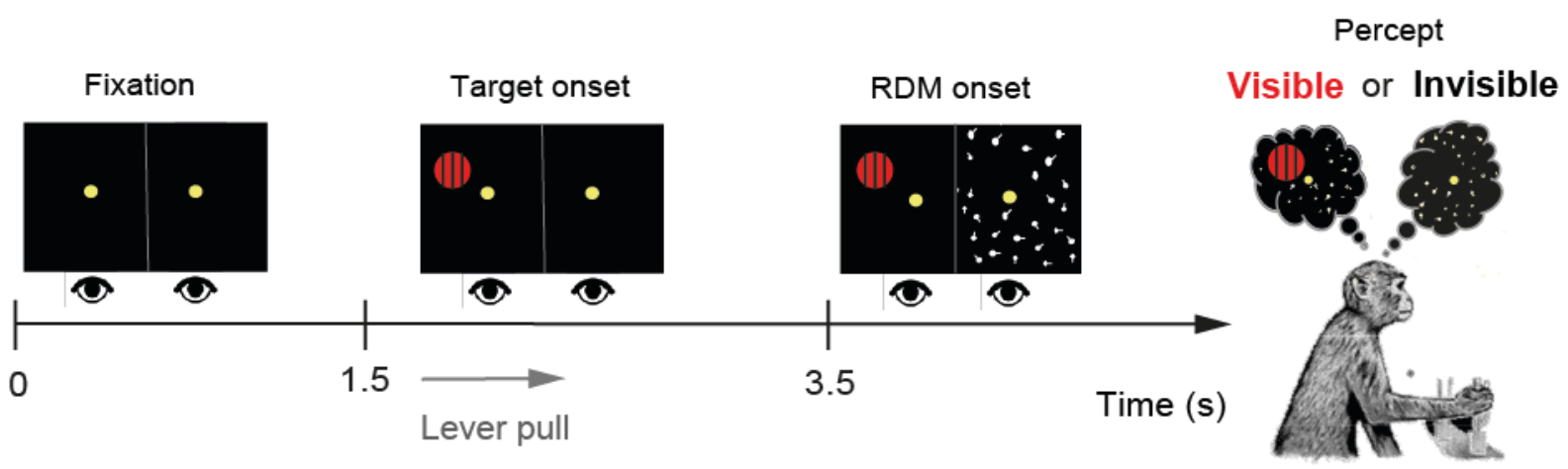

Figure 1. Generalized Flash Suppression (GFS) paradigm. Visual stimuli were presented through a mirror stereoscope in order to separate input to the left and right eyes. Each trial began with acquisition of the central fixation spot. $1.5 \mathrm{~s}$ later the target stimulus (red disk or grating) was shown. Target stimuli were either shown to the left or to the right eye. Monkeys were required to pull the lever upon target presentation and to continue holding it as long as the target was visible. $2 \mathrm{~s}$ after target onset, a surround stimulus consisting of randomly moving dots was added to the respective opposite eye. The onset of the random dot motion (RDM) stimulus caused the target to either subjectively disappear or to remain visible, which was reported by the monkey by releasing or continuing to hold the lever, respectively. 
For statistical analysis, we considered a post RDM stimulus interval ranging from 300 to $800 \mathrm{~ms}$ post RDM onset as used in previous work (Wilke, 2009), that likely captured an interval of sustained subjective suppression of the target on invisible trials. As expected from previous studies (Wilke et al., 2009), we found firing rates to be significantly modulated in the full V4 population (rate visible 2.65 spikes/s +/- SD 3.50, rate invisible 2.96 spikes/s +/- SD 3.72, Wilcoxon signed-rank test, $p=1.99 \mathrm{e}-10$, $\mathrm{N}=93$ ), showing greater activity during the sustained response period on trials on which the target had been suppressed (Figure 2A). We then evaluated whether V4 trial-to-trial spiking variability similarly differentiated between perceptual outcomes. To this end we calculated the Fano factor, a measure of variability across trials expressing the variance to mean ratio of spike counts, for visible and invisible test trials separately (Figure 2B). Perceptual suppression of the target coincided with a lower trial-totrial variability in area V4 during the $300-800$ ms post RDM window (Fano factor visible 1.38 +/- SD 0.59, Fano factor invisible $1.27+/-$ SD 0.52 , Wilcoxon signed-rank test, $p=1.82 \mathrm{e}-05, \mathrm{~N}=93$ ). In order to ensure that the modulation of spiking variability did not simply arise from firing rate differences between perceptual outcomes, we adapted the mean-matching procedure previously developed by Churchland and colleagues (Churchland et al., 2010) in order to equate firing rate distributions between visible and invisible trials during the post surround interval. We then based our statistical analysis on the matched data. The mean Fano factor for matched firing rates is shown in Figure 2C. The modulation of spiking variability was significant in rate-matched V4 populations (Wilcoxon signed-rank tests, $p=$ $1.65 \mathrm{e}-04, \mathrm{~N}=43$ data points).

Previous studies investigating modulations of spiking activity related to perceptual suppression in striate and extrastriate visual cortex as well as in the visual thalamus showed that the directionality of the effect was dependent on the response to the physical removal of the target: Sites which decreased their firing rates when the target was removed from screen also showed reduced activity during perceptual suppression, whereas sites that responded with rate increases showed higher rates on trials in which the target was suppressed from awareness (Wilke et al., 2006, 2009b). The majority of sites in our data set (50 of 93) exhibited significant rate increases in the physical removal condition, likely accounting for the directionality of the population effect we observed. However, some of the considered sites also showed significant rate decreases (9 of 93) or were largely unresponsive to target removal (34 of 93). Figure 2D shows the difference in firing rate as well as trial-to-trial variability between visible and invisible conditions (visible - invisible) for individual sites sorted by their physical removal response. 
Consistent with previous literature, sites with negative removal responses (blue) tended to show lower firing rates under perceptual suppression, while most sites with positive removal responses (black) also exhibited higher rates on invisible trials. Interestingly, no such tendency was apparent for trial-to-trial variability, with the majority of sites showing lower Fano factors on invisible trials regardless of their responsiveness. Moreover, we did not observe a significant correlation between the modulation of firing rate and the modulation of trial-to-trial variability considering the absolute values (Pearson's correlation coefficient, $r=-0.13, p=0.22, N=93$ ), suggesting that sites with a greater rate modulation did not necessarily also exhibit a greater modulation of trial-to-trial variability.

A

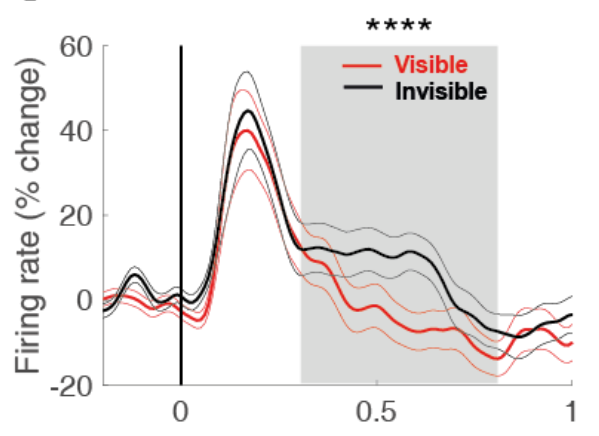

Time from RDM stimulus onset (s)

C

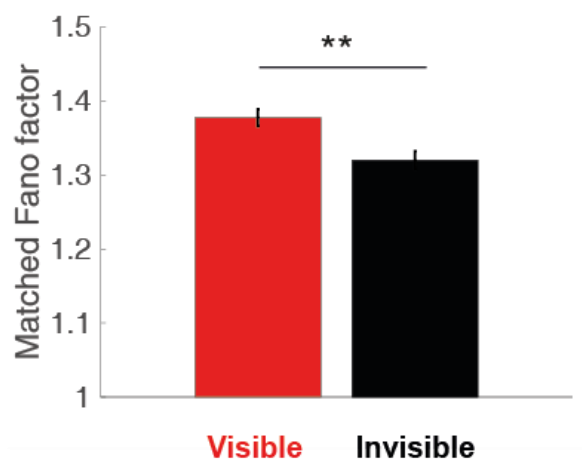

B

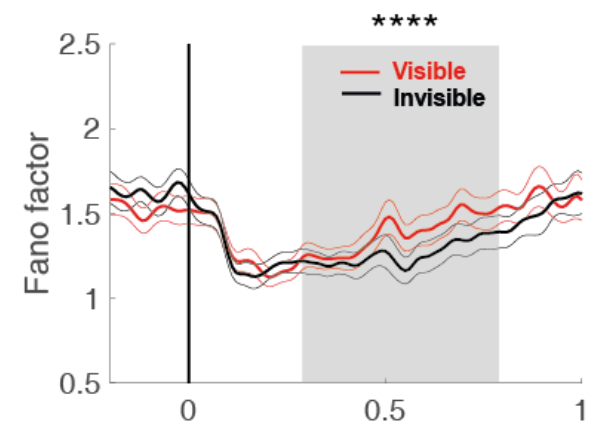

Time from RDM stimulus onset (s)

\section{D}

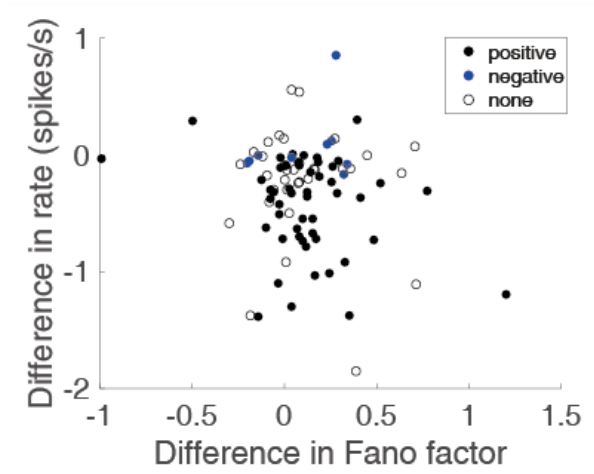

Figure 2. Modulation of mean firing rate and trial-to-trial variability by perceptual suppression. (A) Time courses of percent change in firing rate from baseline for sites with significant rate increases following RDM stimulus onset $(\mathrm{N}=93)$ for ambiguous test trials in which the target remained perceptually visible (red) or became perceptually suppressed ('invisible', black). Error bars indicate +/1 SEM. Gray arrows denote the mean lever release time in 'invisible' trials. (B) Time courses of Fano factor for visible and invisible test trials following RDM onset $(N=93)$. Error bars indicate +/- 1 SEM. (C) Mean Fano factor during the $300-800$ ms post RDM interval +/- 1 SEM for visible and invisible test trials after matching firing rate distributions between the two perceptual outcomes (number of data points: $N=43$ ). Wilcoxon signed-rank tests, ${ }^{* *} p<0.01$. (D) Visible - invisible difference in firing rate (spikes/s) and Fano factor sorted by response to physical removal of the target. Sites with positive (black, $\mathrm{N}=50$ ) or negative (blue, $\mathrm{N}=9$ ) removal responses are represented by filled circles, empty circles indicate sites without significant firing rate changes in response to physical removal $(N=34)$. 
Inspecting the time courses (Figure 2A-B), we noted that the perceptual modulation of trial-to-trial variability appeared to occur considerably later than the firing rate difference. We thus tested the onset of the modulations by perceptual suppression with a $50 \mathrm{~ms}$ sliding window moving in $10 \mathrm{~ms}$ steps that covered the second following RDM stimulus onset. On each consecutive time window, we performed a Wilcoxon signed-rank test between visible and invisible trials in the current window and corrected for multiple comparisons (Bonferroni correction, $p<0.0005$ ). The first significant time window for the modulation of V4 firing rates was $380 \mathrm{~ms}$ after RDM onset, whereas the modulation of trial-to-trial variability first became significant at $520 \mathrm{~ms}$ post RDM onset.

In addition to differences in firing rate, previous studies also observed modulations of low frequency oscillations associated with perceptual suppression, in particular of the alpha and beta band. Similar to spiking variability across trials, low frequency power is reduced during subjective invisibility compared to the stable percept of the target (Wilke et al., 2006). We thus wondered whether the observed trial-totrial variability differences between perceptual states reflected differences in low frequency amplitude and examined the local field potential (LFP) data recorded from the same sites. Figure $3 \mathbf{A}$ shows the Fast Fourier Transform (FFT) of a time window ranging from 500 to 1000 ms post RDM stimulus onset that best captured the observed variability difference between visible and invisible trials, indicating reduced activity during perceptual suppression in a broad range of low frequency oscillations. We then subdivided the low frequency range into the theta $(4-7 \mathrm{~Hz})$, alpha $(8-12 \mathrm{~Hz})$ and beta $(13-20 \mathrm{~Hz})$ physiological frequency bands and computed the envelope of the filtered signal, i.e. the amplitude, for each band separately. The time courses of theta, alpha and beta amplitudes for visible and invisible trials around RDM onset are illustrated in Figure 3B. Estimating the onset of the perceptual modulations of low frequency amplitude in the same manner as for the modulations of spiking activity, we found beta amplitudes to be modulated as early as $280 \mathrm{~ms}$ after RDM stimulus onset, whereas both theta (630 ms after RDM onset) and alpha activity modulations (580 ms after RDM onset) occurred around the same time as the perceptual modulation of trial-to-trial variability.

Comparing average amplitudes in the $500-1000$ ms post RDM interval between perceptual outcomes, we found low frequency activity to be significantly higher during visible than during invisible trials in all considered frequency bands (Wilcoxon signed-rank tests, Bonferroni corrected $p<0.0167$, theta $p=$ 0.002 , alpha $p=9.08 \mathrm{e}-05$, beta $p=1.61 \mathrm{e}-11, \mathrm{~N}=93$ ). We then sought to determine whether low frequency amplitudes and trial-to-trial variability were correlated on a site-to-site basis. 

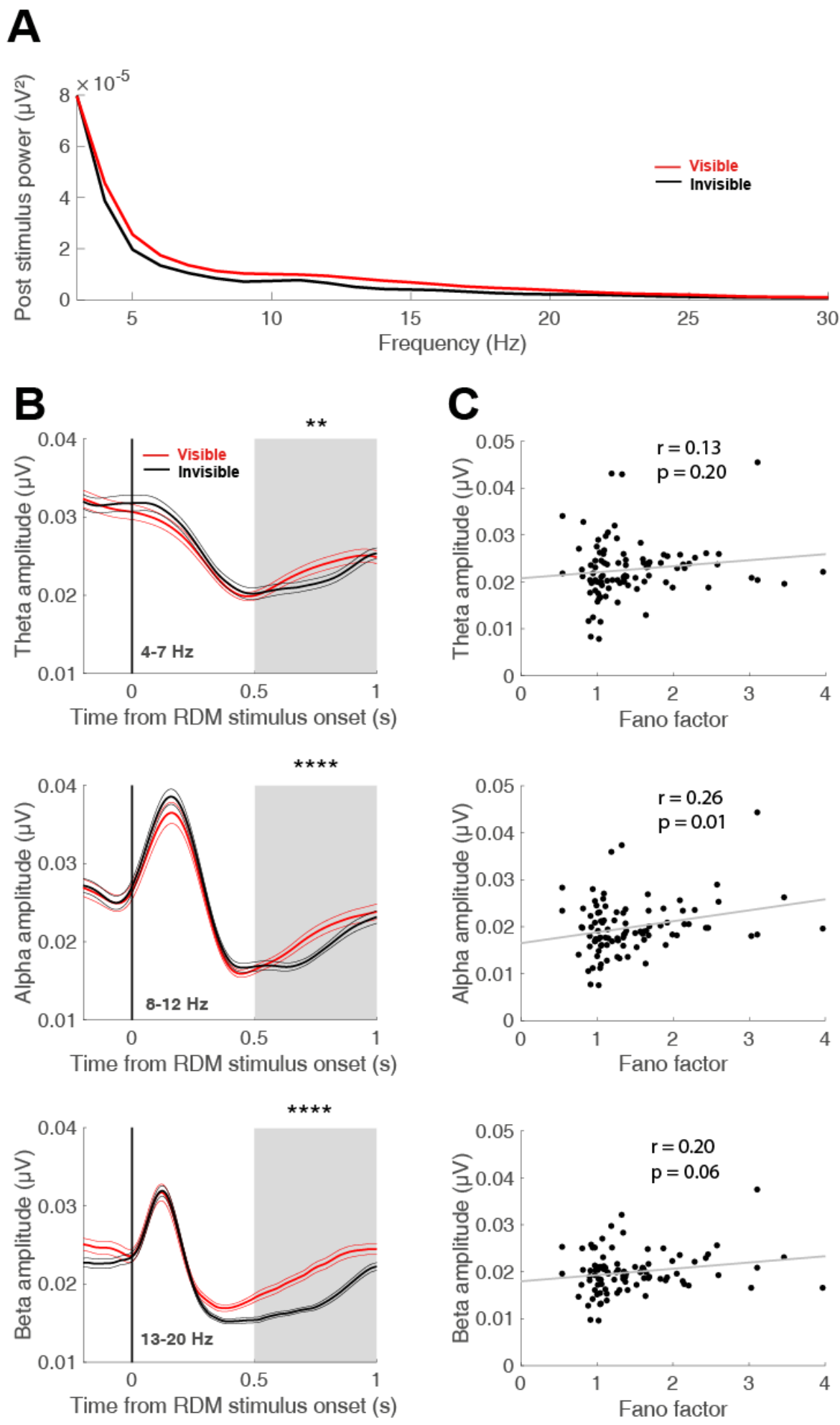

Figure 3. Modulation of low frequency local field potential (LFP) by perceptual suppression and relation to trial-to-trial spiking variability. (A) Fast Fourier Transform (FFT) of the $500-1000$ ms post RDM for visible (red) and invisible (black) trials covering the 3 to $30 \mathrm{~Hz}$ low frequency range. (B) Time courses of theta $(4-7 \mathrm{~Hz})$, alpha $(8-12 \mathrm{~Hz})$ and beta $(13-20 \mathrm{~Hz})$ amplitude around RDM stimulus onset $(\mathrm{N}=93)$ for ambiguous test trials in which the target remained perceptually visible (red) or became perceptually suppressed (black). Wilcoxon signed-rank tests, ${ }^{* *} p<0.01,{ }^{* * * *} p<0.0001$. Error bars indicate +/- 1 SEM. (C) Correlation between average theta, alpha and beta amplitude and Fano factor in the $500-1000$ ms post RDM stimulus window across ambiguous test trials. Pearson's correlation coefficient and corresponding $p$ value. 
We found alpha amplitude and Fano factors across ambiguous test trials regardless of perceptual outcome to be positively correlated (Pearson's correlation coefficient, $r=0.26, p=0.01, N=93$, Bonferroni corrected $p<0.0167$, Figure 3C), suggesting that sites with larger alpha amplitudes also exhibited greater spiking variability across trials. We did not observe a significant correlation between beta amplitude and trial-to-trial variability (Pearson's correlation coefficient, $r=0.20, p=0.06, N=93$, Bonferroni corrected $p<0.0167)$ or between theta amplitude and trial-to-trial spiking variability (Pearson's correlation coefficient, $r=0.16, p=0.15, N=93$, Bonferroni corrected $p<0.0167$ ).

\subsection{Discussion}

In the GFS condition that probabilistically induced perceptual suppression, target stimuli reported as disappearing were associated with reduced trial-to-trial variability in area V4. Importantly, this difference was also present when we equated firing rate distributions between visible and invisible trials using a mean matching procedure, suggesting that the variability modulation occurred independently of the rate difference between perceptual conditions. This interpretation is further supported by the observations that the effect directionality did not differ based on the directionality of the physical removal response, that the perceptual modulations of variability and firing rate were uncorrelated, and that the modulation of trial-to-trial variability occurred considerably later than the difference in firing rates. Instead, we consider it highly likely that the reduced spiking variability under perceptual suppression reflects concurrent reductions in low frequency power. Amplitudes of the theta, alpha and beta frequency bands significantly differentiated between perceptual outcomes in a time window coinciding with the trial-totrial variability difference we observed, and we found spiking variability and alpha amplitude to be positively correlated on a site-to-site basis. While we only observed a clear correlation between trial-totrial variability and alpha activity, it seems probable that this relationship more broadly extends to neighboring physiological frequency bands. Interestingly, the onset of both the perceptual modulation of alpha and theta amplitude coincided with the late onset of the spiking variability modulation, while beta amplitudes differed between visibility conditions at a notably earlier time when the target first became subjectively invisible.

One of the greatest challenges in consciousness research is to determine whether neural signatures that have been found to correlate with stimulus visibility are in fact specific to visual awareness or 
instead reflect other confounding factors such as attention, perceptual decision-making or motor planning associated with reporting about subjective percepts (Tsuchiya et al., 2015). In particular, the modulation of low frequency power by perceptual suppression in the visual thalamus has been shown to be absent under passive viewing conditions when monkeys were not required to report their percept, although it has to be noted that the comparison was made between unambiguous stimulation conditions that were assumed to result in either subjective disappearances or a stable perception of the target (Wilke et al., 2009b). In our case, the onset of the variability modulation as well as the onset of the modulation of alpha and theta amplitude occurred at roughly the same time as the average perceptual response around $500 \mathrm{~ms}$ after RDM stimulus onset, which was further only present in the invisible condition. We thus cannot exclude that these effects are related to the decision to report the target disappearance or even the motor response itself. Alternatively, it is possible that the subjective disappearance of the target or its reappearance after several hundred milliseconds of perceptual suppression, possibly even brought about by the act of reporting, may draw subjects' attention.

Based on the latency of the variability and theta / alpha amplitude effects we do not interpret them to reflect the cause of target invisibility as we estimated target disappearances to occur on average around 200 ms after RDM onset and would thus have expected neural signatures of target suppression to be discernible at this stage. To some degree this argument also applies to the hypothesis that the effects may reflect the steady state of perceptual suppression, but it is theoretically possible that differences in low frequency amplitude could only arise after the initial synchronization of low frequency activity by the onset of the RDM stimulus, whereby subsequent higher amplitudes result in higher variability across trials. On invisible trials, stimulus presentation was not interrupted for additional $1000 \mathrm{~ms}$ after the monkey had indicated the disappearance of the target by a lever release, so it appears at least possible that the reduced low frequency activity may indicate maintained target suppression. The modulations of trial-to-trial variability as well as firing rates by perceptual suppression we observed bear a striking resemblance to the effects of spatial attention on spiking responses previously reported in area V4 (Mitchell et al., 2007, 2009; Cohen and Maunsell, 2009). Moreover, several psychophysical studies using related perceptual suppression paradigms such as motion-induced blindness (MIB) have consistently shown that directing attention to a target stimulus increases the probability of its subjective disappearance (Geng et al., 2007; Carter et al., 2008; Schölvinck and Rees, 2010). It is thus conceivable that the decreased spiking variability on invisible trials as well as the higher firing rates 
during the sustained response might reflect increased anticipatory attention towards the target stimulus which in turn facilitates its perceptual suppression. In principle, this interpretation would also be consistent with the absence of significant effects under no report conditions when the monkeys are not or only minimally engaged in the perceptual task and trials are not sorted by their perceptual outcomes determined by spontaneous fluctuations in attentional state. In summary, we can conclude that trial-totrial spiking variability varies with subjective awareness during perceptual suppression, but further research is needed to disentangle its role in conscious perception from other possible causes by using similar response requirements in the compared perceptual conditions, employing no-report paradigms and varying task engagement and/or difficulty, which we did not do in the current study. Moreover we consider it likely that the observed differences in trial-to-trial variability result from concurrent differences in low frequency amplitude, especially but likely not exclusively in the alpha band, which warrants further studies and theoretical modeling approaches investigating the relationship between oscillatory brain activity and neural variability in more detail.

\subsection{References}

Arazi A, Censor N, Dinstein I (2017) Neural Variability Quenching Predicts Individual Perceptual Abilities. The Journal of Neuroscience 37:97-109.

Britten KH, Shadlen MN, Newsome WT, Movshon JA (1992) The analysis of visual motion: a comparison of neuronal and psychophysical performance. J Neurosci 12:4745-4765.

Carter O, Luedeman R, Mitroff S, Nakayama K (2008) Motion induced blindness: The more you attend the less you see. Journal of Vision 8:237-237.

Churchland MM et al. (2010) Stimulus onset quenches neural variability: a widespread cortical phenomenon. Nature Neuroscience 13:369-378.

Cohen MR, Maunsell JHR (2009) Attention improves performance primarily by reducing interneuronal correlations. Nature Neuroscience 12:1594-1600.

Geng H, Song Q, Li Y, Xu S, Zhu Y (2007) Attentional modulation of motion-induced blindness. Chin Sci Bull 52:1063-1070.

Ledberg A, Montagnini A, Coppola R, Bressler SL (2012) Reduced Variability of Ongoing and Evoked Cortical Activity Leads to Improved Behavioral Performance. PLoS One 7 Available at: https://www.ncbi.nlm.nih.gov/pmc/articles/PMC3427304/ [Accessed March 5, 2020].

Maier A, Wilke M, Aura C, Zhu C, Ye FQ, Leopold DA (2008) Divergence of fMRI and neural signals in V1 during perceptual suppression in the awake monkey. Nature Neuroscience 11:1193-1200.

Mitchell JF, Sundberg KA, Reynolds JH (2007) Differential attention-dependent response modulation across cell classes in macaque visual area V4. Neuron 55:131-141. 
Mitchell JF, Sundberg KA, Reynolds JH (2009) Spatial Attention Decorrelates Intrinsic Activity Fluctuations in Macaque Area V4. Neuron 63:879-888.

Ni AM, Ruff DA, Alberts JJ, Symmonds J, Cohen MR (2018) Learning and attention reveal a general relationship between population activity and behavior. Science 359:463-465.

Parker AJ, Newsome WT (1998) SENSE AND THE SINGLE NEURON: Probing the Physiology of Perception. Annual Review of Neuroscience 21:227-277.

Scaglione A, Moxon KA, Aguilar J, Foffani G (2011) Trial-to-trial variability in the responses of neurons carries information about stimulus location in the rat whisker thalamus. Proceedings of the National Academy of Sciences 108:14956-14961.

Schölvinck ML, Rees G (2010) Neural Correlates of Motion-induced Blindness in the Human Brain. Journal of Cognitive Neuroscience 22:1235-1243.

Schurger A, Pereira F, Treisman A, Cohen JD (2010a) Reproducibility Distinguishes Conscious from Nonconscious Neural Representations. Science 327:97-99.

Schurger A, Pereira F, Treisman A, Cohen JD (2010b) Reproducibility Distinguishes Conscious from Nonconscious Neural Representations. Science 327:97-99.

Schurger A, Sarigiannidis I, Naccache L, Sitt JD, Dehaene S (2015a) Cortical activity is more stable when sensory stimuli are consciously perceived. PNAS 112:E2083-E2092.

Schurger A, Sarigiannidis I, Naccache L, Sitt JD, Dehaene S (2015b) Cortical activity is more stable when sensory stimuli are consciously perceived. Proceedings of the National Academy of Sciences 112:E2083-E2092.

Shadlen MN, Britten KH, Newsome WT, Movshon JA (1996) A computational analysis of the relationship between neuronal and behavioural responses to visual motion. J Neurosci 16:1486-1510.

Shadlen MN, Newsome WT (1998) The Variable Discharge of Cortical Neurons: Implications for Connectivity, Computation, and Information Coding. J Neurosci 18:3870-3896.

Softky WR, Koch C (1993) The highly irregular firing of cortical cells is inconsistent with temporal integration of random EPSPs. J Neurosci 13:334-350.

Stein RB, Gossen ER, Jones KE (2005) Neuronal variability: noise or part of the signal? Nat Rev Neurosci 6:389-397.

Tsuchiya N, Wilke M, Frässle S, Lamme VAF (2015) No-Report Paradigms: Extracting the True Neural Correlates of Consciousness. Trends in Cognitive Sciences 19:757-770.

Wilke M, Logothetis NK, Leopold DA (2006) Local field potential reflects perceptual suppression in monkey visual cortex. PNAS 103:17507-17512.

Wilke M, Mueller K-M, Leopold DA (2009a) Neural activity in the visual thalamus reflects perceptual suppression. PNAS 106:9465-9470.

Wilke M, Mueller K-M, Leopold DA (2009b) Neural activity in the visual thalamus reflects perceptual suppression. Proceedings of the National Academy of Sciences 106:9465-9470.

Zohary E, Shadlen MN, Newsome WT (1994) Correlated neuronal discharge rate and its implications for psychophysical performance. Nature 370:140-143. 


\section{Chapter IV}

\section{Reduced alpha amplitudes predict perceptual suppression}

Eva Poland ${ }^{1}$, Aishwarya Bhonsle ${ }^{1}$, Iris Steinmann ${ }^{1}$ and Melanie Wilke ${ }^{1,2,3}$

${ }^{1}$ Department of Cognitive Neurology, University of Goettingen, Robert-Koch-Str. 40, Goettingen, 37075, Germany

${ }^{2}$ German Primate Center, Leibniz Institute for Primate Research, Kellnerweg 4, Goettingen, 37077, Germany

${ }^{3}$ Leibnitz Science Campus Primate Cognition, Kellnerweg 4, Goettingen, 37077, Germany

\section{Author contributions}

M. Wilke and E. Poland developed the study concept and task and A. Bhonsle and E. Poland recorded the data. E. Poland analyzed the data. I. Steinmann, A. Bhonsle and E. Poland interpreted the data and contributed to the analysis. M. Wilke and E. Poland wrote the manuscript, all authors provided critical revisions to the manuscript and approved the final version of the manuscript. 


\subsection{Abstract}

The amplitude of prestimulus alpha oscillations over parieto-occipital cortex has been shown to predict visual detection performance of masked and threshold-level stimuli. Whether alpha activity similarly predicts target visibility during perceptual suppression, another type of illusion commonly used to investigate visual awareness, is presently unclear. Here, we examined prestimulus alpha activity in the electroencephalogram (EEG) of healthy participants recorded in the context of a generalized flash suppression (GFS) paradigm during which salient target stimuli are rendered subjectively invisible on a subset of trials following the onset of a full-field motion stimulus. To determine whether modulations were hemisphere-specific, we presented target stimuli in the left and right visual hemifield. Unlike for masking or threshold paradigms, alpha $(8-12 \mathrm{~Hz})$ amplitude prior to motion onset was significantly higher on trials on which the targets remained subjectively visible compared to trials during which the targets were perceptually suppressed. Further, individual prestimulus alpha amplitudes strongly correlated with the individual degree of trial-to-trial variability quenching following stimulus onset, a measure that has been shown to similarly affect subsequent perceptual performance. Our results indicate that that variability quenching in visual cortex is closely linked to prestimulus alpha activity, and that predictive correlates of conscious perception derived from perceptual suppression paradigms differ substantially from those of masking and threshold paradigms, likely reflecting the effectiveness of the suppressor stimulus.

\subsection{Introduction}

Conscious perception is a constructive process which relies not only on physical input but also on the internal state of the brain. This is evidenced by neurophysiological studies that employed perceptual suppression paradigms such as flash suppression or motion-induced blindness (MIB), wherein salient target stimuli are intermittently erased from awareness despite identical physical stimulation (Kim and Blake, 2005; Storm et al., 2017). The contribution of alpha activity to subjective visibility has sparked a particular discussion because it has been shown to be reduced prior to perceived compared to nonperceived stimuli for masking paradigms and paradigms presenting stimuli at the perceptual threshold (Ergenoglu et al., 2004; Hanslmayr et al., 2007; Dijk et al., 2008; Romei et al., 2010; Benwell et al., 
2017). On the other hand, previous studies employing perceptual suppression observed lower alpha power during target invisibility following the onset of the suppressing motion stimulus (Wilke et al., 2006; Maier et al., 2008; Vidal et al., 2015), which is diminished in the absence of active report (Wilke et al., 2009; Keliris et al., 2010). Whether perceptual suppression is affected by ongoing alpha activity is presently unclear.

In addition to predicting the detection of masked and threshold-level stimuli, lower average prestimulus alpha amplitudes were observed in individuals with a higher performance in detecting masked stimuli compared to individuals who failed to perceive them (Hanslmayr et al., 2007). A recent study asked whether prestimulus alpha activity primarily influences subjective awareness or objective perceptual accuracy by combining a contrast judgement of a briefly presented stimulus with ratings of subjective awareness and found that alpha power prior to stimulus presentation correlated with visual awareness but not objective performance (Benwell et al., 2017). Moreover, alpha activity has also been associated with visuospatial attention. Studies using a cueing task to direct spatial attention to stimuli presented in the left or right visual hemifield consistently found a lateralization of alpha activity, with lower parietooccipital alpha amplitudes over the hemisphere contralateral to the attended stimulus location (Worden et al., 2000; Sauseng et al., 2005; Kelly et al., 2006; Thut, 2006; Mo et al., 2011). Alpha activity has thus been implicated as an inhibitory mechanism of the brain (Klimesch et al., 2007), suppressing information that is not behaviourally relevant (Foxe and Snyder, 2011). A recent study showed that alpha power modulations reflect the suppression of distractors independent of target selection (Wöstmann et al., 2019). Reduced alpha amplitudes have also been suggested to primarily reflect increased cortical excitability (Romei et al., 2008; lemi et al., 2017) rather than increased perceptual acuity (Lange et al., 2013; Samaha et al., 2017). Posterior alpha power has further been linked to trialto-trial variations in attentional state, with higher levels of subjectively reported attentiveness accompanying reduced alpha activity (Macdonald et al., 2011).

Another measure closely linked to both visual perception and attention is the variability of neural signals across trials (Dinstein et al., 2015). Neural activity exhibits a large degree of trial-to-trial variance that has been attributed to slow fluctuations in cortical excitability that are larger in synchronized states and involve the majority of neurons in visual cortex (Arieli et al., 1996; Mitchell et al., 2009; Schölvinck et al., 2015). Upon stimulus onset trial-to-trial variability substantially decreases in a wide range of cortical 
areas (Churchland et al., 2010), an effect referred to as variability quenching. The individual magnitude of variability quenching observed in the human EEG has been shown to be a reproducible characteristic predicting lower perceptual discrimination thresholds in subjects who quench more (Arazi et al., 2017b, 2017a). Similar to alpha amplitude, trial-to-trial variability is lower for attended compared to unattended stimuli (Mitchell et al., 2007, 2009; Cohen and Maunsell, 2009), and reduced trial-to-trial variability predicts visual detection of stimuli presented at the perceptual threshold (Ress et al., 2000; Schurger et al., 2015). Despite these functional similarities, trial-to-trial variability and alpha amplitude have been discussed in separate bodies of literature and only very recently, variability quenching has been related to the decrease in alpha/beta amplitude with stimulus onset (Daniel et al., 2019). Here, we test the hypothesis that the magnitude of the respective decrease may be dependent on the amplitude of ongoing alpha activity prior to stimulus onset, which has been shown to predict subsequent perception.

In the current study, we evaluate whether alpha amplitudes prior to the onset of the motion stimulus predict subsequent subjective target disappearances in perceptual suppression. Comparing individual levels of the two measures between subjects, we further investigate the relationship between prestimulus alpha activity and variability quenching.

\subsection{Methods}

\section{Subjects}

A total of 35 healthy subjects participated in the current study. Of those, 4 subjects were excluded from the analysis due to general exclusion criteria (one due to red-green colour blindness, one due to the subject reporting no subjective disappearances under generalized flash suppression (GFS), one due to $<75 \%$ accuracy in the physical removal condition and one due to technical error with the EEG recording setup). For the analysis of general visibility effects we compared trials in which both targets had disappeared ('invisible') and trials during which neither target disappeared ('visible'). We required a minimum of 20 trials in each perceptual condition, resulting in a cohort of 27 subjects (12 male / 15 female, 14 left handers / 13 right handers, between 18 and 50 years of age). The mean number of visible trials was 159 +/- SD 133, the mean number of invisible trials was 215 +/- SD 146. All subjects gave their informed consent to and were rewarded for their participation in the study. 


\section{Experimental procedure}

Data were recorded in a single session lasting 3 to 4 hours in total, including EEG preparation, experiment and breaks. To exclude colour blindness subjects judged a set of 20 Ishihara plates of which 19 correct identifications were required for inclusion in the study (Clark, 1924). Prior to the experiment subjects performed 10 to 20 practice trials in order to familiarize themselves with the generalized flash suppression (GFS) task (Wilke et al., 2003). We carefully instructed subjects to indicate target disappearances based on whether the target stimuli had disappeared completely, regardless whether perceptual suppression persisted until the end of the trial. Following EEG preparation, subjects completed 6 experimental blocks, each lasting approximately 10 minutes. Subjects were seated in front of a $60 \times 34 \mathrm{~cm}$ computer screen and placed their head on a chin rest with an eye to screen distance of $70 \mathrm{~cm}$. During the experiment, lights in the recording room were turned off and additional curtains were used to screen the subject from extraneous light. Between blocks subjects had the opportunity to take brief breaks. Stereoscopy was achieved using anaglyphic glasses. To control for individual differences in eye dominance, the positions of the red and green filters were interchanged with each configuration being used for 3 of the 6 experimental blocks in varied order to equate target presentations in the dominant and non-dominant eyes. All experimental procedures were approved by the ethics committee of the University Medicine Göttingen (UMG, Germany).

\section{Stimuli and task}

Stimuli were programmed and presented in Matlab 2015b (The MathWorks Inc., Natick MA, USA) using Psychtoolbox-3 (Kleiner, n.d.; Brainard, 1997). Each trial began with central fixation for 1 second (Figure 1A). Subjects were then presented with two salient red target stimuli (size $3^{\circ}$ of visual angle) in the left and right visual hemifield $\left(7^{\circ}\right.$ of visual angle horizontal distance from centre, $3^{\circ}$ of visual angle vertical distance from centre). The targets were only presented to one eye by means of anaglyphic redgreen glasses. After 2 seconds, a random dot motion (RDM) pattern consisting of 2000 green dots moving at a speed of $10 \%$ second was shown to the respective other eye for 2 seconds, which resulted in the subjective disappearance of one or both target stimuli in a subset of trials. Following the end of stimulus presentation, subjects were prompted to report their perception, that is the subjective disappearance of both, neither, the right or the left target stimulus, with a mouse click using their 
preferred hand equivalent to their handedness. In addition to the experimental trials (600 trials), each subject performed 120 control trials intermixed with the experimental trials in which either the left or the right target stimulus was physically removed following the onset of the RDM pattern.

\section{EEG acquisition and preprocessing}

EEG activity was recorded from 63 electrodes without ECG signal (64-Channel Standard BrainCap, Brain Products, Gilching, Germany) using BrainVision Recorder (Brain Products, Gilching, Germany). Electrode impedances were kept below $20 \mathrm{k} \Omega$ throughout the experiment. EEG data were preprocessed and analysed using the Fieldtrip toolbox (Oostenveld et al., 2011) and custom-written software in Matlab 2015b (The MathWorks Inc., Natick MA, USA). The data were recorded at a sampling rate of $1000 \mathrm{~Hz}$ and bandpass filtered between $0.1-250 \mathrm{~Hz}$. Trials containing muscle artefacts, jumps or clipping artefacts were identified automatically and rejected following visual inspection. An independent component analysis (ICA) was performed to identify eye movement artefacts and eye movement related components were removed. The data were then re-referenced to a common reference.

\section{Prestimulus alpha amplitude}

For the analysis of alpha amplitudes, the data of the parieto-occipital electrodes $\mathrm{O} 1, \mathrm{O} 2, \mathrm{Oz}, \mathrm{PO}, \mathrm{PO}$, PO4, PO7 and PO8 were bandpass filtered at $8-12 \mathrm{~Hz}$ with a $4^{\text {th }}$ order Butterworth filter and subsequently Hilbert transformed. We then considered the absolute values of the Hilbert transform, equivalent to the envelope of the filtered signal, in a time window spanning the second prior to the onset of the RDM stimulus (prestimulus window) pooled over the parieto-occipital electrodes for statistical analysis of prestimulus alpha amplitude. The selected electrodes cover occipital cortex and were chosen based on our expectation of possible modulations occurring in the visual system, as well as for consistency with previous research having investigated prestimulus alpha power within the same set of electrodes (Hanslmayr et al., 2007). For the main comparison between target visibility outcomes, we considered trials in which subjects had reported both targets to disappear (invisible) and trials in which both targets had been reported to remain visible (visible). In order to determine the subjects' individual alpha frequency (IAF), we additionally calculated the Fast Fourier Transform (FFT) of the prestimulus 
window for all parieto-occipital electrodes across perceptual outcomes between 1 and $30 \mathrm{~Hz}$ at a resolution of $1 \mathrm{~Hz}$ and identified the peak frequency between 5 and $15 \mathrm{~Hz}$ in each subject. We then calculated equivalent FFTs for visible and invisible trials separately and compared prestimulus FFT power at the IAF that was determined for each subject between perceptual outcomes. For topographical representation we calculated the average absolute value of the $8-12 \mathrm{~Hz}$ filtered Hilbert transform in the second prior to RDM onset for each channel separately. The difference between visible and invisible conditions was then calculated by subtracting the average prestimulus alpha amplitude of invisible trials from the mean prestimulus alpha amplitude of visible trials in each subject. For the comparison of alpha amplitudes in the left and right hemisphere we omitted the central electrodes $\mathrm{Oz}$ and $\mathrm{POz}$ of the initial selection and considered the lateral electrodes $\mathrm{O} 1, \mathrm{PO} 3$ and $\mathrm{PO} 7$ as left and $\mathrm{O} 2, \mathrm{PO} 4$ and $\mathrm{PO} 8$ as right hemispheric ROls respectively. For the comparison of prestimulus alpha amplitude to stimulus-induced changes in trial-to-trial variability we computed the average prestimulus alpha amplitude per subject over all experimental trials regardless of their perceptual outcome. Due to the fact that most variables were not normally distributed, we applied non-parametric statistical methods throughout analyses and Wilcoxon signed-rank tests were used for within-subject comparisons.

\section{Trial-to-trial variability}

In order to assess variability across trials, we first calculated the variance across trials for the signal of the same parieto-occipital electrodes $\mathrm{O} 1, \mathrm{O} 2, \mathrm{Oz}, \mathrm{POz}, \mathrm{PO}, \mathrm{PO} 4, \mathrm{PO} 7$ and $\mathrm{PO} 8$ for each $1 \mathrm{~ms}$ time point of each trial across all experimental trials for each subject. We then computed the relative variance as the percent change in variance with stimulus onset from a baseline 500 to $0 \mathrm{~ms}$ prior to the onset of the RDM stimulus. The degree of variability quenching for individual subjects was then determined as the average relative variance in a time window $500 \mathrm{~ms}$ to $1500 \mathrm{~ms}$ post RDM stimulus which best covered the variance decline (poststimulus window). We calculated the non-parametric Spearman's rank correlation coefficient between the individual degree of variability quenching and the individual decrease in alpha amplitude based on the same baseline and post RDM stimulus window. Similarly, we calculated the Spearman's rank correlation between the individual magnitude of prestimulus alpha amplitude across all experimental trials and the individual degree of variability quenching as well as the decrease in alpha amplitude with stimulus onset in order to determine whether the decreases were 
linked to prestimulus activity. To assess the perceptual relevance of stimulus-induced decreases of trialto-trial variability we compared its change with motion onset using the same previously used baseline (-500 - $0 \mathrm{~ms})$ and poststimulus window (500 - $1500 \mathrm{~ms}$ ) between visible and invisible trials. For illustration of individual time courses of alpha amplitude and trial-to-trial variance, we z-scored both measures by subtracting the mean and dividing by the standard deviation according to $z=(x-\mu) / \sigma$.

\subsection{Results}

We recorded 600 experimental trials from each subject performing a generalized flash suppression (GFS) task (Wilke et al., 2003), a visual illusion by which salient target stimuli can be rendered subjectively invisible after the onset of a random dot motion (RDM) stimulus (Figure 1A). Following central fixation, subjects were presented with two target stimuli consisting of red disks in the left and right visual hemifield for 2 seconds. Then, the RDM stimulus was additionally presented for 2 seconds. After the end of stimulus presentation, subjects were asked to report whether the right, the left, both or neither target had perceptually disappeared. Perceptual outcomes are summarized in Figure 1B. Generally stimuli were more often suppressed or remained subjectively visible together rather than disappearing individually, although unilateral disappearances of only the left or the right target occurred (left target: $0.12+/$ SD 0.9 , right target: $0.13+/$ SD 0.7 , both targets: $0.42+/-$ SD 0.26 , neither target: $0.33+/-$ SD 0.27 ). As a control, each session further included a total of 120 catch trials (60 for the right and left target respectively) during which either the right or the left target stimulus was physically removed from screen. Accuracy in detecting these physical removals was very high in all subjects $(98 \%$ $+/$ - SD 3\% for left target removals and $99 \%+/$ - SD $2 \%$ for right target removals, Figure 1C), suggesting that subjects performed the task correctly and were attentive throughout the experimental session.

We then evaluated whether parieto-occipital prestimulus alpha activity reflected the subjective visibility of the targets. The time course of mean alpha amplitude across subjects around the time of the onset of the RDM stimulus for trials in which both targets had disappeared (invisible) and trials in which neither target had disappeared (visible) is shown in Figure 2A. Due to the timing of GFS, we considered a time window prior to the onset of the motion stimulus as the prestimulus interval rather than a time window preceding the target as used in previous studies employing threshold-level or masking paradigms to 
contrast subjective visibility states. Comparing the average alpha amplitude of all parieto-occipital electrodes in the second preceding the onset of the RDM stimulus, we observed a significant difference between trials during which the targets were suppressed and trials during which they remained visible (Wilcoxon signed-rank test, $\mathrm{p}=0.0039, \mathrm{~N}=27$, mean alpha amplitude visible $4.41+/$ - SD 2.36, mean alpha amplitude invisible $4.23+/-$ SD 2.56). We did not observe any differences between perceptual conditions in alpha amplitude post RDM stimulus (poststimulus window, Wilcoxon signed-rank test, $p=$ 0.94).

A
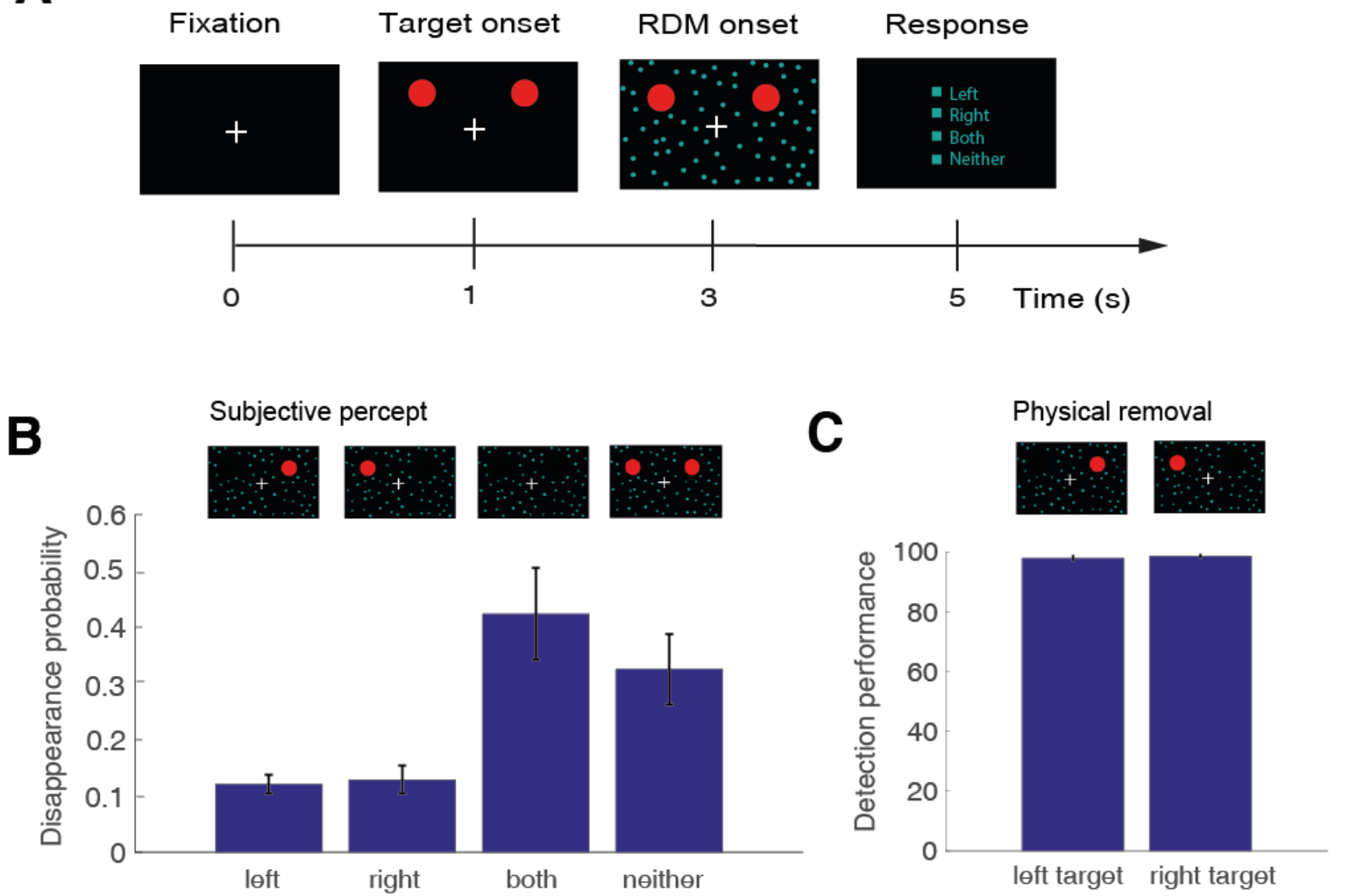

Figure 1. Generalized flash suppression (GFS) paradigm and perceptual outcomes. A) Each experimental trial began with central fixation, followed by the onset of two salient red target stimuli in the upper left and right visual hemifield. After the target had been presented for 2 seconds, a random dot motion (RDM) stimulus consisting of green dots was shown, resulting in the disappearance of one or both target stimuli in a subset of trials. After each trial, subjects reported whether the left, the right, both or neither target had disappeared by selecting one of the options with a mouse click. Stereoscopy was achieved using red-green anaglyphic glasses. B) Probabilities of the left, the right, both or neither target disappearing for all 27 subjects. Corresponding subjective percepts are illustrated above the respective bars. Error bars indicate +/- 1 SEM across subjects. C) Percentage of correct reports of physical removal of the left or the right target during the control conditions. Error bars indicate $+/-1$ SEM across subjects. 
The visible - invisible difference in prestimulus alpha amplitude was positive for almost all subjects regardless of the mean alpha amplitude across perceptual conditions, suggesting that prestimulus alpha amplitudes were consistently lower on trials during which the targets were perceptually suppressed independent of the individual mean amplitude (Figure 2B). Figure 2C shows the FFT of the second prior to RDM stimulus onset across subjects for visible and invisible trials. The mean individual alpha frequency (IAF) across subjects was $9.89 \mathrm{~Hz}+/-$ SD 1.37, ranging from 7 to $13 \mathrm{~Hz}$. Comparing power specifically at the subjects IAF, we found IAF power to be significantly higher on visible compared to invisible trials (Wilcoxon signed-rank test, $\mathrm{p}=0.008, \mathrm{~N}=27$ ), indicating that the effect was in fact due to an amplitude difference. We further determined the individual peak alpha frequency during the second prior to RDM onset for visible and invisible trials separately and found them to not statistically differ (visible $9.85 \mathrm{~Hz}+/-$ SD 1.32, invisible $9.92 \mathrm{~Hz}+/-\mathrm{SD} 1.36$, Wilcoxon signed-rank test $p=0.69, \mathrm{~N}=27$ ). The topography of the visible - invisible difference in alpha amplitude across all 63 electrodes (Figure 2D) suggested that the effect was most prominent in right parietooccipital cortex. Prestimulus alpha amplitudes were generally larger in the right than in the left parietooccipital electrodes (Wilcoxon signed-rank test, $\mathrm{p}=0.02, \mathrm{~N}=27$ ) regardless of perceptual outcome. Comparing alpha amplitudes during the second preceding RDM onset between visible and invisible conditions in each hemisphere separately (Figure 2E), we found that both hemispheres contributed to the effect (Wilcoxon signed-rank tests, left hemisphere $p=0.007$, right hemisphere $p=0.002, N=27$ ). Although previously discussed in separate bodies of literature, findings characterizing the role of prestimulus alpha activity in visual perception bear many similarities to those reported for neural variability across trials. In particular, the magnitude of the variability reduction upon stimulus onset has previously been shown to be a reproducible trait closely linked to individual perceptual abilities (Arazi et al., 2017a), and very recently, to reflect stimulus-induced decreases in alpha power (Daniel et al., 2019). Inspecting time courses of trial-to-trial variance and alpha amplitudes of individual subjects, we noted that both measures closely co-varied (Figure 3A). As expected from previous studies (Churchland et al., 2010; Arazi et al., 2017b, 2017a; Poland et al., 2019), variability across trials significantly decreased with the onset of the RDM stimulus in parieto-occipital cortex (relative variance, Wilcoxon signed-rank test, $\mathrm{p}=0.00009, \mathrm{~N}=27$ ). Likely due to the ongoing visual stimulation by the dynamic RDM pattern, trial-to-trial variability remained at a constant lower level during the 2 second stimulus presentation interval. 
Subjects thereby notably differed in the degree of variability quenching defined as the mean relative variance in a stable interval $500-1500$ ms post stimulus capturing the variance decrease (Figure 3B).

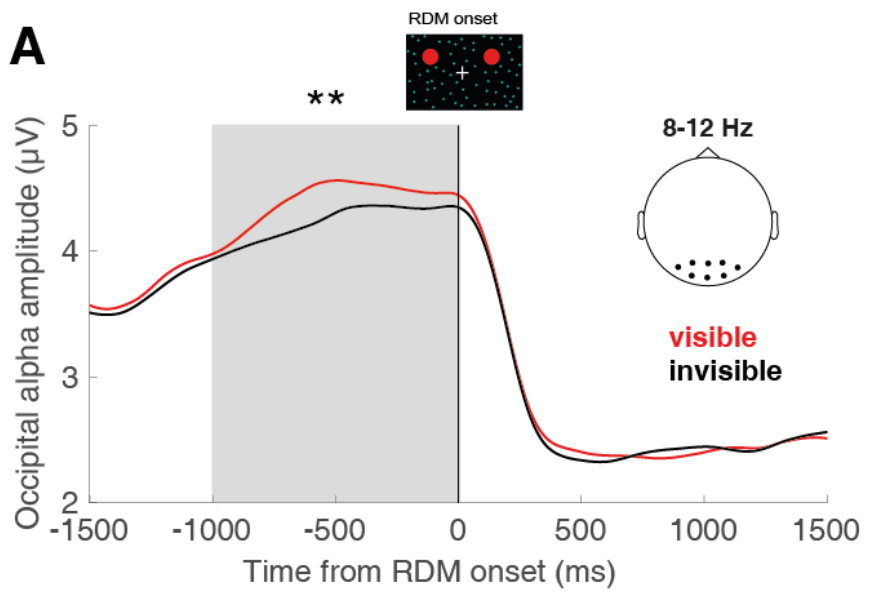

B Difference (visible - invisible)

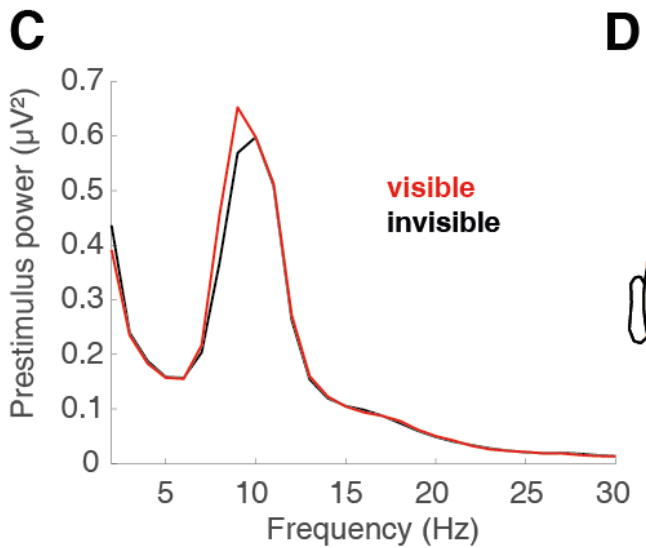

D Difference (visible - invisible)
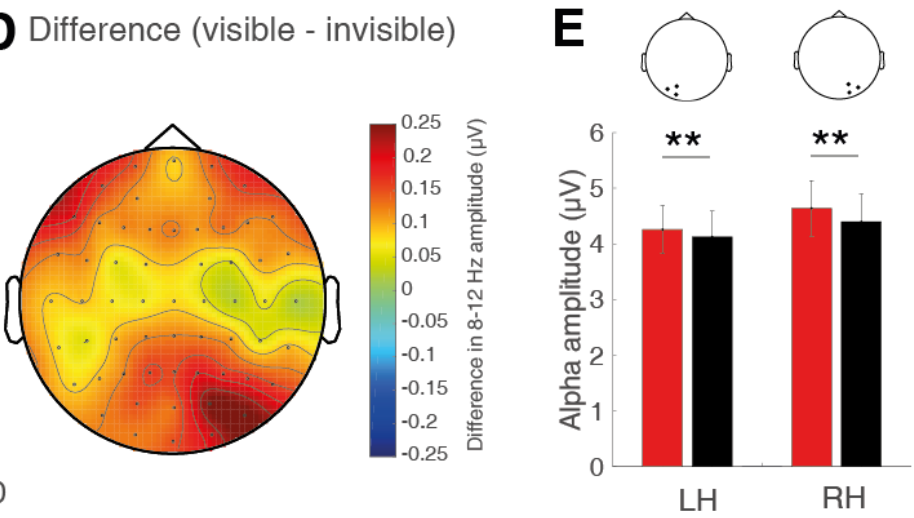

Figure 2. Prestimulus alpha amplitude predicts perceptual suppression. A) Time course of alpha band $(8-12 \mathrm{~Hz})$ amplitudes for trials in which both targets remained subjectively visible (red, 'neither' condition) and trials in which both targets were perceptually suppressed (black, 'both' condition, $\mathrm{N}=$ 27). The zero mark denotes the onset of the RDM stimulus following which the targets were perceptually suppressed. Data represent the mean of all parietal-occipital electrodes. ${ }^{* *}$ denotes a significant group difference in alpha amplitude as assessed by Wilcoxon signed-rank test $(p<0.01)$. B) Difference in parieto-occipital alpha $(8-12 \mathrm{~Hz})$ amplitude between visible and invisible conditions (visible - invisible) in the second preceding RDM onset for individual subjects as a function of the individual average 8-12 $\mathrm{Hz}$ amplitude in the second preceding RDM onset across perceptual conditions, outlier corrected > 2SD, $N=26$. C) Fast Fourier Transform (FFT) of the second preceding the RDM stimulus for visible and invisible trials across subjects. D) Topography of the visible - invisible difference in $8-12 \mathrm{~Hz}$ amplitude in the second prior to RDM onset, $\mathrm{N}=27$. E) Average alpha amplitudes during the second preceding RDM onset for visible (red) and invisible (black) trials for the left ( $\mathrm{LH}$, electrodes O1, PO3, PO7) and right hemisphere ( $\mathrm{RH}$, electrodes $\mathrm{O} 2, \mathrm{PO} 4, \mathrm{PO} 8)$ separately. ${ }^{* *}$ denotes a significant group difference in alpha amplitude as assessed by Wilcoxon signed-rank test $(p<0.01)$. 
A

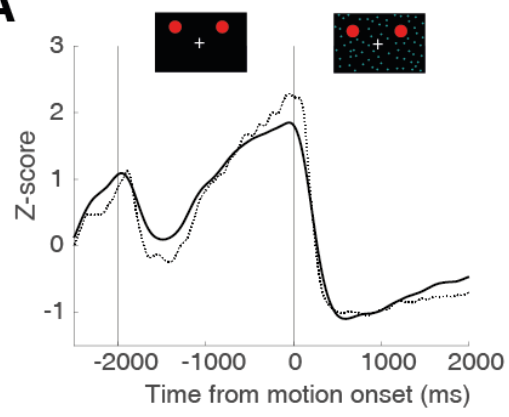

C

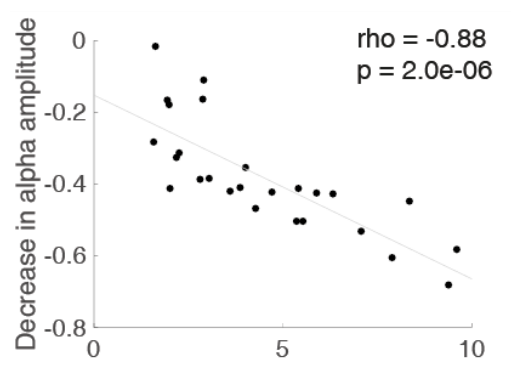

Individual prestimulus alpha amplitude

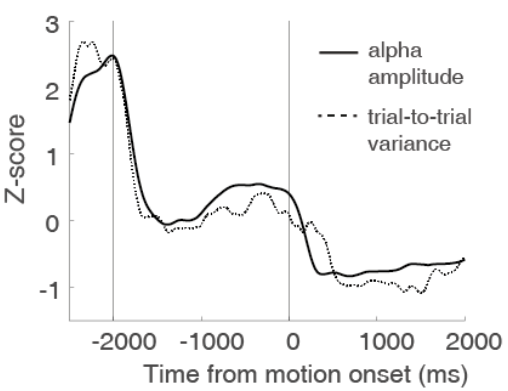

D

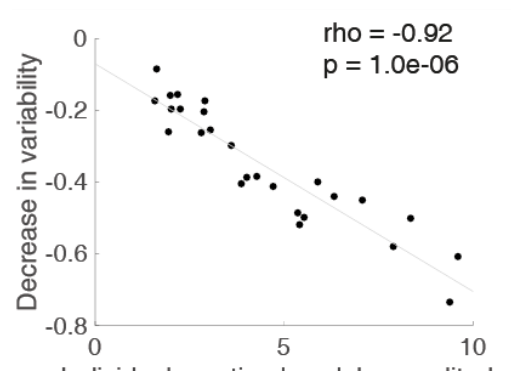

B

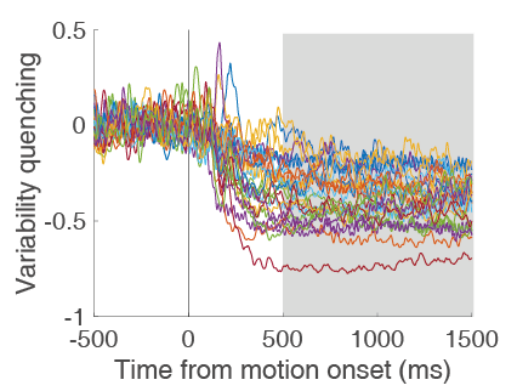

$\mathbf{E}$

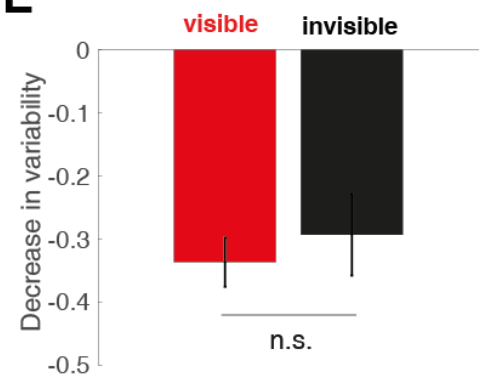

Figure 3. Quenching of trial-to-trial variability and relation to prestimulus alpha amplitude. A) Average variance across trials ( $z$-score, dotted line) and alpha amplitude (z-score, black line) over the course of experimental trials for two example subjects. B) Individual relative variance per subject (outlier corrected $>2 S D, N=26$ ) and post stimulus time window used to define the individual degree of variability quenching (black bar). C) Relation of decrease in alpha activity to individual alpha amplitude in the prestimulus interval, $\mathrm{N}=26$. Each dot represents one subject. Spearman's rank correlation coefficient and corresponding $p$ value. The gray line represents the least-squares fit. D) Relation of the individual degree of variability quenching to individual alpha amplitude in the prestimulus interval, $N=26$. Spearman's rank correlation coefficient and corresponding $p$ value. The gray line shows the leastsquares fit. E) Mean change in variance with RDM stimulus onset (trial-to-trail variability quenching) for visible and invisible trials across subjects $(\mathrm{N}=26)$. Error bars indicate $+/-1$ SEM.

Consistent with previous findings (Daniel et al., 2019), we found the individual magnitude of variability quenching to be significantly correlated with the decrease in alpha amplitude following the onset of the RDM stimulus (Spearman's rank correlation coefficient, rho $=0.87, p=0.000002, N=26$ ). Based on the similarities of previous findings associating both trial-to-trial variability quenching as well as prestimulus alpha amplitude with subsequent perceptual performance, we wondered whether the individual decrease in trial-to-trial variance as well as alpha amplitude was intrinsically related to the individual magnitude of ongoing alpha activity prior to stimulus onset. We found both the decrease in alpha amplitude (Spearman's rank correlation coefficient, rho $=-0.88, p=0.000002, N=26$ ) as well as the decrease in trial-to-trial variability (Spearman's rank correlation coefficient, rho $=-0.92, p=$ $0.000001, \mathrm{~N}=26$ ) to be significantly negatively correlated to the individual prestimulus alpha amplitude 
in the second preceding RDM stimulus onset (Figure 3C-D). Neither the magnitude of variance in the second preceding RDM onset nor during the stable period of RDM stimulus presentation differed significantly between invisible and visible trials (Wilcoxon signed-rank tests, prestimulus $p=0.70$, poststimulus $p=0.35$ ). Comparing the decrease in trial-to-trial variability between visible and invisible trials we observed no significant difference between perceptual states (Wilcoxon signed-rank tests, $p=$ $0.75, N=26$, Figure $3 E$ ), suggesting that variability quenching did not itself differentiate between perceptual outcomes.

\subsection{Discussion}

In the current study, we employed generalized flash suppression (GFS) (Wilke et al., 2003 p.20) to compare parieto-occipital prestimulus alpha activity of the human EEG between physically identical trials during which salient visual stimuli were either consciously perceived or suppressed from awareness. In contrast to previous studies that used visual masking and other perceptual threshold paradigms and have found prestimulus alpha power to be reduced for stimuli that are consciously perceived (Ergenoglu et al., 2004; Hanslmayr et al., 2007; Dijk et al., 2008; Benwell et al., 2017), we observed significantly lower alpha amplitudes prior to perceptual suppression compared to trials during which the target stimuli were reported as visible. One possible explanation for our result is that the varying perceptual outcomes of GFS despite identical physical stimulation conditions are due to spontaneous fluctuations in attentional state. Attentional modulations of neural activity serve to preferentially process behaviourally relevant stimuli while inhibiting the processing of competing distractors and previous research has found alpha activity to be reduced for attended compared to unattended stimuli (Sauseng et al., 2005; Kelly et al., 2006; Thut, 2006). Decreased alpha activity has been shown to reflect a state of increased neural excitability (Jensen and Mazaheri, 2010; lemi et al., 2017) and alpha activity has been associated with distractor suppression, showing anticipatory increases in alpha power corresponding to locations likely containing distractor information (Worden et al., 2000; Kelly et al., 2006; Wöstmann et al., 2019). In our case, reduced prestimulus alpha activity prior to RDM onset would thus predict enhanced processing of the surrounding motion stimulus in the same hemifield, rendering it more effective in suppressing the respective GFS target. Equivalently, when prestimulus alpha activity is high, the upcoming motion stimulus can be more successfully 
suppressed in order to maintain relevant (target) information and thus has little impact on the continued perception of the target. Unlike previous studies investigating the relationship between prestimulus alpha activity and visual awareness, we examined alpha activity prior to the onset of the motion stimulus rather than preceding the target itself, and it is thus conceivable that in paradigms in which a target stimulus does not reach awareness, such as backward masking, brief stimulus presentations and stimuli presented at low contrasts that can no longer be reliably distinguished, target visibility may depend on a certain degree of neural excitability prior to the onset of the target, whereas in the our case, increased neural excitability prior to the upcoming motion stimulus predicts its effectiveness to actively suppress an already consciously perceived target stimulus from awareness.

Given that in the current study we were not able to distinguish between modulations of alpha activity pertaining to the target and the suppressor stimulus presented in the same visual hemifields, another possible point of view is that the reduced alpha activity we observed prior to perceptual suppression via GFS reflects increased attentiveness towards the already present target stimulus, which subsequently facilitates its subjective disappearance. Behavioural evidence for this interpretation comes from previous studies using motion-induced blindness (MIB), a related perceptual suppression paradigm where salient static target stimuli spontaneously disappear during continuous viewing of the target and a moving surround (Bonneh et al., 2001). Specifically, using MIB in combination with an attentional cueing task requiring subjects to report hue changes in one of two target stimuli, Schölvinck and Rees demonstrated that directing spatial attention to a MIB target counterintuitively increases the probability of its disappearance compared to the unattended target (Scholvinck and Rees, 2009). Consistent observations were also made in a study entitled 'The more you attend the less you see': In a set of psychophysical experiments, the authors showed that both increasing attentional load of a central detection task as well as increasing the number of targets between which attentional resources were shared decreased the disappearance probability of individual targets (Carter et al., 2008). Similarly, target invisibility times have been reported to be significantly increased by a focused attention compared to a divided attention condition (Geng et al., 2007). Moreover it is notable that both GFS (Wilke et al., 2003) and MIB employ visually salient stimuli, and that increasing target contrast enhances MIB rather than hampering subjective disappearances (Bonneh et al., 2001). Previous psychophysical experiments identified several factors that influence the effectiveness of perceptual suppression: 
spatially specific target adaptation, spatial context including density of the RDM pattern, and the amount of local and global conflict between target and surround (Wilke et al., 2003). Specifically, several hundred milliseconds pre-adaptation without a change in target position or orientation, a small spatial distance between target and surround and a mismatch of the eye of target and surround are required to ensure immediate target suppression upon surround onset (Wilke et al., 2003, 2006). GFS shares this pre-adaptation requirement with other perceptual suppression illusions such as MIB and binocular rivalry flash suppression (BRFS), while being considerably shorter than Troxler fading or filling-in illusions (Kim and Blake, 2005; Bonneh et al., 2014). It is thus conceivable that the amplitude of alpha oscillations before motion onset could have influenced the probability of perceptual suppression by affecting target adaptation, which has previously been suggested to increase the relative salience of upcoming novel stimuli (McDermott et al., 2010). Theoretically different target adaptation levels reflected in alpha power differences before RDM onset could be due to a number of factors such as spontaneous neural fluctuations, microsaccades or visual attention. Interestingly, attention in turn enhances visual adaptation measured as perceptual afterimages: low-level as well as high-level figural and face aftereffects are stronger when attention is directed towards the adapter stimulus (Yeh et al., 1996; Lou, 2001; Rhodes et al., 2011).

In previous intracranial recordings in monkeys, Wilke and colleagues showed that perceptual suppression was reflected in alpha power of the local field potential (LFP) at target-responsive sites in striate and extrastriate visual cortex (Wilke et al., 2006; Maier et al., 2008). This robust modulation occurred in a time window shortly after RDM onset matching the estimated illusory disappearance time and shows reduced alpha power during perceptual suppression. Consistent modulations of intracranial spectral amplitude by perceptual suppression have also been observed in humans (Vidal et al., 2015). In the current study, we did not observe any visibility-related differences in alpha amplitude post RDM stimulus, but it is possible that such modulations are too local to be evident in parieto-occipital alpha activity that is largely driven by the full-field motion stimulus. In addition to methodological differences between intracranial electrophysiological recordings and those of EEG sum potentials that pool activity from neural populations representing both target and motion stimulus, there could be perceptual reportrelated factors that account for the absence of significant post RDM effects in our study (Storm et al., 2017). Specifically, in contrast to the previous GFS studies in monkeys (Wilke et al., 2006, 2009), in the present study subjects reported their percept only after passively viewing the RDM stimulus for 2 
seconds rather than indicating target disappearances immediately, and it is possible that the delayed report resulted in reduced task engagement during stimulus presentation. It is also noteworthy that we asked subjects to report the occurrence of subjective disappearances only after RDM presentation regardless of whether the target had reappeared, and thus cannot assume that the percept was stable during this time period.

In addition to lower alpha amplitudes prior to perceptual suppression, we observed a strong correlation between individual prestimulus alpha amplitude and the degree by which neural variability across trials declined with RDM onset. The magnitude of variability quenching has previously been shown to be a reproducible individual characteristic associated with individual perceptual abilities (Arazi et al., 2017b, 2017a) and recently, to reflect the amplitude of neural oscillations in the alpha/beta band that similarly decreases with visual stimulation (Daniel et al., 2019). Here, we provide evidence that these measures depend on the level of prestimulus alpha activity. Given that stimulus-induced decreases of trial-to-trial variability and alpha amplitude strongly correlate and both are related to the magnitude of alpha amplitudes prior to stimulus onset, it could be argued that the perceptual relevance of the relative rather than the absolute variance across trials (Arazi et al., 2017a) may be functionally rooted in prestimulus alpha oscillations. In our case, only prestimulus alpha amplitude significantly differentiated between perceptual outcomes while variability quenching did not, but more research is needed to understand the exact relationship between trial-to-trial variability and alpha amplitude and their consequences for perception.

While it seems evident that perceptual suppression critically depends on cortical state as indexed by prestimulus alpha oscillations in posterior cortex, several potential factors suppressing salient target stimuli from visual awareness remain unclear. Future studies specifically manipulating spatial attention could address the effect of attention on perceptual suppression and prestimulus alpha activity directly, as well as develop experimental designs that allow for the differentiation between modulations of alpha activity pertaining to the target and the motion stimulus. From the present study, we can conclude that reduced alpha activity predicts perceptual suppression, likely facilitating stronger processing of the upcoming motion stimulus and its effectiveness in suppressing the target. 


\subsection{References}

Arazi A, Censor N, Dinstein I (2017a) Neural Variability Quenching Predicts Individual Perceptual Abilities. The Journal of Neuroscience 37:97-109.

Arazi A, Gonen-Yaacovi G, Dinstein I (2017b) The Magnitude of Trial-By-Trial Neural Variability Is Reproducible over Time and across Tasks in Humans. eNeuro 4 Available at: https://www.ncbi.nlm.nih.gov/pmc/articles/PMC5739532/ [Accessed May 11, 2019].

Arieli A, Sterkin A, Grinvald A, Aertsen A (1996) Dynamics of ongoing activity: explanation of the large variability in evoked cortical responses. Science 273:1868-1871.

Benwell CSY, Tagliabue CF, Veniero D, Cecere R, Savazzi S, Thut G (2017) Prestimulus EEG Power Predicts Conscious Awareness But Not Objective Visual Performance. eNeuro 4 Available at: https://www.ncbi.nlm.nih.gov/pmc/articles/PMC5732016/ [Accessed April 29, 2019].

Bonneh YS, Cooperman A, Sagi D (2001) Motion-induced blindness in normal observers. Nature 411:798-801.

Bonneh YS, Donner TH, Cooperman A, Heeger DJ, Sagi D (2014) Motion-Induced Blindness and Troxler Fading: Common and Different Mechanisms Lu Z-L, ed. PLoS ONE 9:e92894.

Brainard DH (1997) The Psychophysics Toolbox. Spat Vis 10:433-436.

Carter O, Luedeman R, Mitroff S, Nakayama K (2008) Motion induced blindness: The more you attend the less you see. Journal of Vision 8:237-237.

Churchland MM et al. (2010) Stimulus onset quenches neural variability: a widespread cortical phenomenon. Nature Neuroscience 13:369-378.

Clark JH (1924) The Ishihara Test for Color Blindness. American Journal of Physiological Optics 5:269-276.

Cohen MR, Maunsell JHR (2009) Attention improves performance primarily by reducing interneuronal correlations. Nature Neuroscience 12:1594-1600.

Daniel E, Meindertsma T, Arazi A, Donner TH, Dinstein I (2019) The Relationship between Trial-byTrial Variability and Oscillations of Cortical Population Activity. Sci Rep 9:1-11.

Dijk H van, Schoffelen J-M, Oostenveld R, Jensen O (2008) Prestimulus Oscillatory Activity in the Alpha Band Predicts Visual Discrimination Ability. J Neurosci 28:1816-1823.

Dinstein I, Heeger DJ, Behrmann M (2015) Neural variability: friend or foe? Trends in Cognitive Sciences 19:322-328.

Ergenoglu T, Demiralp T, Bayraktaroglu Z, Ergen M, Beydagi H, Uresin Y (2004) Alpha rhythm of the EEG modulates visual detection performance in humans. Cognitive Brain Research 20:376-383.

Foxe JJ, Snyder AC (2011) The Role of Alpha-Band Brain Oscillations as a Sensory Suppression Mechanism during Selective Attention. Front Psychol 2 Available at: https://www.frontiersin.org/articles/10.3389/fpsyg.2011.00154/full [Accessed April 29, 2019].

Geng H, Song Q, Li Y, Xu S, Zhu Y (2007) Attentional modulation of motion-induced blindness. Chin Sci Bull 52:1063-1070.

HansImayr S, Aslan A, Staudigl T, Klimesch W, Herrmann CS, Bäuml K-H (2007) Prestimulus oscillations predict visual perception performance between and within subjects. Neurolmage $37: 1465-1473$.

lemi L, Chaumon M, Crouzet SM, Busch NA (2017) Spontaneous Neural Oscillations Bias Perception by Modulating Baseline Excitability. J Neurosci 37:807-819. 
Jensen O, Mazaheri A (2010) Shaping Functional Architecture by Oscillatory Alpha Activity: Gating by Inhibition. Frontiers in Human Neuroscience 4 Available at:

http://journal.frontiersin.org/article/10.3389/fnhum.2010.00186/abstract [Accessed July 25, 2019].

Keliris GA, Logothetis NK, Tolias AS (2010) The Role of the Primary Visual Cortex in Perceptual Suppression of Salient Visual Stimuli. J Neurosci 30:12353-12365.

Kelly SP, Lalor EC, Reilly RB, Foxe JJ (2006) Increases in Alpha Oscillatory Power Reflect an Active Retinotopic Mechanism for Distracter Suppression During Sustained Visuospatial Attention. Journal of Neurophysiology 95:3844-3851.

Kim CY, Blake R (2005) Psychophysical magic: rendering the visible "invisible." Trends Cogn Sci 9:381-388.

Kleiner M (n.d.) What's new in Psychtoolbox-3? :89.

Klimesch W, Sauseng P, Hanslmayr S (2007) EEG alpha oscillations: The inhibition-timing hypothesis. Brain Research Reviews 53:63-88.

Lange J, Oostenveld R, Fries P (2013) Reduced Occipital Alpha Power Indexes Enhanced Excitability Rather than Improved Visual Perception. Journal of Neuroscience 33:3212-3220.

Lou L (2001) Effects of voluntary attention on structured afterimages. Perception 30:1439-1448.

Macdonald JSP, Mathan S, Yeung N (2011) Trial-by-Trial Variations in Subjective Attentional State are Reflected in Ongoing Prestimulus EEG Alpha Oscillations. Front Psychol 2 Available at: https://www.frontiersin.org/articles/10.3389/fpsyg.2011.00082/full [Accessed April 29, 2019].

Maier A, Wilke M, Aura C, Zhu C, Ye FQ, Leopold DA (2008) Divergence of fMRI and neural signals in V1 during perceptual suppression in the awake monkey. Nature Neuroscience 11:1193-1200.

McDermott KC, Malkoc G, Mulligan JB, Webster MA (2010) Adaptation and visual salience. Journal of Vision 10:17-17.

Mitchell JF, Sundberg KA, Reynolds JH (2007) Differential attention-dependent response modulation across cell classes in macaque visual area V4. Neuron 55:131-141.

Mitchell JF, Sundberg KA, Reynolds JH (2009) Spatial Attention Decorrelates Intrinsic Activity Fluctuations in Macaque Area V4. Neuron 63:879-888.

Mo J, Schroeder CE, Ding M (2011) Attentional Modulation of Alpha Oscillations in Macaque Inferotemporal Cortex. J Neurosci 31:878-882.

Oostenveld R, Fries P, Maris E, Schoffelen J-M (2011) FieldTrip: Open Source Software for Advanced Analysis of MEG, EEG, and Invasive Electrophysiological Data. Computational Intelligence and Neuroscience 2011:1-9.

Poland E, Donner TH, Müller K-M, Leopold DA, Wilke M (2019) Thalamus exhibits less sensory variability quenching than cortex. Sci Rep 9:1-12.

Ress D, Backus BT, Heeger DJ (2000) Activity in primary visual cortex predicts performance in a visual detection task. Nature Neuroscience 3:940-945.

Rhodes G, Jeffery L, Evangelista E, Ewing L, Peters M, Taylor L (2011) Enhanced attention amplifies face adaptation. Vision Res 51:1811-1819.

Romei V, Gross J, Thut G (2010) On the Role of Prestimulus Alpha Rhythms over Occipito-Parietal Areas in Visual Input Regulation: Correlation or Causation? J Neurosci 30:8692-8697.

Romei V, Rihs T, Brodbeck V, Thut G (2008) Resting electroencephalogram alpha-power over posterior sites indexes baseline visual cortex excitability. Neuroreport 19:203-208. 
Samaha J, lemi L, Postle BR (2017) Prestimulus alpha-band power biases visual discrimination confidence, but not accuracy. Consciousness and Cognition 54:47-55.

Sauseng P, Klimesch W, Stadler W, Schabus M, Doppelmayr M, Hanslmayr S, Gruber WR, Birbaumer N (2005) A shift of visual spatial attention is selectively associated with human EEG alpha activity. Eur J Neurosci 22:2917-2926.

Scholvinck ML, Rees G (2009) Attentional influences on the dynamics of motion-induced blindness. Journal of Vision 9:38-38.

Schölvinck ML, Saleem AB, Benucci A, Harris KD, Carandini M (2015) Cortical State Determines Global Variability and Correlations in Visual Cortex. J Neurosci 35:170-178.

Schurger A, Sarigiannidis I, Naccache L, Sitt JD, Dehaene S (2015) Cortical activity is more stable when sensory stimuli are consciously perceived. Proceedings of the National Academy of Sciences 112:E2083-E2092.

Storm JF, Boly M, Casali AG, Massimini M, Olcese U, Pennartz CMA, Wilke M (2017) Consciousness Regained: Disentangling Mechanisms, Brain Systems, and Behavioral Responses. J Neurosci 37:10882-10893.

Thut G (2006) -Band Electroencephalographic Activity over Occipital Cortex Indexes Visuospatial Attention Bias and Predicts Visual Target Detection. Journal of Neuroscience 26:9494-9502.

Vidal JR, Perrone-Bertolotti M, Kahane P, Lachaux J-P (2015) Intracranial spectral amplitude dynamics of perceptual suppression in fronto-insular, occipito-temporal, and primary visual cortex. Front Psychol 5 Available at: https://www.ncbi.nlm.nih.gov/pmc/articles/PMC4295601/ [Accessed November 10, 2019].

Wilke M, Logothetis NK, Leopold DA (2003) Generalized Flash Suppression of Salient Visual Targets. Neuron 39:1043-1052.

Wilke M, Logothetis NK, Leopold DA (2006) Local field potential reflects perceptual suppression in monkey visual cortex. PNAS 103:17507-17512.

Wilke M, Mueller K-M, Leopold DA (2009) Neural activity in the visual thalamus reflects perceptual suppression. Proceedings of the National Academy of Sciences 106:9465-9470.

Worden MS, Foxe JJ, Wang N, Simpson GV (2000) Anticipatory biasing of visuospatial attention indexed by retinotopically specific alpha-band electroencephalography increases over occipital cortex. J Neurosci 20:RC63.

Wöstmann M, Alavash M, Obleser J (2019) Alpha Oscillations in the Human Brain Implement Distractor Suppression Independent of Target Selection. J Neurosci 39:9797-9805.

Yeh SL, Chen IP, De Valois KK, De Valois RL (1996) Figural aftereffects and spatial attention. J Exp Psychol Hum Percept Perform 22:446-460. 


\subsection{Supplementary}

\section{Supplementary Information S1: Cluster-permutation test}

In addition to the analysis of the average alpha amplitude during the second preceding RDM onset, we performed a spatio-temporal clustering analysis allowing us to identify statistically significant differences in prestimulus alpha amplitude between visible and invisible conditions independent of the a priori selected electrodes and 1 second time interval. To this end we divided the second prior to RDM onset in $100 \mathrm{~ms}$ windows. A cluster-level statistic was calculated based on these time windows of interest and all 63 sensors, requiring a minimum number of two neighbouring channels and consecutive time points. In order to obtain cluster-corrected $\mathrm{p}$ values we employed a Monte Carlo permutation with 500 iterations. The cluster-permutation test revealed a significant difference between visible and invisible conditions (cluster-level statistic $=42.35, \mathrm{p}=0.04, \mathrm{~N}=27$ ) that was most prominent between $600-400$ ms prior to RDM onset in in right occipital and frontal electrodes.
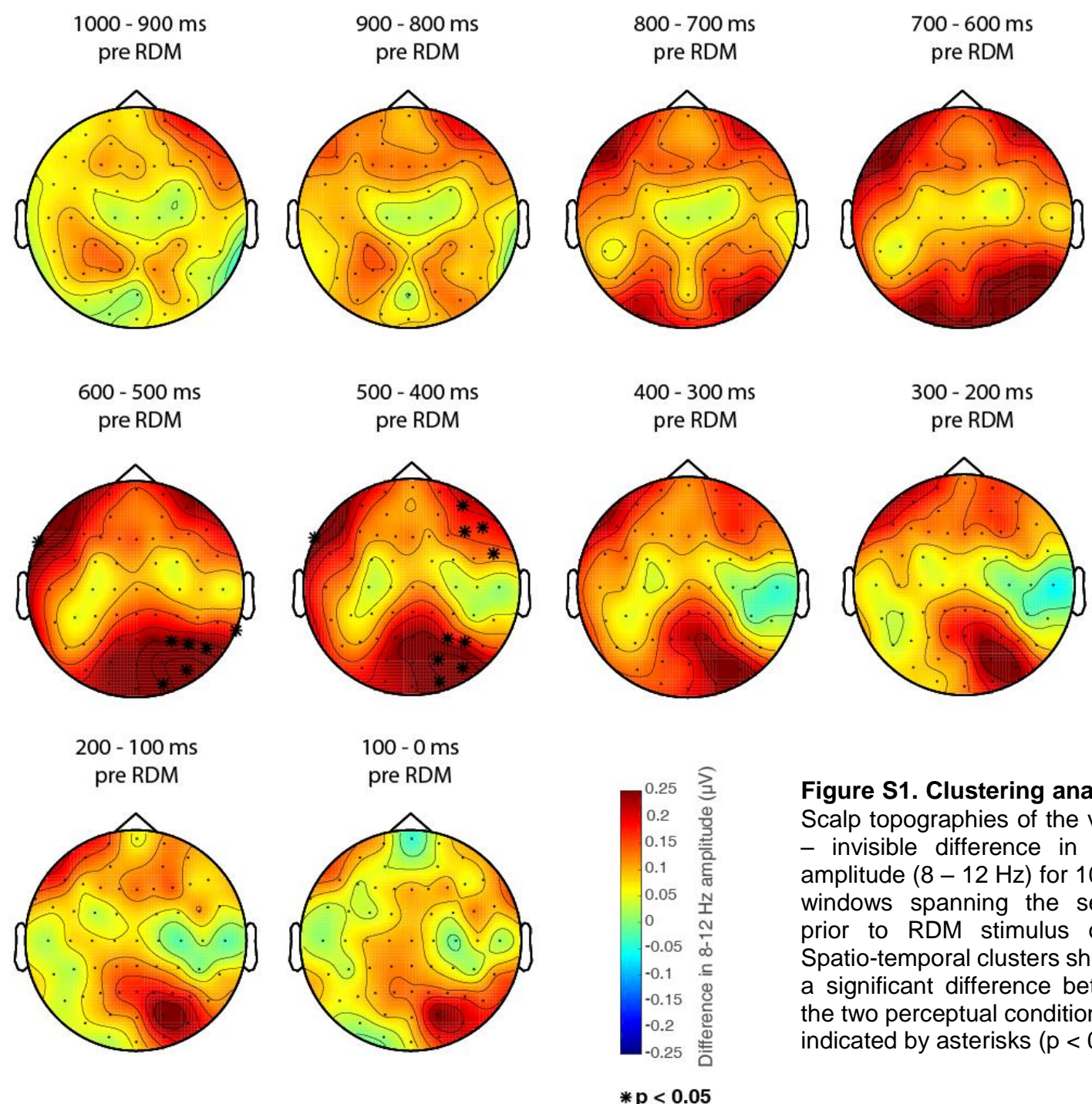

Figure S1. Clustering analysis. Scalp topographies of the visible - invisible difference in alpha amplitude $(8-12 \mathrm{~Hz})$ for $100 \mathrm{~ms}$ windows spanning the second prior to RDM stimulus onset. Spatio-temporal clusters showing a significant difference between the two perceptual conditions are indicated by asterisks $(p<0.05)$. 


\section{Supplementary Information S2: Unilateral target disappearances}

Table 1 summarizes mean alpha amplitudes in the second prior to RDM stimulus onset for trials in which only either the left or the right target stimulus disappeared for left and right hemisphere ROls. Since previous studies investigating the lateralization of alpha activity have focused on more parietal sensors (Thut et al., 2006) we additionally compared unilateral target disappearances between left and right hemispheric ROls that included more parietal electrodes (Table 2), but also did not observe a significant difference between left and right unilateral conditions when considering parietal sensors.

\begin{tabular}{|c|c|c|}
\hline & $\begin{array}{c}\text { Left hemisphere } \\
\text { (01 / PO3 / PO7) }\end{array}$ & $\begin{array}{c}\text { Right hemisphere } \\
\text { (O2 / PO4 / PO8) }\end{array}$ \\
\hline $\begin{array}{c}\text { Unilateral left } \\
\text { disappearances }\end{array}$ & $4.32+$ - SD 2.69 & $4.47+$ - SD 2.69 \\
\hline $\begin{array}{c}\text { Unilateral right } \\
\text { disappearances }\end{array}$ & $4.15+/-$ SD 2.29 & $4.40+/-$ SD 2.43 \\
\hline p value & 0.63 & 0.79 \\
\hline
\end{tabular}

Table 1. Mean alpha $(8-12 \mathrm{~Hz})$ amplitudes in the second preceding RDM onset and standard deviations in the left and right hemisphere for unilateral target disappearances. Significance between unilateral left and unilateral right target disappearances as determined by Wilcoxon signed-rank tests, $\mathrm{N}=21$.

\begin{tabular}{|c|c|c|}
\hline & $\begin{array}{c}\text { Left hemisphere } \\
\text { (PO3 / P5 / P7) }\end{array}$ & $\begin{array}{c}\text { Right hemisphere } \\
\text { (PO4 / P6 / P8) }\end{array}$ \\
\hline $\begin{array}{c}\text { Unilateral left } \\
\text { disappearances }\end{array}$ & $3.89+/$ SD 2.12 & $4.16+/-$ SD 2.32 \\
\hline $\begin{array}{c}\text { Unilateral right } \\
\text { disappearances }\end{array}$ & $3.76+$ - SD 1.93 & $4.12+/-$ SD 2.20 \\
\hline p value & 0.50 & 0.71 \\
\hline
\end{tabular}

Table 2. Mean alpha $(8-12 \mathrm{~Hz})$ amplitudes in the second preceding RDM onset and standard deviations for unilateral target disappearances in more parietal left and right hemispheric electrodes. Significance between unilateral left and unilateral right target disappearances as determined by Wilcoxon signedrank tests, $\mathrm{N}=21$. 


\section{Supplementary Information S3: Frequency contributions to variability quenching}

Finally, we sought to identify the contributions of different physiological frequency bands to the overall decrease in variability. To this end we first bandpass filtered the data between $4-90 \mathrm{~Hz}$ as a reference. For better comparability with the $4-90 \mathrm{~Hz}$ broadband signal (black, Figure 2A), we chose an indirect approach and removed the influence of the different physiological frequency bands one at a time by band-stop in the theta $(4-7 \mathrm{~Hz})$, alpha $(8-12 \mathrm{~Hz})$, beta $(13-30 \mathrm{~Hz})$, low gamma $(31-50 \mathrm{~Hz})$ and high gamma $(51-90 \mathrm{~Hz})$ bands using a $4^{\text {th }}$ order Butterworth filter. We then computed the variance across trials of all perceptual conditions for the filtered signals (Figure S2A) as well as the relative variance as percent change from baseline to post RDM stimulus interval. Oscillations in the alpha frequency (red) constituted the largest contribution to prestimulus variance and also had the largest effect on variability quenching. Significant changes in magnitude of quenching following band-stop filtering occurred for the removal of theta $(44 \%$ of the full $4-90 \mathrm{~Hz}$ signal to $47 \%, \mathrm{p}=4.1000 \mathrm{e}-05)$, alpha $(44 \%$ to $24 \%, \mathrm{p}=$ 9.3386e-06) and low gamma (44\% to $50 \%, p=0.0059)$ frequencies, but not for beta ( $44 \%$ to $44 \%, p$ $=0.39$ ) and higher gamma $(44 \%$ to $49 \%, p=0.02$, Bonferroni corrected $p=0.01)$. Figure S2B shows the average prestimulus amplitudes for the considered frequency bands in the second preceding RDM onset, scaling to the respective contributions to prestimulus variance.
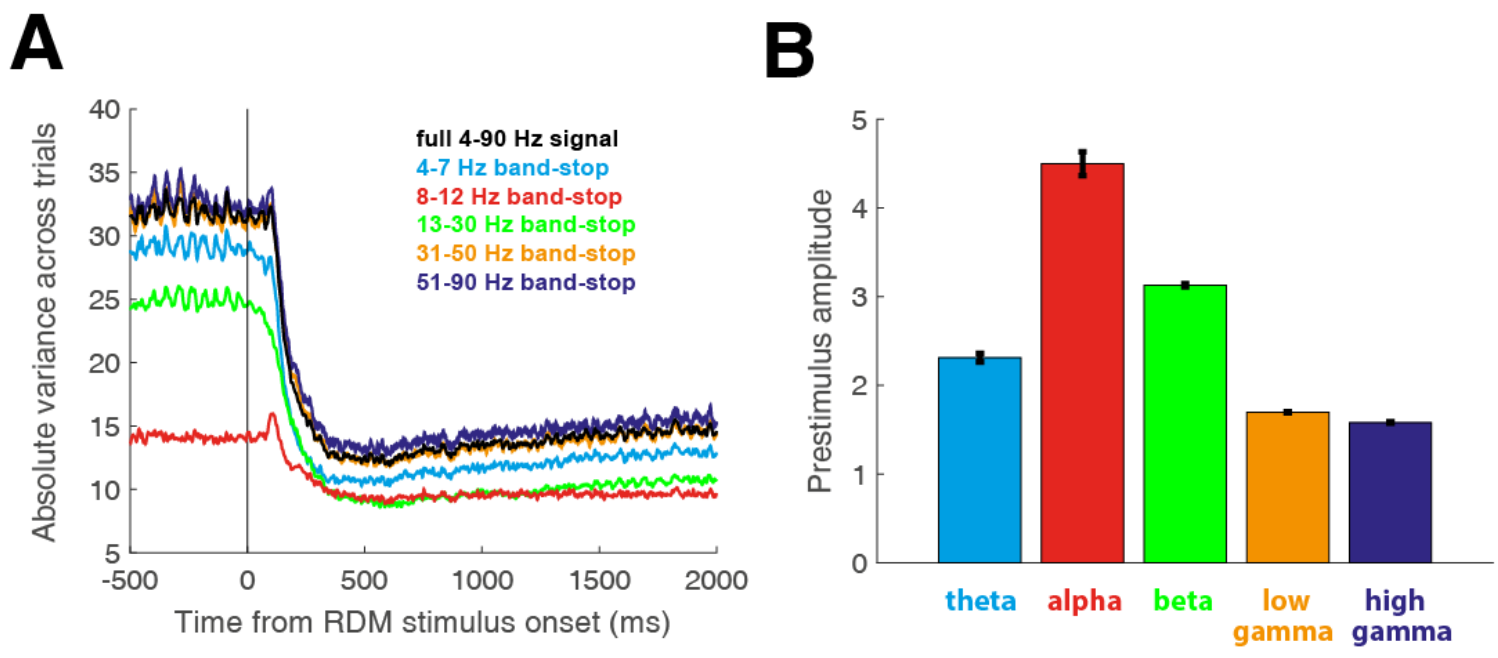

Figure S2. Contributions of different frequency bands to variability quenching in visual cortex. A) Average absolute variance around RDM stimulus onset for band-stop filtered broad-band signal from which the influence of different physiological frequency bands has been removed $(\mathrm{N}=27)$. B) Average prestimulus amplitude of oscillations in the corresponding physiological frequency bands theta $(4-7 \mathrm{~Hz}$, blue), alpha (8-12 Hz, red), beta (13$30 \mathrm{~Hz}$, green) as well as low (31-50 Hz, orange) and high gamma (51-90 Hz, purple). Error bars indicate +/- 1 SEM. 


\section{Chapter V}

\section{Attentional influences on perceptual suppression}

Eva Poland ${ }^{1}$ and Melanie Wilke $1,2,3$

${ }^{1}$ Department of Cognitive Neurology, University of Goettingen, Robert-Koch-Str. 40, Goettingen, 37075, Germany

${ }^{2}$ German Primate Center, Leibniz Institute for Primate Research, Kellnerweg 4, Goettingen, 37077, Germany

${ }^{3}$ Leibnitz Science Campus Primate Cognition, Kellnerweg 4, Goettingen, 37077, Germany

\section{Author contributions}

E. Poland developed the study concept and task and collected the data. E. Poland analyzed the data. M. Wilke interpreted the data and conceptually contributed to the analysis process. E. Poland drafted the manuscript: 


\subsection{Abstract}

Whether visual awareness and visual attention can be thought of as largely synonymous or should instead be treated as distinct neurological phenomena is still a subject of considerable debate. Evidence suggesting that both can at least to some degree occur independent of each other supports the concept of separate underlying mechanisms, but distinguishing neural correlates of awareness from other confounding factors such as attention is still a considerable challenge for research. Here, we combined the generalized flash suppression (GFS) paradigm with a spatial attention task in order to comparatively investigate the role of posterior alpha band activity in awareness and attention. We show that attention directed to one of two GFS targets presented in the left and right visual hemifield increases its probability to be subsequently perceptually suppressed. In accordance with previous literature the spatial attention task reduced alpha activity in the hemisphere contralateral to the attended target, which in turn predicted subsequent target disappearances. Attentional and perceptual modulations of alpha activity were not distinguishable by their affected frequency ranges and a full factorial analysis did not show a significant interaction between attention and awareness related effects on alpha amplitude. Our results suggest that the phenomenological outcome of perceptual suppression paradigms is dependent the attentional state prior to the onset of the suppressing motion stimulus, and that predictive alpha activity modulations associated with perceptual suppression reflect attentional prerequisites for visual awareness rather than awareness itself.

\subsection{Introduction}

Intuitively, awareness is often assumed to be synonymous with the focus of attention, that is what we selectively attend to at any given moment will determine our subjective conscious experience at this moment. Since phenomenal awareness has been studied more systematically by cognitive sciences, this view has been challenged by a number of researchers arguing that visual awareness and visual attention should instead be thought of as separate phenomena (Lamme, 2003). One of the main arguments for a distinction between the two concepts is that attentional modulations of neural signals can occur independent of awareness, while conscious perception can occur in the near absence of attention (Koch and Tsuchiya, 2007). Neurologically, the case has been made that attention enhances 
the processing of sensory signals in a feedforward fashion along the pathways of the sensory system, while phenomenal awareness requires additional recurrent processing within sensory processing stages as well as between sensory cortices and other regions of the brain (Lamme, 2003). Neural mechanisms underlying visual awareness are typically studied using dynamic illusions such as motion induced blindness (MIB) or variants of flash suppression by which salient stimuli are perceptually suppressed from awareness while physical stimulus input is kept constant (Kim and Blake, 2005). The employment of such illusions thus allows the experimenter to compare brain states during which the same stimulus is either perceived or subjectively invisible. Psychophysical studies using MIB have found that attention directed towards a target stimulus counterintuitively increases its probability to be perceptually suppressed rather than remain visible (Geng et al., 2007; Carter et al., 2008; Schölvinck and Rees, 2009), arguing that attentional state is critical for the dynamics of perceptual suppression. In a previous study (Chapter IV, unpublished) we could show that during generalized flash suppression (GFS), a similar visual illusion during which the perceptual suppression of initially presented static targets is induced by the onset of a surrounding motion stimulus, subjective target disappearances were predicted by reduced parieto-occipital alpha activity. The amplitude of parieto-occipital alpha rhythms is known to be lower over the hemisphere contralateral to the attended stimulus location when spatial attention is covertly directed to stimuli presented in the left or right visual hemifield (Worden et al., 2000; Sauseng et al., 2005; Kelly et al., 2006; Thut, 2006) and alpha activity reliably correlates with fluctuations in overall attentiveness inferred by subject reports (Macdonald et al., 2011). In addition to their function in selective anticipatory attention, reduced alpha amplitudes have further been associated with increased cortical excitability (Lange et al., 2013; lemi et al., 2017) and predict the conscious perception of visual stimuli that are presented at the perceptual threshold or under masking conditions (Ergenoglu et al., 2004; Hanslmayr et al., 2007; Dijk et al., 2008; Benwell et al., 2017), suggesting that the level of alpha activity over posterior cortex prior to stimulus onset is a determining factor for subsequent perceptual performance. In the present study we combined the bilateral GFS paradigm with a spatial attention task in order to investigate the effect of attention on perceptual suppression and to comparatively examine the role of posterior alpha band activity in mediating awareness and attention. Specifically, we hypothesized that increased attention towards either one of two target stimuli presented in the left and right hemifield would increase the disappearance probability of the attended target relative to the unattended target, as well as reduce parieto-occipital alpha amplitudes in the contralateral 
hemisphere. We aimed to design the experiment such that alpha amplitudes were modulated prior to the onset of the random dot motion (RDM) stimulus that induced perceptual suppression, allowing us to test the effect of alpha amplitude on subjective disappearance probabilities.

\subsection{Methods}

\section{Subjects}

A total of 30 healthy right handed subjects participated in the current study (16 male / 14 female, 18 to 50 years of age). All subjects gave their informed consent to and were rewarded for their participation in the study. For the analysis of EEG data we required a minimum number of 15 trials in each perceptual condition of the full factorial design, resulting in a cohort of 17 subjects (6 male / 11 female).

\section{Experimental procedure}

EEG data were recorded in a single session. Prior to the experiment subjects performed 20 practice trials in order to familiarize themselves with the task. We carefully instructed subjects to indicate target disappearances based on whether a target stimulus had disappeared completely and only the black background was perceived, regardless of whether perceptual suppression persisted until the end of the trial or the target reappeared within the 1 second of RDM stimulus presentation. Following EEG preparation, subjects completed 6 experimental blocks, each lasting approximately 12 minutes. Subjects were seated in front of a $60 \times 34 \mathrm{~cm}$ computer screen and placed their head on a chin rest with an eye to screen distance of $70 \mathrm{~cm}$. During the experiment, lights in the recording room were turned off and black curtains were used to screen the subject from extraneous light. Between blocks subjects had the opportunity to take brief breaks. All experimental procedures were approved by the ethics committee of the University Medicine Göttingen (UMG, Germany).

\section{Stimuli and task}

In order to investigate attentional effects on perceptual suppression we combined the generalized flash suppression (GFS) paradigm with a spatial attention task (Figure 1). Stimuli were programmed and presented in Matlab 2015b (The MathWorks Inc., Natick MA, USA) using Psychtoolbox-3 (Brainard, 1997). Each trial began with central fixation for 1 second, followed by a visual cue consisting of a small 
arrowhead attached to the fixation cross which pointed either to the left or to the right, indicating the to be attended visual hemifield. Subjects were then presented with two salient target stimuli consisting of red Gabor gratings (size $3^{\circ}$ of visual angle) in the left and right visual hemifield ( $7^{\circ}$ of visual angle horizontal distance from center, $3^{\circ}$ of visual angle vertical distance from center). The targets were only presented to one eye by means of red-green anaglyphic glasses (red filter left eye, green filter right eye). During this stimulus onset asynchrony (SOA) interval prior to motion onset, subjects reported orientation changes in the previously cued target with a mouse click as soon as the change occurred. Orientation changes consisted of either clockwise or counter-clockwise rotations of $20^{\circ}$ and the number of orientation changes on a given trial ranged from 0 to 2 with the same number of changes occurring in the left and right target at different times during the SOA interval. After 2 seconds, a random dot motion (RDM) pattern consisting of 2000 green dots moving at a speed of $10 \%$ second was shown to the respective other eye for 2 seconds, which resulted in the subjective disappearance of one or both target stimuli in a subset of trials. Following the end of stimulus presentation, subjects were prompted to report their perception, that is the subjective disappearance of both, neither, the right or the left target stimulus, with a mouse click. In addition to the experimental trials (600 trials), each subject performed 240 control trials intermixed with the experimental trials in which either the left or the right target stimulus was physically removed following RDM onset.

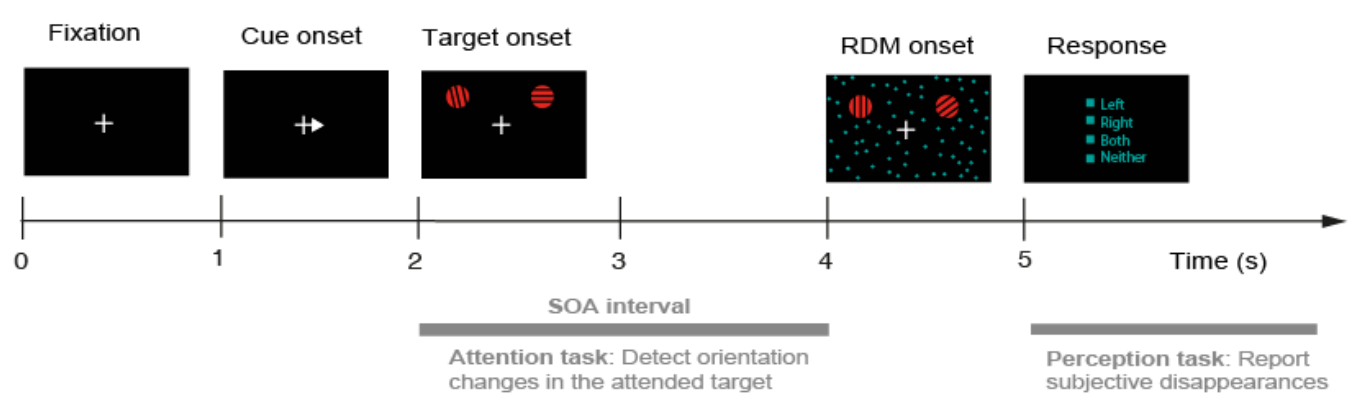

Figure 1. Experimental paradigm combining generalized flash suppression (GFS) with a spatial attention task. Each experimental trail starts with central fixation for one second, followed by a visual cue indicating the attended side with a small arrow. A second later two target stimuli consisting of red Gabor gratings are presented in the left and right visual hemifield. During the experiment red and green stimuli are viewed monocularly via red-green anaglyphic glasses. The targets are presented for a stimulus onset asynchrony (SOA) interval of 2 seconds prior to RDM stimulus onset. During the SOA interval subjects perform a spatial attention task whereby orientation changes in the previously cued target are reported immediately by a mouse click. After 2 seconds, the green RDM stimulus is presented to the other eye, thereby probabilistically inducing subjective invisibility of one or both targets on a subset of trials. After 1 second of RDM presentation, subjects are prompted to report whether the left, the right, neither or both targets disappeared. 


\section{Behavioral data analysis}

Subjects' performance on the change detection task was calculated as the probability of correct detections (hits) without considering false alarms, which we estimated separately as the probability of responses in the absence of an orientation change. The overall probability of perceptual suppression was calculated as the percentage of experimental trials on which any subjective disappearance, either unilateral (disappearances of the left or the right target) or bilateral (disappearance of both targets), occurred. To assess the influence of the physical orientation changes on perceptual suppression we calculated unilateral as well as bilateral disappearance probabilities as a function of the number of target orientation changes on a given trial. We tested the effect of spatial attention on perceptual suppression by comparing the probabilities of unilateral disappearances between attention conditions using Wilcoxon signed-rank tests for the left and the right target stimulus separately. To ensure the spatial attention task had not merely affected the probability of subjects reporting target disappearances, we examined control trials in which either the left or the right target had been physically removed from screen following RDM onset and similarly compared the percentages of correctly reported physical removals of the left or the right target between attention conditions.

\section{EEG acquisition and preprocessing}

EEG activity was recorded with a 64-electrode cap (63 EEG channels and 1 ECG channel, 64-Channel Standard BrainCap, Brain Products, Gilching, Germany) using BrainVision Recorder (Brain Products, Gilching, Germany). Electrode impedances were kept below $20 \mathrm{k} \Omega$ throughout the experiment. EEG data were preprocessed and analyzed using the Fieldtrip toolbox (Oostenveld et al., 2011) and customwritten software in Matlab 2015b (The MathWorks Inc., Natick MA, USA). The data were recorded at a sampling rate of $1000 \mathrm{~Hz}$ and bandpass filtered between $0.1-250 \mathrm{~Hz}$. Trials containing muscle artefacts, jumps or clipping artefacts were identified automatically and rejected following visual inspection. An independent component analysis (ICA) was performed to identify eye movement artefacts and eye movement related components were removed. The data were then re-referenced to a common reference. 


\section{EEG data analysis}

Our aim in designing the present experiment was to compare modulations of parieto-occipital alpha amplitudes induced by the spatial attention task and modulations of alpha amplitude that differentiated between subsequent perceptual outcomes of GFS. Previous studies investigating the effects of spatial attention on alpha activity have shown that directing attention to one side of space is associated with reduced alpha amplitudes in the contralateral hemisphere processing the attended hemifield relative to the ipsilateral hemisphere processing the to be ignored hemifield (see for example Kelly et al., 2006, Thut et al., 2006). We thus presented target stimuli in the left and right visual hemifield and focused our analyses on regions of interest (ROIs) in the right and left hemisphere respectively, which allowed us to sort the available trials with respect only to the contralateral target stimulus. We selected the parietooccipital electrodes PO7, P5 and P7 for the left and the electrodes PO8, P6 and P8 for the right hemispheric ROI based on an earlier publication that examined attentional modulations of alpha amplitude using a similar electrode cap layout as in our study (Thut, 2006). To confirm that our initial electrode selection had targeted brain regions that were modulated by the spatial attention task, we calculated 8-12 Hz alpha band amplitudes for each of the 63 electrodes and attention conditions separately and inspected scalp topographies representing the difference in alpha amplitude between attention conditions (left attended - right attended) for the second immediately following cue onset (post cue window) as well as for the second immediately preceding RDM stimulus onset (pre RDM window) on which we focused our analyses. For the calculation of alpha amplitudes, the data of the left and right hemisphere ROI electrodes were bandpass filtered at $8-12 \mathrm{~Hz}$ with a $4^{\text {th }}$ order Butterworth filter and subsequently Hilbert transformed. For statistical analysis and time courses of alpha amplitude we then considered the absolute values of the Hilbert transform equivalent to the envelope of the filtered signal and averaged over the selected electrodes.

To test for main effects of spatial attention we compared average alpha amplitudes within a given ROI in the post cue window as well as in the pre RDM window between attention conditions regardless of perceptual outcome. To determine whether there was a significant difference in alpha amplitude in the pre RDM window predicting the perceptual outcomes of GFS, we compared experimental trials in which the target stimulus contralateral to the considered ROI had been successfully perceptually suppressed or remained subjectively visible within ROls regardless of attentional condition. Statistical comparisons were carried out using Wilcoxon signed-rank tests. We then further sorted all available experimental 
trials into the conditions of the full factorial design for the left hemisphere ROI / right target stimulus and for the right hemisphere ROI / left target stimulus separately based on whether the respective contralateral target had been visible or invisible and attended or unattended on a given trial. For the left hemisphere ROI and the right target mean trial numbers for the factorial conditions were 148 +/- SD 55 visible-attended, 105 +/- SD 44 invisible-attended, 168 +/- SD 60 visible-unattended, 80 +/- SD 51 invisible-unattended. For the right hemisphere ROI and the left target mean trial numbers were $172+/-$ SD 50 visible-attended, 75 +/- SD 41 invisible-attended, 196 +/- SD 49 visible-unattended, 56 +/- SD 36 invisible-unattended.

In order to test for a possible interaction between attentional and perceptual modulations of alpha amplitude we performed a two-way repeated measures ANOVA with the factors attention and awareness, each varying on two levels (visible/invisible and attended/unattended), on the average alpha amplitudes across the pre RDM window for the left and for the right hemisphere ROI separately. Finally, we wondered whether the modulation of alpha amplitude by spatial attention and the modulation predicting subsequent perceptual suppression which we had tested on the average of the frequency range of 8 to $12 \mathrm{~Hz}$ differed in the affected frequencies. We thus calculated Fast Fourier Transforms (FFT) across the 1 second pre RDM window in a frequency range of 2 to $20 \mathrm{~Hz}$ using a Hanning window for each hemisphere ROI separately. Subjects' individual alpha frequencies (IAF) were determined as the individual peak frequency of the FFT of the pre RDM interval across all experimental trials across all parieto-occipital electrodes in a range between 5 and $15 \mathrm{~Hz}$. We then calculated FFTs for the left and right hemisphere ROIs for the attention conditions (contralateral target attended or unattended regardless of perceptual outcome) as well as for the perceptual conditions (contralateral target visible or invisible regardless of attention condition) centered around the IAF in the range IAF - $6 \mathrm{~Hz}$ to IAF + $6 \mathrm{~Hz}$. Based on the FFT we estimated attentional and perceptual modulation of low frequency amplitudes as the difference between conditions (visible - invisible and unattended - attended). For each subject we then determined the peak frequencies of attentional and perceptual modulation and tested for a difference using a Wilcoxon signed-rank test. 


\subsection{Results}

\section{Influence of spatial attention on perceptual suppression}

In the present study we sought to test the hypothesis that perceptual suppression is affected by attentional state. To this end we combined the generalized flash suppression (GFS) paradigm with a spatial attention task (Figure 1). Each trial began with central fixation, followed by a cue indicating the to be attended visual hemifield. A second later two target stimuli consisting of red Gabor gratings were presented in the left and right visual hemifield respectively. GFS relies on a stimulus onset asynchrony (SOA) of at least several hundred milliseconds between the initially presented target and the subsequent random dot motion (RDM) stimulus which then induces the perceptual suppression of the target (Wilke et al., 2003). Here we used an SOA of 2 seconds in order to manipulate subjects' attentional state prior to perceptual suppression. During the SOA interval subjects indicated small changes in the orientation of the cued target as soon as a rotation occurred. The onset of the surrounding RDM stimulus then induced perceptual suppression on a subset of trials, rendering the target stimuli subjectively invisible. After the motion stimulus had been presented for one second, participants were prompted to report whether any target disappearances had occurred.

The probability of perceptual suppression varied strongly between subjects and did not correlate with individual performance on the change detection task (Pearson's' correlation coefficient, $r=0.07, p=$ $0.70, \mathrm{~N}=30$, Figure $2 \mathrm{~A}$ ). The average detection rate of orientation changes across participants was $0.65+$ - SD 0.09 , while false alarms occurred at an average rate of $0.06+/$ - SD 0.06 , suggesting that subjects were actively engaged in the spatial attention task. Disappearances of both targets (bilateral disappearances) or of the left or the right target stimulus alone (unilateral disappearances) did not systematically vary with the number of orientation changes on a given trial (Figure 2B), indicating that the physical stimulus changes were a negligible influence on perceptual suppression in our case. In designing the experiment we had equated the number of orientation changes on a given trial between left and right hemifield targets, although the changes occurred at different times during the 2 second SOA interval in order to allow for the identification of responses specific to the cued target. Under these conditions unilateral disappearances of the left hemifield target were overall slightly likelier than unilateral right target disappearances but not significantly so (Wilcoxon signed-rank test, $\mathrm{p}=0.064, \mathrm{~N}$ $=30$, left target $0.14+/-$ SD 0.16 , right target $0.08+/-0.12$ ) 
We then sought to determine whether the attentional manipulation imposed by the change detection task had affected subsequent target disappearance probabilities in the left and right visual hemifield. Examining unilateral target disappearances we found the disappearance probability of a given target stimulus to be significantly higher when the target was attended compared to when it was unattended, which was the case for the left (Wilcoxon signed-rank test, $\mathrm{p}=0.001, \mathrm{~N}=30$, mean attended $0.13+/$ SD 0.12 , mean unattended $0.10+/-$ SD 0.11 , Figure $2 \mathrm{C}$ ) as well as for the right target stimulus (Wilcoxon signed-rank test, $\mathrm{p}=0.0002, \mathrm{~N}=30$, mean attended $0.09+/-\mathrm{SD} 0.09$, mean unattended $0.07+/$ SD 0.07 , Figure 2D). Did our attentional manipulation influence the probability of perceptual suppression itself or did it merely increase the probability of subjects reporting subjective target disappearances? In addition to and intermixed with experimental trials, participants also completed 240 control trials on which either the left or the right target stimulus was physically removed from screen following the onset of the RDM stimulus.

In order to determine whether the attentional manipulation had affected the likelihood of subjects reporting target disappearances, we compared subjects reporting target disappearances, we compared disappearance probabilities reported for these physical removals between attention conditions. Subjects reliably indicated physical removals as target disappearances throughout the experiment (Figure 2E-F) and removal detection rates did not statistically differ between the imposed attentional conditions for the left target (Wilcoxon signed-rank test, $p=0.19, \mathrm{~N}=30$, mean attended $0.97+/-0.05$ SD, mean unattended $0.97+/$ - SD 0.05, Figure 2E) or for the right target (Wilcoxon signed-rank test, $p$ $=0.72, \mathrm{~N}=30$, mean attended $0.96+/-\mathrm{SD} 0.06$, mean unattended $0.96+/-\mathrm{SD} 0.06$, Figure 2F), suggesting that spatial attention directed to the target stimulus had in fact increased the probability of perceptual suppression of the attended target rather than the frequency of subject reports. 

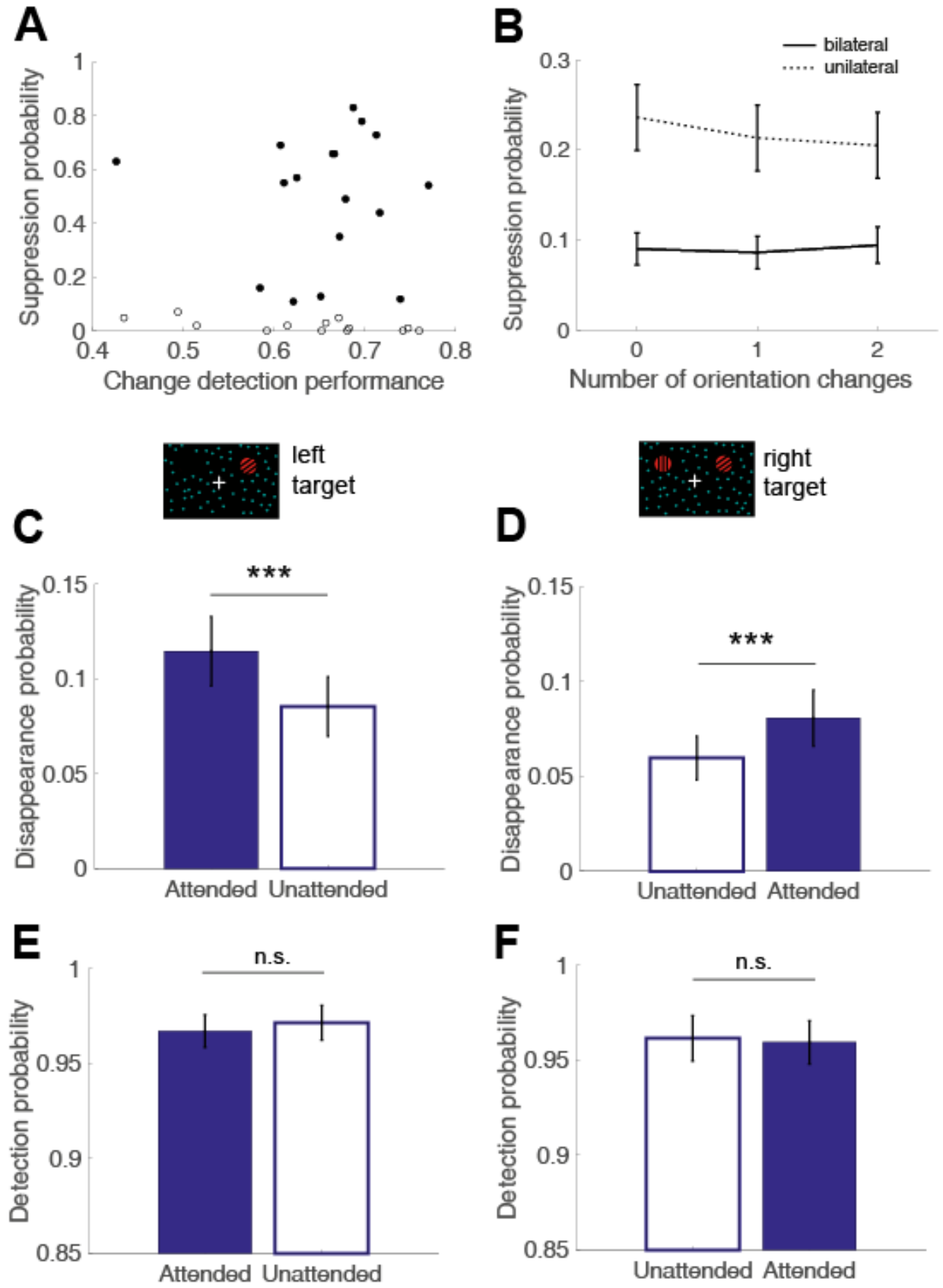

Figure 2. Effect of spatial attention on perceptual suppression. (A) Overall probability of bilateral or unilateral perceptual suppression for individual subjects as a function of individual performance on the attention task (probability of correct change detections, $N=30$ ). Subjects included in subsequent EEG analysis due to a sufficient trial numbers are marked as filled circles. (B) Probability of bilateral (dotted line) and unilateral (solid line) perceptual suppression across subjects $(\mathrm{N}=30)$. Error bars indicate +/- 1 SEM. (C) Probability of perceptual suppression of the left target alone across subjects ( $\mathrm{N}$ $=30$ ) for trials in which the left target was attended (filled bar) versus unattended (empty bar). ${ }^{* * *}$ indicates $p<=0.001$. (D) Disappearance probability of the right target alone across subjects $(N=30)$ for trials in which the right target was attended (filled bar) or unattended (empty bar). ${ }^{* * *}$ indicates $p<=$ 0.001. (E) Probability of correctly detected physical removals of the left target for trials in which the left target was attended (filled bar) versus unattended (empty bar). N.s. indicates no significant difference between attention conditions. (E) Probability of correctly detected physical removals of the right target for trials in which the right target was attended (filled bar) versus unattended (empty bar). N.s. indicates no significant difference between attention conditions. 
Modulation of alpha amplitude by spatial attention

We hypothesized that the effect of attentional manipulation on the disappearance probabilities of the GFS targets was due to spatial attention reducing alpha amplitudes prior to the onset of the RDM stimulus, which in turn predicted subsequent perceptual suppression of the attended target. We thus examined alpha $(8-12 \mathrm{~Hz})$ activity in left and right hemisphere regions of interest (ROIs) with regard to the target stimulus in the respective contralateral visual hemifield. Due to the fact that trial numbers were dependent on perceptual report we considered 17 subjects (marked with filled circles in Figure 2A) who had a minimum number of 15 trials in each perceptual condition for the analysis of EEG alpha amplitudes. Equivalent to the behavioral results obtained based on all 30 study participants, attended targets were significantly likelier to be perceptually suppressed in this cohort (Wilcoxon signed-rank tests, left target: $p=0.003, N=17$; right target: $p=0.001, N=17$ ).

We selected electrodes for the left (PO7, P5, P7) and right hemispheric ROls (PO8, P6, P8) based on previous literature examining attentional modulations of parieto-occipital alpha amplitude (Thut et al., 2006). The topographies of the difference in alpha amplitude between spatial attention conditions (left attended - right attended) for the second following cue onset (Figure $\mathbf{3 A}$ ) as well as for the second prior to RDM onset (Figure 3B) indicate that the attentional modulations of alpha amplitude were largest around the chosen ROIs.

Figure 3C-D shows the time courses of alpha amplitude in the left and right hemisphere ROIs for trials in which the target in the contralateral hemifield had been attended (solid line) or unattended (ipsilateral hemifield attended, dotted line). We first examined attentional modulations induced by the visual cue. Alpha amplitudes in the second following cue onset significantly differed between attention conditions in the left hemisphere ROI (Wilcoxon signed-rank test, $\mathrm{N}=17, \mathrm{p}=0.01$, mean right target attended $3.73 \mu \mathrm{V}+/-\mathrm{SD} 1.80$, mean right target unattended $3.83 \mu \mathrm{V}+/-\mathrm{SD} 1.82)$ as well as in the right hemisphere ROI (Wilcoxon signed-rank test, $N=17, p=0.01$, mean left target attended $4.42 \mu \mathrm{V}+/$ - SD 2.43, mean left target unattended $3.73 \mu \mathrm{V}+/$ - SD 1.80), showing lower alpha amplitudes in the hemisphere contralateral to the attended hemifield. We then evaluated whether this modulation persisted throughout the trial. In the second preceding RDM onset during which subjects were performing the change detection task, the effect was still present in the left hemisphere ROI (Wilcoxon signed-rank test, $\mathrm{N}=17, \mathrm{p}=0.01$, mean attended $3.15 \mu \mathrm{V}+/$ - SD 1.15, mean unattended $3.33 \mu \mathrm{V}+/$ SD 1.30). Interestingly, we no longer observed a modulation of alpha amplitude in the right hemisphere 

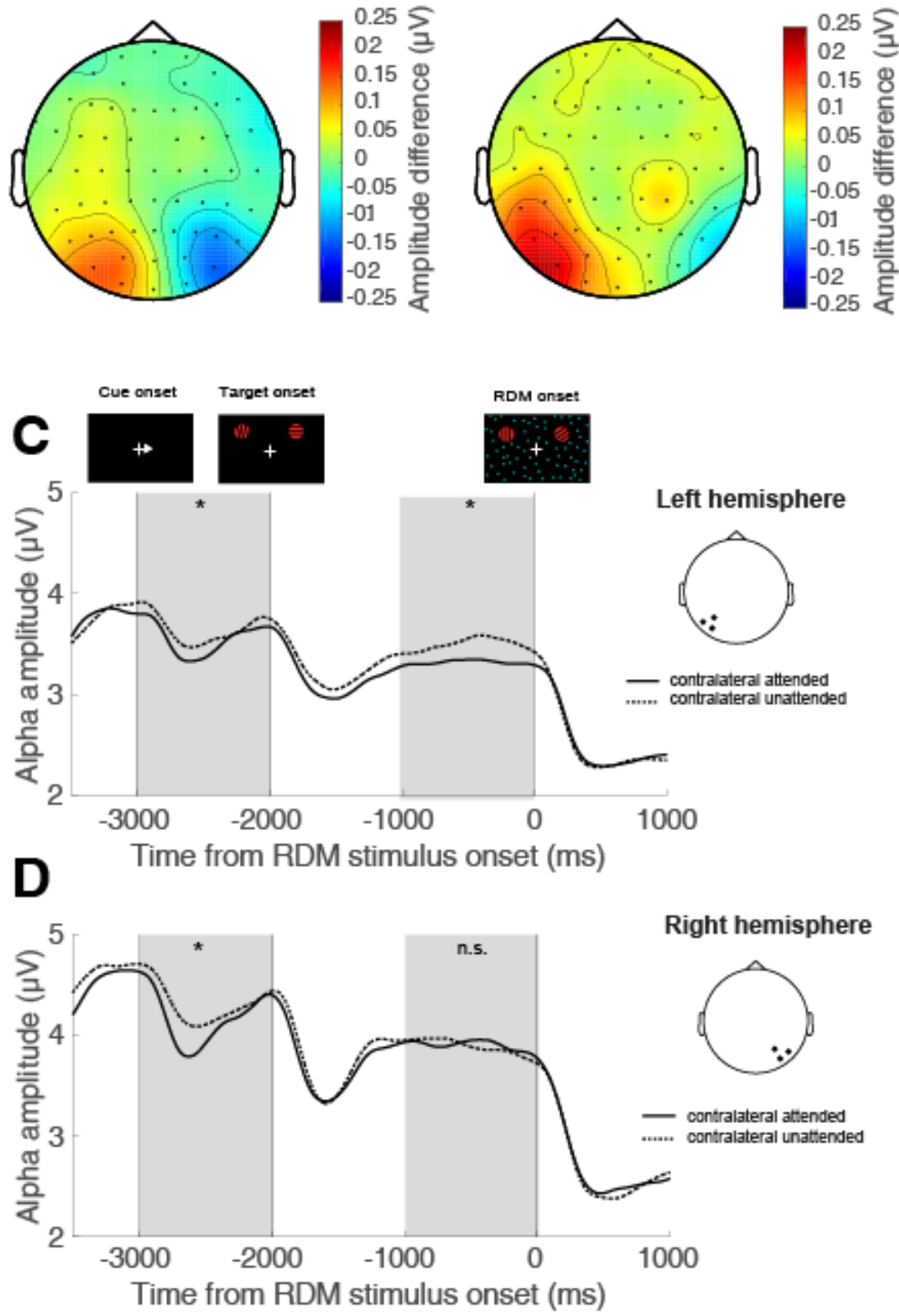

Figure 3. Main effect of spatial attention on alpha amplitude. (A) Topography of the difference in 8$12 \mathrm{~Hz}$ amplitude between attentional conditions (left attended - right attended) for the second after cue onset $(\mathrm{N}=17)$. (B) Topography of the difference in 8-12 Hz amplitude between attentional conditions (left attended - right attended) for the second preceding RDM stimulus onset $(\mathrm{N}=17)$. (C) Time courses of average $8-12 \mathrm{~Hz}$ amplitude in the left hemispheric ROI (electrodes PO7, P5, P7) over trials in which the right target was attended (solid line) and trials in which the left target was attended (dotted line, $\mathrm{N}$ $=17$ ). The post cue interval and the pre RDM stimulus interval are marked with gray shading. Asterisks indicate $\mathrm{p}<0.05$ for Wilcoxon signed-rank tests between attention conditions on the average $8-12 \mathrm{~Hz}$ amplitude in the 1 second time interval. (D) Time courses of $8-12 \mathrm{~Hz}$ amplitude as in (C) for the right hemisphere ROI (electrodes PO8, P6, P8) over trials in which the left target was attended (solid line) or unattended (dotted line, $\mathrm{N}=17$ ). 
$\mathrm{ROI}$ during the attention task (Wilcoxon signed-rank test, $\mathrm{N}=17, \mathrm{p}=0.41$, mean attended $3.78 \mu \mathrm{V}+/$ -

SD 1.57, mean unattended $3.74 \mu \mathrm{V}+/$ - SD 1.46).

\section{Perceptual modulation of alpha amplitude and factorial analysis}

Next we sorted GFS trials by whether the target stimulus contralateral to the respective hemisphere had been perceptually suppressed following the onset of the RDM stimulus (black) or had remained subjectively visible (red, Figure 4A-B). In the second prior to RDM onset, alpha amplitudes were significantly lower on trials during which the contralateral target was subsequently rendered invisible.

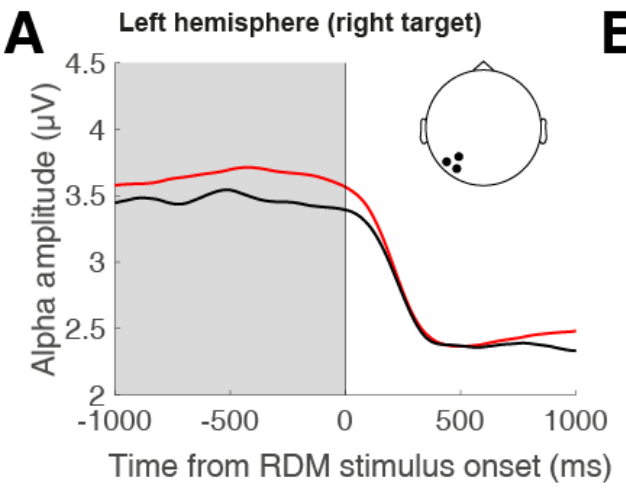

B Right hemisphere (left target)
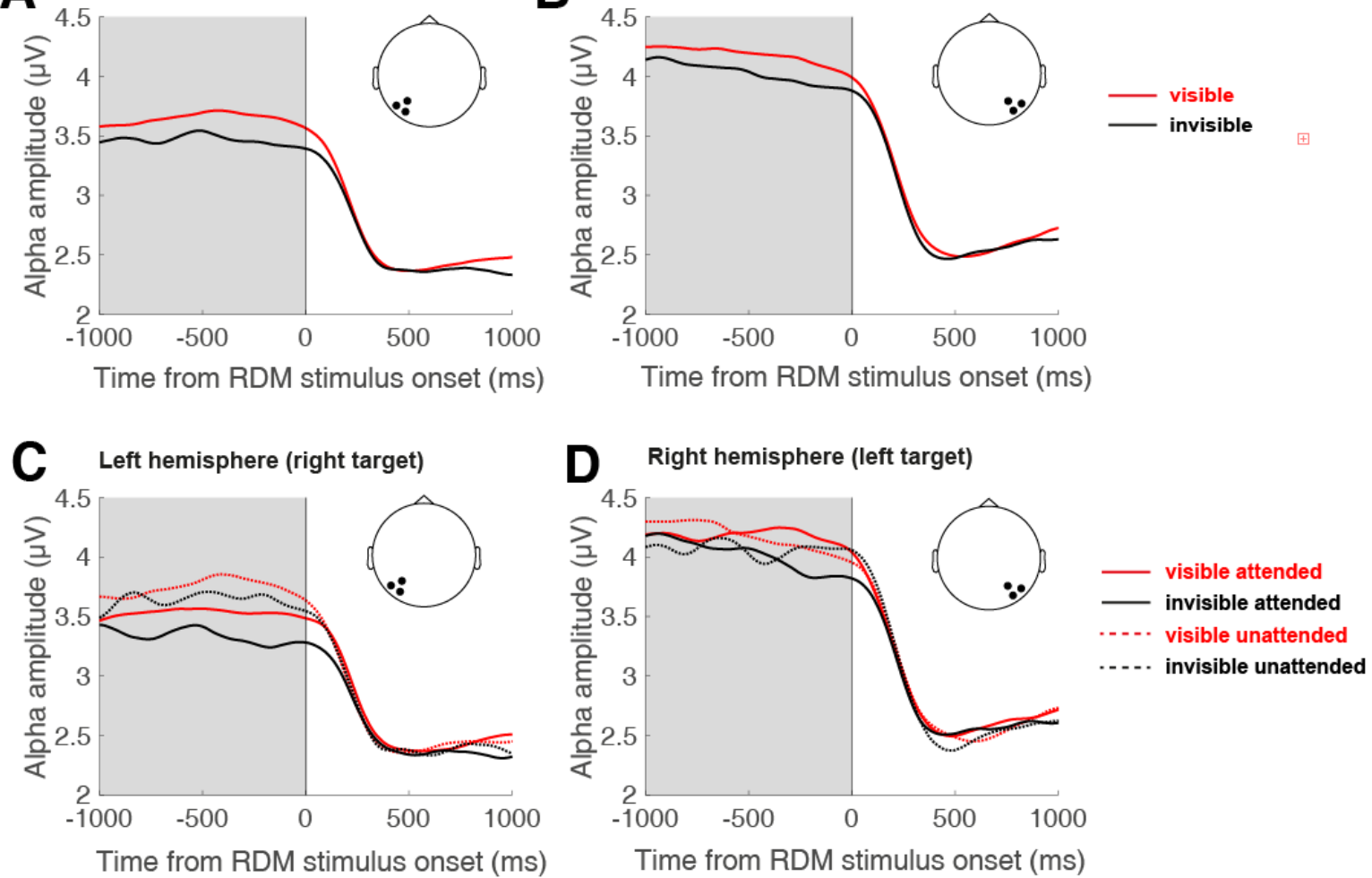

Figure 4. Main effect of perceptual suppression on alpha amplitude. (A) Time courses of average 8-12 Hz amplitude in the left hemispheric ROI across subjects for trials in which the contralateral (right) target had been perceptually suppressed (black) or remained subjectively visible (red, $N=17$ ). The shaded area illustrates the 1 second pre RDM stimulus interval used for statistical comparison.(B) Time courses of average $8-12 \mathrm{~Hz}$ amplitude in the right hemispheric ROI for trials in which the contralateral (left) target had been perceptually suppressed (black) or remained subjectively visible (red, $N=17$ ). (C) Time courses of average 8-12 Hz amplitude in the left hemisphere ROID across subjects for trials sorted by the full factorial design conditions. Solid lines indicate trials in which the contralateral (right) target was attended, dotted lines indicate trials in which the target was unattended. Red lines indicate trials in which the contralateral target remained visible, black lines indicate trials on which the target was successfully perceptually suppressed. (D) As in (C) for the right hemispheric ROI and the contralateral (left) target. 
This perceptual modulation of alpha amplitude occurred in the left hemisphere ROI (Wilcoxon signedrank test, $\mathrm{N}=17, \mathrm{p}=0.01$, mean visible $3.27 \mu \mathrm{V}+/$ - SD 1.24, mean invisible $3.13 \mu \mathrm{V}+/$ - SD 1.17), as well as in the right hemisphere ROI that had not simultaneously been modulated by the visual cueing task (Wilcoxon signed-rank test, $\mathrm{N}=17, \mathrm{p}=0.02$, mean visible $3.80 \mu \mathrm{V}+/$ - SD 1.52, mean invisible 3.66 $\mu \mathrm{V}+/$ - SD 1.49). We then further subdivided all experimental trials into a full factorial design with the factors attention and awareness for the left and right target stimulus respectively. The corresponding alpha amplitudes around the time of RDM onset are shown in Figure 4C-D. In the left hemisphere alpha amplitudes were lowest under the conditions in which the right target had been attended (solid lines), while alpha amplitudes on trials in which the targets had subsequently been rendered subjectively invisible were consistently lower than on visible trials under the same attentional conditions. Alpha amplitudes in the right hemisphere ROI which had not been significantly affected by the spatial attention task showed a less clear pattern. In order to determine whether there were any statistically significant interactions between attentional modulations of alpha amplitude and modulations of alpha amplitude predicting subsequent perceptual suppression, we performed a repeated-measures ANOVA with two within-subject factors (attention and awareness) on the average alpha amplitudes across the pre RDM stimulus interval. Average alpha amplitudes across subjects are summarized in Table 1.

\begin{tabular}{|c|c|c|}
\hline Contralateral target & Left hemisphere ROI & Right hemisphere ROI \\
\hline attended, visible & $3.53 \mu \mathrm{V}+$ - SD $1.85 \mu \mathrm{V}$ & $4.16 \mu \mathrm{V}+/$ - SD $2.15 \mu \mathrm{V}$ \\
\hline attended, invisible & $3.33 \mu \mathrm{V}+$ - SD $1.69 \mu \mathrm{V}$ & $4.01 \mu \mathrm{V}+/$ - SD $2.09 \mu \mathrm{V}$ \\
\hline unattended, visible & $3.75 \mu \mathrm{V}+$ - SD $1.99 \mu \mathrm{V}$ & $4.18 \mu \mathrm{V}+/$ - SD $2.11 \mu \mathrm{V}$ \\
\hline unattended, invisible & $3.64 \mu \mathrm{V}+/$ - SD $2.05 \mu \mathrm{V}$ & $4.07 \mu \mathrm{V}+/$ - SD $2.33 \mu \mathrm{V}$ \\
\hline
\end{tabular}

Table 1. Average alpha (8-12 Hz) amplitude in the left and right hemisphere ROls in the second preceding RDM onset for the conditions of the full factorial design with the factors attention (attended or unattended) and awareness (visible or invisible) specific to the respective contralateral target and corresponding standard deviations $(\mathrm{N}=17)$.

In the left hemisphere ROI we observed a significant main effect of attention on alpha amplitude $(F(1,16)$ $=12.22, p=0.003)$ as well as a significant main effect of subsequent awareness $F(1,16)=11.40, p=$ 0.004), but no statistically significant interaction between attention and awareness effects on alpha amplitude $(F(1,16)=2.04, p=0.17)$. In accordance with previous tests there was no significant main 
effect of attention $(F(1,16)=0.06, p=0.80)$ in the right hemisphere ROI. While we did not observe a significant main effect of awareness in the right hemisphere the difference was around the significance threshold $(F(1,16)=4.31, p=0.05)$. We did not observe a significant interaction between the factors awareness and attention in the right hemisphere $(F(1,16)=0.44, p=0.52)$.

\section{Frequencies underlying attentional and perceptual amplitude modulations}

Previous research has suggested that within the alpha frequency range, distinct sub bands can be distinguished that play different functional roles (Klimesch et al., 1998; Klimesch, 1999). We thus asked whether the modulations of parieto-occipital alpha amplitudes by spatial attention and the perceptual modulation we observed based on sorting trials by the perceptual outcome of GFS occurred at different alpha frequencies. Subjects' individual alpha frequencies (IAF) were highly similar with a mean peak frequency of $10 \mathrm{~Hz}+/-$ SD 0.9 (range $8-12 \mathrm{~Hz}$ ) and thus well captured by the $8-12 \mathrm{~Hz}$ alpha frequency range we used for statistical analysis. We then calculated the Fast Fourier Transform (FFT) for each condition separately and estimated attentional as well as perceptual modulations of alpha amplitude by calculating the absolute difference between perceptual (visible - invisible) and attentional conditions (unattended - attended) for each subject and hemisphere ROI. Figure 5 shows the mean attentional (blue) and perceptual modulation (green) across subjects relative to the IAF.

The attention task significantly modulated left hemispheric alpha amplitudes across a broad frequency range of high, low and very low alpha sub bands. The modulation of alpha amplitudes by perceptual suppression was less pronounced in lower alpha frequencies compared to the attentional modulation and more narrowly centered around the IAF, which was also the case for the perceptual modulation in the right hemisphere. We then determined the peak frequencies of perceptual as well as attentional modulation for each subject. The peak alpha frequencies for perceptual and attentional modulation relative to IAF did not statistically differ in the left hemisphere (Wilcoxon signed-rank test, $p=0.79, N=$ 17, mean perception IAF $-0.18 \mathrm{~Hz}+/-$ SD $3.26 \mathrm{~Hz}$, mean attention IAF + $0.12 \mathrm{~Hz}+/-$ SD $2.59 \mathrm{~Hz}$ ), suggesting that both effects occurred primarily around the IAF. Similarly no difference in underlying peak frequencies was observed for the right hemisphere in which attentional effects prior to RDM onset had been absent (Wilcoxon signed-rank test, $p=0.51, \mathrm{~N}=17$, mean perception IAF $-0.12 \mathrm{~Hz}+/-\mathrm{SD}$ $2.80 \mathrm{~Hz}$, mean attention IAF - $0.12 \mathrm{~Hz}+/-$ SD $4.36 \mathrm{~Hz}$ ). 

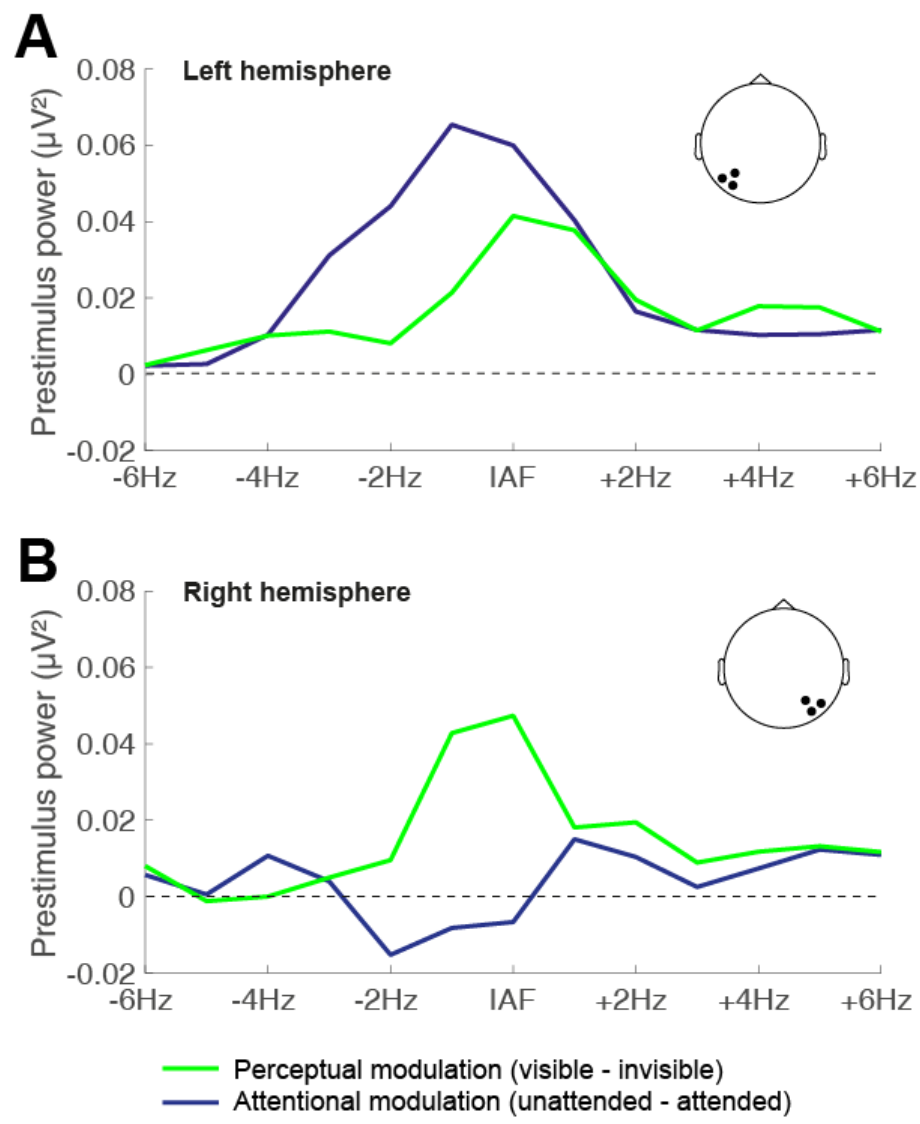

Figure 5. Low frequency range underlying the amplitude modulations by spatial attention and perceptual suppression. (A) Attentional (unattended - attended, blue) and perceptual modulations (visible - invisible, green) of the low frequency power centered around the individual alpha frequency (IAF) for the left hemisphere ROI. (B) Attentional (unattended - attended, blue) and perceptual modulations (visible - invisible, green) of the low frequency power centered around the individual alpha frequency (IAF) for the right hemisphere ROI.

\subsection{Discussion}

In the present study we manipulated subjects attention to visual target stimuli presented in the left and right hemifield that were subsequently perceptually suppressed using a GFS paradigm. Target stimuli that were attended were significantly likelier to be perceptually suppressed. Examining posterior alpha activity, we were able to replicate earlier findings (Chapter IV, unpublished) demonstrating that reported subjective target disappearances under GFS are associated with reduced parieto-occipital alpha amplitudes prior to the onset of the suppressing motion stimulus. As expected from previous research (Worden et al., 2000; Sauseng et al., 2005; Kelly et al., 2006; Thut, 2006; Wöstmann et al., 2019), our attentional manipulation resulted in decreased alpha activity in the hemisphere contralateral to the attended target. Modulations of alpha amplitude by selective attention and alpha amplitude modulations 
that predicted perceptual suppression were not separable by the affected alpha frequency range and a full factorial analysis revealed no significant interaction between the factors awareness and attention. Taken together our results suggest that alpha activity modulations that predict perceptual suppression are indistinguishable from those of anticipatory selective attention, and that the perceptual outcome of GFS on a given trial is dependent on spontaneous fluctuations in subjects' attentional state. Reduced alpha amplitudes have been suggested to reflect increased cortical excitability (Lange et al., 2013; lemi et al., 2017) and alpha activity reliably indicates the current focus of spatial attention, showing reduced alpha amplitudes corresponding to stimuli that are covertly attended when compared to unattended stimuli (Worden et al., 2000; Sauseng et al., 2005; Kelly et al., 2006; Thut, 2006). This relative difference associated with selective anticipatory attention could in theory arise from a decrease facilitating the processing of attended task-relevant information, from an increase that inhibits the processing of irrelevant distractor stimuli or from a combination of both factors, but a number of studies have reported anticipatory increases of alpha activity corresponding to locations of the visual field that contain distractor information (Kelly et al., 2006; Jensen and Mazaheri, 2010; Wöstmann et al., 2019). In the GFS paradigm, reduced alpha activity prior to RDM onset would therefor predict that the upcoming motion stimulus is processed more favorably than under low attention conditions, thus allowing it to more effectively suppress the competing target stimulus presented in the same visual hemifield. Under conditions of increased alpha activity, the visual processing of the novel RDM stimulus would instead be sufficiently hampered to allow for target information to be maintained, resulting in a stable subjective percept of the target stimulus on a different trial despite otherwise identical physical stimulation.

Our results are concordant with previous behavioural findings showing that perceptual suppression under MIB is facilitated when attention is directed to the MIB target stimuli (Geng et al., 2007; Carter et al., 2008; Schölvinck and Rees, 2009). A property common to both GFS and MIB is that both paradigms allow for the perceptual suppression of salient visual stimuli (Bonneh et al., 2001; Wilke et al., 2003) and the subjective disappearance probability of MIB targets further increases with increased target contrast (Bonneh et al., 2001), suggesting that target stimuli that draw attention facilitate perceptual suppression. When attention is instead diverted away from the MIB display entirely with a demanding perceptual task at central fixation, the probability of target disappearances instead decreases (Schölvinck and Rees, 2009). Similarly, perceptual suppression under MIB is increasingly hampered 
with increasing attentional load of a central task as well as with an increasing number of targets among which attention is to be divided (Geng et al., 2007; Carter et al., 2008), suggesting that the availability of attentional resources is a critical factor influencing the dynamics of perceptual suppression. We observe a similar effect when comparing the overall percentage of trials on which some form of perceptual suppression occurred in the present study ( $29 \%, \mathrm{~N}=30)$ with an earlier study employing the same bilateral GFS paradigm but without the addition of an attention task (67 $\%, N=27$, Chapter IV, unpublished). Both MIB and GFS require several hundred milliseconds of target adaptation which is not disrupted by changes in the target stimulus (Bonneh et al., 2001, 2014; Wilke et al., 2003, 2006) and it is thus likely that the physical stimulus changes in target orientation we employed to focus subjects attention on a given target during the SOA interval affected overall disappearance probabilities even though they did not systematically vary with the number of orientation changes.

Visual adaptation increases the relative salience of upcoming novel stimuli (McDermott et al., 2010) and MIB has been suggested to arise from a combination of local adaptation of cortical responses to the target and an additional mechanism of competition between neural populations processing target and motion stimulus respectively (Gorea and Caetta, 2009; Bonneh et al., 2014).This property conceptually links MIB to GFS and other variants of flash suppression that are similarly likely to involve competition between neuronal populations representing the static target and the motion stimulus while including additional inter-ocular conflict. When attention is directed to an adapter stimulus, perceptual afterimages brought about by visual adaptation are amplified (Yeh et al., 1996; Lou, 1999; Rhodes et al., 2011), suggesting that attention could exert a modulatory influence not only on the relative strength of the novel motion stimulus, but on the degree of target adaptation during the SOA interval.

The visual cueing paradigm and the change detection task we employed to maintain subjects' focus on either the left or the right target throughout the SOA interval likely involved a combination of spatial attention (visual hemifield) as well as feature based attention (target orientation) and may thus not have been exclusively specific to the target stimulus but extended to the motion stimulus presented in the same hemifield. Moreover, the spatial resolution of EEG recordings does not allow for a differentiation between the activity of competing neuronal populations corresponding to the target and the RDM stimulus, but we consider it likely that the net effect of alpha amplitude modulations over the left and right hemispheric ROls we measured pertain to anticipatory distractor suppression of the neuronal 
populations processing the upcoming motion stimulus. Our aim in using a visual cueing paradigm was to decrease alpha activity in the hemisphere contralateral to the respective attended target during the SOA interval of target presentation prior to the onset of the RDM pattern, which we hypothesized would affect subsequent GFS disappearance probabilities. Interestingly only the overall attentional modulation of alpha activity in the left hemisphere persisted during the SOA interval, while disappearance probabilities were higher for attended right as well as for attended left targets. This discrepancy is in line with theories suggesting a hemispheric asymmetry in coordinating spatial attention. More specifically, alpha desynchronization in the left hemisphere has been shown to occur primarily in response to visual stimuli in the contralateral visual hemifield, while right-hemispheric desynchronization was equally strong in response to stimuli in the contralateral and ipsilateral visual field, suggesting a right-hemispheric dominance for attention (Heilman and Van Den Abell, 1980). The absence of a measurable overall difference between attentional conditions in the right hemisphere could thus be attributed to opposing effects pertaining to the left and right hemifield. A recent study investigating left and right hemispheric contributions separately showed that, consistent with the present findings, posterior alpha activity in the left hemisphere was generally lower and more strongly modulated than in the right hemisphere (Gallotto et al., 2020). The authors further demonstrated that left hemispheric alpha activity was only modulated rightward shifts in attention directed by a visual cue, while right hemispheric alpha activity was modulated for both leftward and rightward shifts (Gallotto et al., 2020). Based on the present findings we can conclude that perceptual suppression is dependent on attentional state prior to the onset of the motion stimulus, and that predictive modulations of alpha activity likely reflect anticipatory distractor suppression pertaining to the portions of the visual field in which the novel motion stimulus is expected. The strength of inhibitory attentional modulation in turn influences the relative strength of the upcoming motion stimulus and its effectiveness in perceptually suppressing the target, but likely does not indicate visual awareness of the target stimulus as such. 


\subsection{References}

Benwell CSY, Tagliabue CF, Veniero D, Cecere R, Savazzi S, Thut G (2017) Prestimulus EEG Power Predicts Conscious Awareness But Not Objective Visual Performance. eNeuro 4 Available at: https://www.ncbi.nlm.nih.gov/pmc/articles/PMC5732016/ [Accessed April 29, 2019].

Bonneh YS, Cooperman A, Sagi D (2001) Motion-induced blindness in normal observers. Nature 411:798-801.

Bonneh YS, Donner TH, Cooperman A, Heeger DJ, Sagi D (2014) Motion-Induced Blindness and Troxler Fading: Common and Different Mechanisms Lu Z-L, ed. PLoS ONE 9:e92894.

Brainard DH (1997) The Psychophysics Toolbox. Spat Vis 10:433-436.

Carter O, Luedeman R, Mitroff S, Nakayama K (2008) Motion induced blindness: The more you attend the less you see. Journal of Vision 8:237-237.

Dijk H van, Schoffelen J-M, Oostenveld R, Jensen O (2008) Prestimulus Oscillatory Activity in the Alpha Band Predicts Visual Discrimination Ability. J Neurosci 28:1816-1823.

Ergenoglu T, Demiralp T, Bayraktaroglu Z, Ergen M, Beydagi H, Uresin Y (2004) Alpha rhythm of the EEG modulates visual detection performance in humans. Cognitive Brain Research 20:376-383.

Gallotto S, Duecker F, Oever S ten, Schuhmann T, de Graaf TA, Sack AT (2020) Relating alpha power modulations to competing visuospatial attention theories. Neurolmage 207:116429.

Geng H, Song Q, Li Y, Xu S, Zhu Y (2007) Attentional modulation of motion-induced blindness. Chin Sci Bull 52:1063-1070.

Gorea A, Caetta F (2009) Adaptation and prolonged inhibition as a main cause of motion-induced blindness. Journal of Vision 9:16-16.

Hanslmayr S, Aslan A, Staudigl T, Klimesch W, Herrmann CS, Bäuml K-H (2007) Prestimulus oscillations predict visual perception performance between and within subjects. Neurolmage 37:1465-1473.

Heilman KM, Van Den Abell T (1980) Right hemisphere dominance for attention: the mechanism underlying hemispheric asymmetries of inattention (neglect). Neurology 30:327-330.

lemi L, Chaumon M, Crouzet SM, Busch NA (2017) Spontaneous Neural Oscillations Bias Perception by Modulating Baseline Excitability. J Neurosci 37:807-819.

Jensen O, Mazaheri A (2010) Shaping Functional Architecture by Oscillatory Alpha Activity: Gating by Inhibition. Frontiers in Human Neuroscience 4 Available at: http://journal.frontiersin.org/article/10.3389/fnhum.2010.00186/abstract [Accessed July 25, 2019].

Kelly SP, Lalor EC, Reilly RB, Foxe JJ (2006) Increases in Alpha Oscillatory Power Reflect an Active Retinotopic Mechanism for Distracter Suppression During Sustained Visuospatial Attention. Journal of Neurophysiology 95:3844-3851.

Kim C-Y, Blake R (2005) Psychophysical magic: rendering the visible 'invisible.' Trends in Cognitive Sciences 9:381-388.

Klimesch W (1999) EEG alpha and theta oscillations reflect cognitive and memory performance: a review and analysis. Brain Research Reviews 29:169-195.

Klimesch W, Doppelmayr M, Russegger H, Pachinger T, Schwaiger J (1998) Induced alpha band power changes in the human EEG and attention. Neuroscience Letters 244:73-76.

Koch C, Tsuchiya N (2007) Attention and consciousness: two distinct brain processes. Trends in Cognitive Sciences 11:16-22. 
Lamme VAF (2003) Why visual attention and awareness are different. Trends in Cognitive Sciences 7:12-18.

Lange J, Oostenveld R, Fries P (2013) Reduced Occipital Alpha Power Indexes Enhanced Excitability Rather than Improved Visual Perception. J Neurosci 33:3212-3220.

Lou L (1999) Selective peripheral fading: evidence for inhibitory sensory effect of attention. Perception 28:519-526.

Macdonald JSP, Mathan S, Yeung N (2011) Trial-by-Trial Variations in Subjective Attentional State are Reflected in Ongoing Prestimulus EEG Alpha Oscillations. Front Psychol 2 Available at: https://www.frontiersin.org/articles/10.3389/fpsyg.2011.00082/full [Accessed April 29, 2019].

Oostenveld R, Fries P, Maris E, Schoffelen J-M (2011) FieldTrip: Open Source Software for Advanced Analysis of MEG, EEG, and Invasive Electrophysiological Data. Computational Intelligence and Neuroscience 2011:1-9.

Rhodes G, Jeffery L, Evangelista E, Ewing L, Peters M, Taylor L (2011) Enhanced attention amplifies face adaptation. Vision Research 51:1811-1819.

Sauseng P, Klimesch W, Stadler W, Schabus M, Doppelmayr M, Hanslmayr S, Gruber WR, Birbaumer N (2005) A shift of visual spatial attention is selectively associated with human EEG alpha activity. Eur J Neurosci 22:2917-2926.

Schölvinck ML, Rees G (2009) Attentional influences on the dynamics of motion-induced blindness. Journal of Vision 9:38-38.

Thut G (2006) -Band Electroencephalographic Activity over Occipital Cortex Indexes Visuospatial Attention Bias and Predicts Visual Target Detection. Journal of Neuroscience 26:9494-9502.

Wilke M, Logothetis NK, Leopold DA (2003) Generalized Flash Suppression of Salient Visual Targets. Neuron 39:1043-1052.

Wilke M, Logothetis NK, Leopold DA (2006) Local field potential reflects perceptual suppression in monkey visual cortex. PNAS 103:17507-17512.

Worden MS, Foxe JJ, Wang N, Simpson GV (2000) Anticipatory biasing of visuospatial attention indexed by retinotopically specific alpha-band electroencephalography increases over occipital cortex. J Neurosci 20:RC63.

Wöstmann M, Alavash M, Obleser J (2019) Alpha Oscillations in the Human Brain Implement Distractor Suppression Independent of Target Selection. J Neurosci 39:9797-9805.

Yeh T, Lee BB, Kremers J (1996) The time course of adaptation in macaque retinal ganglion cells. Vision Research 36:913-931. 


\section{Chapter VI \\ General Discussion}

\subsection{Neural mechanisms of generalized flash suppression (GFS)}

\subsubsection{Conceptual background and summary of the present findings}

In its original description, generalized flash suppression (GFS) has been suggested to arise from a combination of several contributing factors that conjointly result in a salient visual target stimulus being erased from visual awareness in an all-or-none fashion: Target adaptation, surround inhibition and interocular conflict (Wilke et al., 2003). In a set of psychophysical experiments, Wilke and colleagues demonstrated that perceptual suppression under GFS is dependent on several hundred milliseconds of target adaptation prior to the onset of the suppressing motion stimulus and considerably hampered when target adaptation is disrupted by changes in target position or orientation (Wilke et al., 2003), a requirement GFS shares with a related visual illusion, motion-induced blindness (MIB) (Bonneh et al., 2001). In addition to visual adaptation, another mechanism that may contribute to perceptual disappearances under GFS is surround inhibition, as suggested by the fact that the effectiveness of GFS is influenced by the distance between the target stimulus and the spatially non-overlapping motion stimulus (Wilke et al., 2003). Neighbouring visual stimuli can interfere with the visibility of a central stimulus, effectively inhibiting its perception (He et al., 1996), and it is possible that even in the absence of direct local conflict perceptual suppression arises from neural competition between representations of the target and the motion stimulus respectively, which has been suggested to similarly occur under MIB (Gorea and Caetta, 2009; Bonneh et al., 2014). A third critical factor determining the effectiveness of GFS, which it does not share with MIB but with other variants of flash suppression, is inter-ocular conflict as perceptual suppression is largely absent when target and RDM stimulus are presented to the same eyes (Wilke et al., 2003). By itself, a mismatch between the visual information received from different eyes phenomenologically results in spontaneous alternations between the perception of either one or the other stimulus rather than in a superimposed percept, a phenomenon known as binocular rivalry (Wolfe, 1984). Based on fMRI experiments demonstrating opposing activations in brain regions 
specific to the two competing stimuli under binocular rivalry which alternate depending on the currently perceived stimulus (Tong et al., 1998), it is likely that the inter-ocular conflict between target and motion stimulus during GFS adds another layer of neural competition that hampers the integration of both stimuli into a consistent conscious percept.

In the present dissertation, we showed that attention directed to a GFS target stimulus with a visual cueing paradigm and a subsequent change detection task increases its probability of subjective disappearance relative to the unattended target (Chapter V). This effect is associated with reduced parieto-occipital alpha activity prior to successful perceptual suppression compared to physically identical trials in which the conscious percept of the target was not perturbed by the onset of the motion stimulus (Chapter IV, Chapter V), which has previously been linked to increased selective anticipatory attention (Worden et al., 2000; Sauseng et al., 2005a; Kelly et al., 2006; Thut, 2006; Rihs et al., 2007; Wöstmann et al., 2019). Consistent with the psychophysical results and the examination of posterior alpha activity in the human encephalogram (EEG), an analysis of electrophysiological data recorded from visual area V4 of two rhesus macaques showed effects of perceptual suppression on neuronal firing rates and variability (Chapter III) that strongly resemble those previously reported for attentional modulation in V4 (Mitchell et al., 2007, 2009; Cohen and Maunsell, 2009). Taken together, the studies presented here demonstrate that the dynamics of GFS are dependent on attentional state.

\subsubsection{Attentional influences on local target adaptation}

How does attention increase the effectiveness of perceptual suppression? In theory there are several ways in which attentional modulation could exert an influence on the perception of the target stimulus. The first possible interpretation would be that attention affects target adaptation. Prolonged visual stimulation can be expected to result in a decreased responsiveness of neural populations encoding the adapter stimulus, that could hamper the continued integration of feedforward stimulus representations with other processing stages of the visual system while neurons encoding the adapter stimulus are in a hyperpolarized state (Carandini and Ferster, 1997; Carandini et al., 1998; Wilke et al., 2003). Adapter stimuli that are attended produce stronger perceptual afterimages than adapter stimuli that are unattended (Yeh et al., 1996; Lou, 1999; Rhodes et al., 2011), suggesting that increased 
attention amplifies visual adaptation. Selective attention is typically conceptualized as boosting the relative strength of a specific stimulus and a study investigating the effect of sustained attention on perception found that, in line with this interpretation, attention initially increases contrast sensitivity (Ling and Carrasco, 2006). However, this effect only persisted for the first 1-2 seconds of stimulus presentation until it reversed to lower contrast sensitivity under attention over time, suggesting that the strengthening of neural representations by attention leads to stronger adaptation (Ling and Carrasco, 2006). In the context of GFS, an amplification of the neural signals representing one of the target stimuli by attention could thus exacerbate adaptation and lead to weakened low-level target representations. We presented the target stimuli with a stimulus onset asynchrony (SOA) of 2 seconds before presenting the perceptual suppression inducing motion stimulus, making it probable that the effect of attention on target salience would have been negative at motion onset. Adaptation to colour has been shown to affect the relative salience of colour stimuli as assessed by improved stimulus detection, suggesting that visual adaptation allows for discounting of ambient features in a visual display while highlighting novel stimuli (McDermott et al., 2010). It could thus be argued that increased attention directed to a GFS target results in stronger adaptation and more substantially weakens low-level neural representations of the target, making it likelier to be discarded when a stronger incongruent stimulus is presented. Equivalent effects of attention on the probability of target disappearances reported for MIB (Geng et al., 2007; Carter et al., 2008; Schölvinck and Rees, 2009) and its sensitivity to target adaptation (Bonneh et al., 2001) suggest that the same principle may apply to both phenomena.

\subsubsection{Attentional influences on stimulus competition}

Besides local target adaptation, attentional modulations of neural activity could also affect higher level sensory selection mechanisms that involve neural representations of competing visual stimuli such as the static target and the motion stimulus during GFS. This interpretation is consistent with electrophysiological findings demonstrating that spiking activity in early visual cortex areas V1/V2 does not reliably reflect the perceptual outcome of GFS, whereas responses in extrastriate visual area V4 are considerably modulated during perceptual suppression (Wilke et al., 2006; Chapter III). Attentional selection mechanisms have also been proposed to underlie MIB based on a study showing that 
transcranial magnetic stimulation (TMS) pulses suppressing parietal activity either improved or hampered perceptual suppression under MIB depending on the stimulated hemisphere (Bonneh et al., 2001; Funk and Pettigrew, 2003). Pathologies of perception observed in simultanagnosia patients who experience difficulties interpreting visual arrays of multiple stimuli and report alternating disappearances of objects following bilateral parieto-occipital lesions similarly support the hypothesis that perceptual suppression may involve higher level attentional mechanisms (Rizzo and Robin, 1990; Coslett and Saffran, 1991; Bonneh et al., 2001). An fMRI study in humans investigating the neural basis of MIB provides additional evidence for this account: Target-specific activity in ventral stream visual area V4 decreased during MIB target disappearances while activity in motion-specific dorsal area V5/MT corresponding to the suppressing motion stimulus simultaneously increased (Donner et al., 2008). This finding in particular would argue for neural competition between the two stimuli that, during subjective target disappearances, results in dorsal representations of the motion stimulus taking over in a winner takes all fashion.

\subsubsection{Modulations of neural activity during perceptual suppression}

Neural correlates of perceptual suppression obtained from spiking activity and fMRI BOLD responses have been shown to markedly diverge despite being measured with the same subjects and stimuli (Maier et al., 2008), which complicates the comparison between such fMRI findings and our electrophysiological results in V4. We found V4 spiking activity during perceptual suppression to be significantly increased (Chapter III), but the directionality of the firing rate modulations in area V4 has been shown to depend on the directionality of the sites responsiveness to physical target removal (Wilke et al., 2006) and it is likely that the increase we observed across the examined population was due to the majority of sites responding with rate increases to target removal, which may not be representative of the overall net effect in V4 (Chapter III). Unfortunately we were not able to differentiate between neural representations of the target and the motion stimulus in the examined V4 data set as the majority of recording sites were responsive to both stimuli (Chapter III), which constitutes a major limitation of the present studies. Increased V4 firing rates and decreased V4 spiking variability have been observed during the sustained response to visual stimuli that were attended compared to stimuli that were 
unattended (Mitchell et al., 2007, 2009; Cohen and Maunsell, 2009), suggesting that the equivalent modulations we observed during perceptual suppression compared to trials in which the target was not suppressed reflect increased attention during perceptual suppression (Chapter III). Whether these modulations are an indication of attention towards the target or towards the motion stimulus is however not a trivial question to answer. One possible perspective is that V4 activity is generally likelier to correspond to the static red target stimulus rather than to visual motion and that the modulations we observed represent increased attention towards the target, which counterintuitively increases its probability of disappearance due to stronger visual adaptation that degrades lower level target representations as argued earlier. Conscious perception has been hypothesized to arise from recurrent activity between different stages of the sensory processing stream in addition to feedforward activity (Lamme, 2003), and it is possible that even though target representations in V4 are amplified by attention, neural representations of the target in earlier visual areas are too extensively weakened to be integrated into a unified conscious percept once the motion stimulus is added. This hypothesis is supported by findings of significantly reduced BOLD responses in early visual cortices during perceptual suppression of the target under GFS, which are however not confirmed by examinations of V1/V2 spiking activity (Wilke et al., 2006; Maier et al., 2008). Conversely, the results presented in Chapter III may also reflect increased attention towards the appearing motion stimulus to which the examined neuronal responses corresponded. This interpretation would be consistent with an amplification of neural signals representing the RDM stimulus that win over during perceptual suppression of the target.

\subsubsection{Modulations of neural activity predicting perceptual suppression}

Interestingly we only observed modulations of posterior alpha activity prior to perceptual suppression in the human EEG (Chapter IV, Chapter V), while such predictive modulations were absent in the examined local field potential (LFP) data recorded from macaque V4 (Chapter III). In Chapter IV we compared alpha activity between bilateral target disappearances with trials in which both targets were perceived across visual cortex, in Chapter $\vee$ we additionally directed subjects' attention to one hemifield target and focused on alpha activity in the hemisphere contralateral to a given target. Alpha lateralization, that is a hemispheric asymmetry in the relative strength of posterior alpha oscillations, has been shown to reliably indicate the current focus of covert spatial attention towards stimuli in the left or in the right visual hemifield, and it also differentiates between attention towards the upper and 
lower quadrant of the same visual hemifield (Worden et al., 2000; Thut, 2006; Ikkai et al., 2016). It is thus possible that during selective attention, portions of the visual field that are likely to contain taskirrelevant distractor information are suppressed by a stronger alpha rhythm in a relatively retinotopically specific manner. The spatial resolution of EEG recordings of the sum potential of all cortical neurons in sufficient proximity to the detecting electrodes does however not allow for a more precise separation between modulations specific to the target and the motion stimulus presented within the same hemifield, resulting in the same interpretation predicament as for the electrophysiological results. Based on the conceptualization of alpha activity as a sensory suppression mechanism that selectively reduces cortical excitability for efficient distractor suppression it could be argued that the overall net effect of reduced posterior alpha activity we measured prior to perceptual suppression reflects reduced attention towards the target stimulus itself. Increased attention towards the target specifically could increase anticipatory distractor suppression for the largest surface area of the visual field wherein the competing RDM stimulus is expected to appear, which would result in an overall increase in alpha activity in the same hemisphere even though the target is attended. When distractor suppression of the surrounding visual field is less efficient, contralateral alpha amplitudes would be lower and the upcoming motion stimulus would be more effectively processed, leading to subjective target invisibility. Do the attentional modulations we observed in Chapter V support this hypothesis? When a given target was attended we observed reduced alpha amplitudes in the contralateral hemisphere. However, this interpretation of the results does not take into account the respective ignored visual hemifield and it is possible that the attentional modulations of posterior alpha power that tend to divide between the hemispheres are more pronounced than those pertaining to competing stimuli within the same hemifield. Increased attention towards one hemifield target might thus result in diminished suppression of the motion stimulus within the same visual hemifield, rendering it more effective in perceptually suppressing the target stimulus. 


\subsection{Alpha oscillations and neuronal variability in visual awareness}

\subsubsection{Conceptual background and summary of the present findings}

An examination of the largely separate literatures on neural oscillations in the alpha band and neuronal variability across trials reveals many parallels between the two measures of brain activity. Neural variability substantially decreases with the onset of a visual stimulus (Churchland et al., 2010), as does the amplitude of posterior alpha oscillations, a phenomenon known as event-related desynchronization (ERD) (Pfurtscheller and Lopes da Silva, 1999). The degree to which neural variability in human EEG recordings is quenched by visual stimulation is a stable individual characteristic that determines individual perceptual thresholds (Arazi et al., 2017b, 2017a), while individual perceptual performance is also predicted by the individual level of parieto-occipital alpha activity prior to stimulus onset (Hanslmayr et al., 2007). The detection of visual stimuli presented at the perceptual threshold is generally associated with reduced trial-to-trial variability (Ress et al., 2000; Schurger et al., 2015) as well as reduced alpha amplitudes (Ergenoglu et al., 2004; Hanslmayr et al., 2007; Dijk et al., 2008; Benwell et al., 2017), and both neural variability across trials and alpha amplitudes are reduced for stimuli that are attended compared to unattended stimuli (Sauseng et al., 2005b; Kelly et al., 2006; Thut, 2006; Mitchell et al., 2007, 2009; Cohen and Maunsell, 2009; Mo et al., 2011; Arazi et al., 2019). Based on these striking similarities between the functional roles of neural variability and alpha oscillations in visual perception it stands to reason that the amount of trial-to-trial variability typically measured in neuronal spiking activity could at least partially be explained by the strength of lowfrequency cortical oscillations.

In the dissertation presented here we showed that neural spiking activity in the visual thalamus, more specifically the lateral geniculate nucleus (LGN) and the dorsal and ventral pulvinar, does not exhibit reductions in variability upon stimulus onset to the same degree as in visual cortex area V4 examined in the same animals (Chapter II). Moreover, we found ongoing thalamic activity prior to stimulus onset to be considerably less variable than cortical activity (Chapter II), suggesting a dependence of the decrease on the amount of variability inherent in the spontaneous neural activity preceding visual stimulation. Investigating the effects of perceptual suppression on neural variability as well as on the amplitude of the simultaneously recorded local field potential (LFP) in macaque visual area V4, we 
found a significant reduction in neural variability around the time of perceptual suppression that coincided with a significant reduction in the amplitude of LFP low-frequency oscillations around the alpha range (Chapter III). A comparison of alpha amplitude and spiking variability in the examined time window showed a positive site-by-site correlation between the two measures (Chapter III). In the human EEG, we found the individual degree of variability quenching to be closely correlated with the degree of individual reductions of alpha amplitude with stimulus onset (Chapter IV). Interestingly, both stimulusinduced decreases were also dependent on the amplitude of alpha band oscillations prior to stimulus onset and examining time courses of the raw variance across trials and alpha amplitude of individual subjects we noted that both measures closely co-varied (Chapter IV). The results presented here thus suggest two main conclusions which will be discussed in more detail in the following sections: 1) The amount of neural variability across trials largely reflects the amplitude of low-frequency oscillations, and 2) Their respective stimulus-induced decreases are dependent on the spontaneous activity dynamics preceding them.

\subsubsection{On the relationship between alpha activity and neural variability}

Neural variability is thought to primarily arise from ongoing fluctuations in cortical excitability that are highly correlated between large populations of neurons (Arieli et al., 1996; Goris et al., 2014; Schölvinck et al., 2015) (Arieli et al., 1996; Goris et al., 2014; Schölvinck et al., 2015). The magnitude of neural variability thus depends on cortical state, which likely exhibits a continuum ranging from highly synchronized states that are characterized by strong low-frequency oscillations, to more desynchronized states during which such activity fluctuations are largely absent (Harris and Thiele, 2011; Schölvinck et al., 2015). Neural oscillations in the alpha band specifically have been intensively studied and shown to have an inverse relationship to cortical excitability (Lange et al., 2013; lemi et al., 2017; Samaha et al., 2017). Despite these functional parallels between alpha activity and neural variability, which further both vary with visual perception and attentional states in a similar manner, the two neural signatures have largely been investigated and reported independently. One recent study finally conclusively linked trial-to-trial variability and low- frequency oscillations: Daniel and colleagues could show that the two measures of brain activity are tightly coupled and that on the individual level the largest portion of inter subject differences was explained by individual power in the alpha and beta frequency range (Daniel et al., 2019). When the data were filtered with a band-stop procedure removing 
the influence of a specific frequency band on the data, variability changes with stimulus onset were unaffected by the removal of the delta and gamma frequency bands, while neural variability increased relative to prestimulus baseline in the absence of alpha/beta frequency oscillations (Daniel et al., 2019). These results are largely consistent with our examination of frequency contributions to variability quenching in the human EEG (Chapter IV, Supplementary information S3). Daniel and colleagues considered a frequency range spanning $5-25 \mathrm{~Hz}$ as the alpha/beta band since they did not observe any qualitative differences when separating alpha and beta frequencies (Daniel et al., 2019). This range partially includes theta $(4-7 \mathrm{~Hz})$, alpha $(8-12 \mathrm{~Hz})$ and beta $(13-30 \mathrm{~Hz})$ frequencies, which we analyzed separately. We similarly found the impact of removing theta and gamma oscillations on variability quenching to be minimal, while considerably less variability quenching occurred without the alpha range (Chapter IV, Supplementary information S3). Band-stop filtering in the beta range did not affect variability quenching in our case (Chapter IV, Supplementary information S3). However, unlike Daniel et al. we also examined the absolute variance rather than the relative change in variance from prestimulus baseline and found that removing the influence of beta oscillations reduced both prestimulus variance as well as poststimulus variance to the same degree (Chapter IV, Supplementary information S3, Figure 2A). Prestimulus variance was most profoundly reduced following band-stop filtering in the alpha $(8-12 \mathrm{~Hz})$ range, resulting in a minimal stimulus-induced decrease to the same level of poststimulus variance observed for the removal of beta oscillations (Chapter IV, Supplementary information S3, Figure S2A). We also observed qualitatively similar changes in trial-to-trial spiking variability as well as simultaneously recorded theta, alpha and beta oscillations of the monkey LFP related to perceptual suppression (Chapter III). It is thus likely that while alpha oscillations, which are characteristically higher in amplitude than those in other frequency bands (Chapter IV, Supplementary information S3, Figure S2B), constitute the largest contribution to neural variability, this relationship is not exclusive to the classical alpha band. Concordantly, a recent study examining spiking activity related to movement execution in behaving macaques found reductions in trial-to-trial spiking variability to correlate with reductions in LFP beta power (Riehle et al., 2018).

The decline of trial-to-trial variability with stimulus onset could in theory also be explained by increased phase-locking of low-frequency oscillations rather than by a decrease in their amplitude, which we did not control for in our studies. Daniel et al. were however able to conclusively rule out this alternative explanation as they found inter-trial phase coherence to be uncorrelated with neural variability 
quenching, suggesting that reductions in neural variability are in fact caused by reductions in oscillatory power (Daniel et al., 2019). Moreover, they found the spatial topographies of neural variability quenching and the ERD in the alpha/beta range to closely match and to be centered around the parieto-occipital areas we considered for our analyses (Daniel et al., 2019) (Chapter IV), further confirming the direct relationship between the two measures.

\subsubsection{Are stimulus-induced changes determined by ongoing activity?}

In the present dissertation we observed a strong correlation between individual prestimulus alpha amplitude and the degree to which neural variability across trials as well as posterior alpha amplitude declined with RDM stimulus onset, suggesting that the previously examined ERD in the alpha band as well as neural variability quenching may depend on the level of prestimulus alpha activity (Chapter IV). The hypothesis is further supported by the finding that the removal of low-frequency oscillations in the alpha and to some degree in the beta range resulted in considerable reductions in prestimulus variance while poststimulus variance was only minimally affected (Chapter IV, Supplementary information S3, Figure 2A). Stimulus-induced decreases of neural variability have consistently been observed across various measures of brain activity including the membrane potential of cats (Churchland et al., 2010), spiking activity of non-human primates (Churchland et al., 2006, 2010; Oram, 2011; Poland et al., 2019) (Chapter II), human EEG / MEG recordings (Arazi et al., 2017b, 2017a, 2019) (Chapter IV) and the human fMRI (Broday-Dvir et al., 2018). The phenomenon of neural variability quenching is generally thought of as a network effect that arises from large neuronal populations independent of their responsiveness and stimulus specificity (Churchland et al., 2010; Rajan et al., 2010; Deco and Hugues, 2012; Goris et al., 2014) and improves the reliability of sensory processing (Zohary et al., 1994; Shadlen et al., 1996; Parker and Newsome, 1998). Here we showed that neural variability quenching does not occur to the same degree in the LGN and higher-order thalamus as in simultaneously recorded visual cortex V4, while spontaneous thalamic spiking activity prior to stimulus onset was already considerably more reliable than cortical activity (Poland et al., 2019) (Chapter II). This finding further hints at a possible dependency of ERD and variability quenching on the magnitude of ongoing activity fluctuations preceding them. 
Previous studies have highlighted the role of ongoing activity in sensory processing, suggesting that evoked activity constitutes a linear summation of a deterministic stimulus response onto ongoing activity fluctuations prior to stimulus onset, which explain the large degree of variability in neural responses and its dependence on cortical state (Arieli et al., 1996; Schölvinck et al., 2015). Moreover, selective attention is observed to coincide with a desynchronization, which is a decrease in the amplitude of lowfrequency oscillations, occurring in cortical regions that process the attended stimulus (Harris and Thiele, 2011). Similar desynchronizations of low frequency oscillations occur following external stimulus input and largely explain the phenomenon of variability quenching (Daniel et al., 2019). It could thus be argued that stimulus-induced changes in cortical state are dependent on the degree to which anticipatory attention is assigned to a given stimulus, which is conceptually supported by previous observations of larger magnitudes of variability quenching in response to attended compared to unattended stimuli (Arazi et al., 2019). A previous EEG study found only the relative variance, which is the amount by which variability decreases with stimulus onset, to differentiate between perceptual outcomes, while the absolute variance post stimulus was not perceptually relevant (Arazi et al., 2017a). While the interpretation of negative results has to be approached with caution, this surprising finding could in theory be attributed to significant differences in prestimulus alpha amplitude brought about by anticipatory attention, which were not specifically examined within the same study and previously shown to predict subsequent stimulus perception (Chapter IV; Chapter V). Many previous studies have primarily considered relative changes with stimulus onset (Churchland et al., 2010; Arazi et al., 2017b; Daniel et al., 2019), making it difficult to determine whether the improved perceptual performance associated with stronger variability quenching and ERD is mechanistically determined by neural activity dynamics prior to or following stimulus onset, which may further be strongly correlated. In order to impact sensory perception on a given trial, neuronal spiking variability would have to be apparent not only across trials but also in the temporal pattern present during stimulus processing, and stronger variability quenching has previously been associated with increased pattern stability within-trial (Schurger et al., 2015). Our findings comparing trial-to-trial variability between thalamus and cortex suggest that the magnitude by which variability quenches with stimulus onset is restricted by the degree to which the reliability of spiking responses can be further improved, resulting in reduced variability quenching in the thalamus wherein we found Fano factors of ongoing activity to already be close to 1 and thus close to the amount of variability expected to be inherent in neuronal spiking without the 
additional impact of correlated activity fluctuations (Poland et al., 2019) (Chapter II). In a combined EEG-fMRI study, Becker and colleagues were able to show that the variability in evoked responses in the human fMRI is largely explained by ongoing alpha activity, which further strongly supports our hypothesis that the power of low-frequency oscillations preceding stimulus onset determines the reliability of subsequent stimulus processing (Becker et al., 2011). Increased cortical excitability associated with reductions in low frequency power as well as reduced variability following visual stimulation are conceptually likely to considerably improve stimulus processing, and the correlation between prestimulus alpha amplitude and ERD in the alpha band as well as neural variability quenching we observed (Chapter IV) hint at such dynamics underlying a common neural mechanism of anticipatory selective attention.

\subsection{Conclusions}

In the present dissertation we could show that the generalized flash suppression (GFS) paradigm is dependent on attentional state and that attention directed to a GFS target stimulus increases its probability of disappearance relative to the other target for both visual hemifields (Chapter V). This behavioural finding can be explained by stronger local target adaptation under attention, as well as by effects of attentional mechanisms on neural competition between the target and the motion stimulus. Attention to a GFS target was associated with reduced posterior alpha amplitudes prior to motion onset in the contralateral hemisphere, which is likely to increase the relative processing strength of the upcoming motion stimulus presented in the same visual hemifield, thus rendering it more effective in suppressing the competing static target from awareness. Consistent with this view, we observed that perceptual suppression under GFS was predicted by reduced parieto-occipital alpha amplitudes both under conditions in which attention was directed by a visual cueing paradigm (Chapter V) and under conditions in which attention was not externally manipulated (Chapter IV). An examination of spiking activity recorded from macaque visual cortex area V4 showed modulations of firing rates and trial-totrial spiking variability associated with perceptual suppression that are consistent with modulations observed for selective attention (Chapter III). These findings link the neural mechanisms underlying GFS to a similar visual illusion, motion-induced blindness (MIB). The reductions of spiking variability 
during subjective target invisibility we observed in V4 coincided with amplitude modulations of lowfrequency oscillations in the theta, alpha and beta bands, and were highly correlated to activity in the alpha range on a site-by-site basis (Chapter III), suggesting that the two measures are inherently linked. We established equivalent evidence for the hypothesis of neural variability being a reflection of lowfrequency power in the human EEG and showed that alpha amplitudes and trial-to-trial variability closely covaried over time and were strongly correlated across individuals (Chapter IV), supporting recent scientific advances in relating both measures. Based on similar observations that were previously made in the beta frequency range it is likely that this relationship is not exclusive to the alpha band, although alpha oscillations appear to be the largest contribution to neural variability as they constitute the most prominent rhythm of the primate brain. We found both neural variability quenching and ERD in the alpha band to be highly correlated with prestimulus alpha activity in the human EEG, strongly supporting the hypothesis of a dependence of stimulus-induced changes in neural activity on the dynamics of the ongoing activity preceding them as has been proposed by earlier studies. Similarly, we found spontaneous activity prior to stimulus onset to be considerably less variable in the macaque thalamus than in simultaneously recorded extrastriate visual cortex, while neural variability quenching did not occur to the same degree in the thalamus where trial-to-trial variability was already low (Chapter II). The work presented here thus contributes to the current establishment of the relationship between neural variability and alpha oscillations and links stimulus-induced changes in brain activity that have previously been associated with individual perceptual performance to excitability fluctuations present in ongoing activity, suggesting that such dynamics are brought about by mechanisms of anticipatory selective attention. While both spiking variability and low-frequency LFP power during perceptual suppression as well as prestimulus alpha amplitudes differentiated between perceptual outcomes of GFS on otherwise physically identical trials, we propose that these modulations do not reflect visual awareness itself but its attentional prerequisites. 


\subsection{References}

Arazi A, Censor N, Dinstein I (2017a) Neural Variability Quenching Predicts Individual Perceptual Abilities. The Journal of Neuroscience 37:97-109.

Arazi A, Gonen-Yaacovi G, Dinstein I (2017b) The Magnitude of Trial-By-Trial Neural Variability Is Reproducible over Time and across Tasks in Humans. eNeuro 4 Available at: https://www.ncbi.nlm.nih.gov/pmc/articles/PMC5739532/ [Accessed May 11, 2019].

Arazi A, Yeshurun Y, Dinstein I (2019) Neural Variability Is Quenched by Attention. The Journal of Neuroscience 39:5975-5985.

Arieli A, Sterkin A, Grinvald A, Aertsen A (1996) Dynamics of Ongoing Activity: Explanation of the Large Variability in Evoked Cortical Responses. Science 273:1868-1871.

Becker R, Reinacher M, Freyer F, Villringer A, Ritter P (2011) How Ongoing Neuronal Oscillations Account for Evoked fMRI Variability. Journal of Neuroscience 31:11016-11027.

Benwell CSY, Tagliabue CF, Veniero D, Cecere R, Savazzi S, Thut G (2017) Prestimulus EEG Power Predicts Conscious Awareness But Not Objective Visual Performance. eNeuro 4 Available at: https://www.ncbi.nlm.nih.gov/pmc/articles/PMC5732016/ [Accessed April 29, 2019].

Bonneh YS, Cooperman A, Sagi D (2001) Motion-induced blindness in normal observers. Nature 411:798-801.

Bonneh YS, Donner TH, Cooperman A, Heeger DJ, Sagi D (2014) Motion-Induced Blindness and Troxler Fading: Common and Different Mechanisms Lu Z-L, ed. PLoS ONE 9:e92894.

Broday-Dvir R, Grossman S, Furman-Haran E, Malach R (2018) Quenching of spontaneous fluctuations by attention in human visual cortex. Neuroimage 171:84-98.

Carandini M, Anthony Movshon J, Ferster D (1998) Pattern adaptation and cross-orientation interactions in the primary visual cortex. Neuropharmacology 37:501-511.

Carandini M, Ferster D (1997) A Tonic Hyperpolarization Underlying Contrast Adaptation in Cat Visual Cortex. Science 276:949-952.

Carter O, Luedeman R, Mitroff S, Nakayama K (2008) Motion induced blindness: The more you attend the less you see. Journal of Vision 8:237-237.

Churchland MM et al. (2010) Stimulus onset quenches neural variability: a widespread cortical phenomenon. Nat Neurosci 13:369-378.

Churchland MM, Yu BM, Ryu SI, Santhanam G, Shenoy KV (2006) Neural Variability in Premotor Cortex Provides a Signature of Motor Preparation. J Neurosci 26:3697-3712.

Cohen MR, Maunsell JHR (2009) Attention improves performance primarily by reducing interneuronal correlations. Nature Neuroscience 12:1594-1600.

Coslett HB, Saffran E (1991) Simultanagnosia. To see but not two see. Brain 114 ( Pt 4):1523-1545.

Daniel E, Meindertsma T, Arazi A, Donner TH, Dinstein I (2019) The Relationship between Trial-byTrial Variability and Oscillations of Cortical Population Activity. Sci Rep 9:1-11.

Deco G, Hugues E (2012) Neural Network Mechanisms Underlying Stimulus Driven Variability Reduction. PLOS Comput Biol 8:e1002395.

Dijk H van, Schoffelen J-M, Oostenveld R, Jensen O (2008) Prestimulus Oscillatory Activity in the Alpha Band Predicts Visual Discrimination Ability. J Neurosci 28:1816-1823. 
Donner TH, Sagi D, Bonneh YS, Heeger DJ (2008) Opposite neural signatures of motion-induced blindness in human dorsal and ventral visual cortex. J Neurosci 28:10298-10310.

Ergenoglu T, Demiralp T, Bayraktaroglu Z, Ergen M, Beydagi H, Uresin Y (2004) Alpha rhythm of the EEG modulates visual detection performance in humans. Cognitive Brain Research 20:376-383.

Funk AP, Pettigrew JD (2003) Does Interhemispheric Competition Mediate Motion-Induced Blindness? A Transcranial Magnetic Stimulation Study. Perception 32:1328-1338.

Geng H, Song Q, Li Y, Xu S, Zhu Y (2007) Attentional modulation of motion-induced blindness. Chin Sci Bull 52:1063-1070.

Gorea A, Caetta F (2009) Adaptation and prolonged inhibition as a main cause of motion-induced blindness. Journal of Vision 9:16-16.

Goris RLT, Movshon JA, Simoncelli EP (2014) Partitioning neuronal variability. Nat Neurosci 17:858865.

Hanslmayr S, Aslan A, Staudigl T, Klimesch W, Herrmann CS, Bäuml K-H (2007) Prestimulus oscillations predict visual perception performance between and within subjects. Neurolmage $37: 1465-1473$.

Harris KD, Thiele A (2011) Cortical state and attention. Nature Reviews Neuroscience 12:509-523.

He S, Cavanagh P, Intriligator J (1996) Attentional resolution and the locus of visual awareness. Nature 383:334-337.

lemi L, Chaumon M, Crouzet SM, Busch NA (2017) Spontaneous Neural Oscillations Bias Perception by Modulating Baseline Excitability. J Neurosci 37:807-819.

Ikkai A, Dandekar S, Curtis CE (2016) Lateralization in Alpha-Band Oscillations Predicts the Locus and Spatial Distribution of Attention Ahveninen J, ed. PLOS ONE 11:e0154796.

Kelly SP, Lalor EC, Reilly RB, Foxe JJ (2006) Increases in Alpha Oscillatory Power Reflect an Active Retinotopic Mechanism for Distracter Suppression During Sustained Visuospatial Attention. Journal of Neurophysiology 95:3844-3851.

Lamme VAF (2003) Why visual attention and awareness are different. Trends in Cognitive Sciences 7:12-18.

Lange J, Oostenveld R, Fries P (2013) Reduced Occipital Alpha Power Indexes Enhanced Excitability Rather than Improved Visual Perception. J Neurosci 33:3212-3220.

Ling S, Carrasco M (2006) Sustained and transient covert attention enhance the signal via different contrast response functions. Vision Research 46:1210-1220.

Lou L (1999) Selective peripheral fading: evidence for inhibitory sensory effect of attention. Perception 28:519-526.

Maier A, Wilke M, Aura C, Zhu C, Ye FQ, Leopold DA (2008) Divergence of fMRI and neural signals in V1 during perceptual suppression in the awake monkey. Nat Neurosci 11:1193-1200.

McDermott KC, Malkoc G, Mulligan JB, Webster MA (2010) Adaptation and visual salience. Journal of Vision 10:17-17.

Mitchell JF, Sundberg KA, Reynolds JH (2007) Differential attention-dependent response modulation across cell classes in macaque visual area V4. Neuron 55:131-141.

Mitchell JF, Sundberg KA, Reynolds JH (2009) Spatial Attention Decorrelates Intrinsic Activity Fluctuations in Macaque Area V4. Neuron 63:879-888. 
Mo J, Schroeder CE, Ding M (2011) Attentional Modulation of Alpha Oscillations in Macaque Inferotemporal Cortex. J Neurosci 31:878-882.

Oram MW (2011) Visual Stimulation Decorrelates Neuronal Activity. Journal of Neurophysiology 105:942-957.

Parker AJ, Newsome WT (1998) SENSE AND THE SINGLE NEURON: Probing the Physiology of Perception. Annual Review of Neuroscience 21:227-277.

Pfurtscheller G, Lopes da Silva FH (1999) Event-related desynchronization. Handbook of Electroencephalography and Clinical Neurophysiology. Revised Series, Volume 6.

AmsterdamElsevier Science. Available at: https://dare.uva.nl/search?identifier=5dbe2072-6e92-4498b9c2-fb490152715e [Accessed June 17, 2020].

Poland E, Donner TH, Müller K-M, Leopold DA, Wilke M (2019) Thalamus exhibits less sensory variability quenching than cortex. Sci Rep 9:1-12.

Rajan K, Abbott LF, Sompolinsky H (2010) Stimulus-dependent suppression of chaos in recurrent neural networks. Phys Rev E 82:011903.

Ress D, Backus BT, Heeger DJ (2000) Activity in primary visual cortex predicts performance in a visual detection task. Nature Neuroscience 3:940-945.

Rhodes G, Jeffery L, Evangelista E, Ewing L, Peters M, Taylor L (2011) Enhanced attention amplifies face adaptation. Vision Research 51:1811-1819.

Riehle A, Brochier T, Nawrot M, Grün S (2018) Behavioral Context Determines Network State and Variability Dynamics in Monkey Motor Cortex. Front Neural Circuits 12 Available at: https://www.frontiersin.org/articles/10.3389/fncir.2018.00052/full [Accessed June 23, 2020].

Rihs TA, Michel CM, Thut G (2007) Mechanisms of selective inhibition in visual spatial attention are indexed by a-band EEG synchronization. European Journal of Neuroscience 25:603-610.

Rizzo M, Robin DA (1990) Simultanagnosia: A defect of sustained attention yields insights on visual information processing. NEUROLOGIE 40:447-455.

Samaha J, lemi L, Postle BR (2017) Prestimulus alpha-band power biases visual discrimination confidence, but not accuracy. Consciousness and Cognition 54:47-55.

Sauseng P, Klimesch W, Stadler W, Schabus M, Doppelmayr M, Hanslmayr S, Gruber WR, Birbaumer N (2005a) A shift of visual spatial attention is selectively associated with human EEG alpha activity. Eur J Neurosci 22:2917-2926.

Sauseng P, Klimesch W, Stadler W, Schabus M, Doppelmayr M, Hanslmayr S, Gruber WR, Birbaumer N (2005b) A shift of visual spatial attention is selectively associated with human EEG alpha activity. Eur J Neurosci 22:2917-2926.

Schölvinck ML, Rees G (2009) Attentional influences on the dynamics of motion-induced blindness. Journal of Vision 9:38-38.

Schölvinck ML, Saleem AB, Benucci A, Harris KD, Carandini M (2015) Cortical State Determines Global Variability and Correlations in Visual Cortex. J Neurosci 35:170-178.

Schurger A, Sarigiannidis I, Naccache L, Sitt JD, Dehaene S (2015) Cortical activity is more stable when sensory stimuli are consciously perceived. PNAS 112:E2083-E2092.

Shadlen MN, Britten KH, Newsome WT, Movshon JA (1996) A computational analysis of the relationship between neuronal and behavioral responses to visual motion. J Neurosci 16:1486-1510.

Thut G (2006) -Band Electroencephalographic Activity over Occipital Cortex Indexes Visuospatial Attention Bias and Predicts Visual Target Detection. Journal of Neuroscience 26:9494-9502. 
Tong F, Nakayama K, Vaughan JT, Kanwisher N (1998) Binocular Rivalry and Visual Awareness in Human Extrastriate Cortex. Neuron 21:753-759.

Wilke M, Logothetis NK, Leopold DA (2003) Generalized Flash Suppression of Salient Visual Targets. Neuron 39:1043-1052.

Wilke M, Logothetis NK, Leopold DA (2006) Local field potential reflects perceptual suppression in monkey visual cortex. PNAS 103:17507-17512.

Wolfe JM (1984) Reversing ocular dominance and suppression in a single flash. Vision Research 24:471-478.

Worden MS, Foxe JJ, Wang N, Simpson GV (2000) Anticipatory biasing of visuospatial attention indexed by retinotopically specific alpha-band electroencephalography increases over occipital cortex. J Neurosci 20:RC63.

Wöstmann M, Alavash M, Obleser J (2019) Alpha Oscillations in the Human Brain Implement Distractor Suppression Independent of Target Selection. J Neurosci 39:9797-9805.

Yeh T, Lee BB, Kremers J (1996) The time course of adaptation in macaque retinal ganglion cells. Vision Research 36:913-931.

Zohary E, Shadlen MN, Newsome WT (1994) Correlated neuronal discharge rate and its implications for psychophysical performance. Nature 370:140-143. 


\section{Acknowledgements}

Thank you to my supervisor Prof. Dr. Melanie Wilke for providing invaluable guidance throughout these research projects and thank you to Prof. Dr. Stefan Treue and Prof. Dr. Annekathrin Schacht for advising me throughout my doctoral studies.

Special thanks also to Dr. Iris Steinmann and Severin Heumüller as well as all past and present members of the Department of Cognitive Neurology at the University Medical Center Göttingen (UMG), and last but not least my parents, Karin and Manfred Poland, for their continued support. 


\section{Curriculum vitae}

\section{General information}

Name: Eva Poland

Address: Tegeler Weg 12, 37085 Göttingen

Date of birth: 29.01.1988

Place of birth: Nürnberg

Phone: 015128294907

Nationality: German

E-mail: eva.poland@gmx.de

\section{Education}

1994 - 2007 Mathematisch-naturwissenschaftliches Gymnasium Eckental

29.06.2007 Certificate of general university qualification (Abitur), grade 2.1

2007 - 2011 Georg-August-University Göttingen

10.10.2011 Bachelor's degree in Biology, study focus Neuroscience, grade 1.9

B.Sc. thesis at the Department of Cognitive Neuroscience (Prof. Dr. Stefan Treue) - grade 1.0 A comparison of oculomotor behaviour and perceptual judgement in direction discrimination

2011 - 2014 Georg-August-University Göttingen

16.09.2014 Master's degree in Neurobiology, grade 1.5

M.Sc. thesis at the Department of Cognitive Neurology (Prof. Dr. Melanie Wilke) - grade 1.5 Effects of transcranial alternating current stimulation on the perception of time

2014 - 2020 Department of Cognitive Neurology, University Medical Center Göttingen (UMG) 2016 - 2020 Georg-August-University Göttingen, GGNB Systems Neuroscience Ph.D. program

Ph.D. thesis at the Department of Cognitive Neurology (Prof. Dr. Melanie Wilke)

Neural correlates of conscious visual processing 
